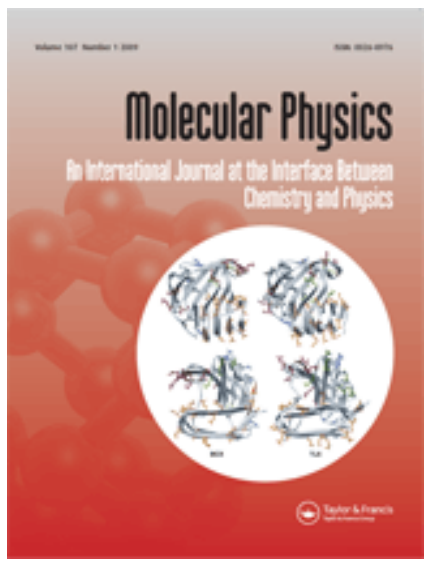

\title{
The accuracy of local MP2 methods for conformational energies
}

\begin{tabular}{|c|c|}
\hline Journal: & Molecular Physics \\
\hline Manuscript ID: & TMPH-2008-0163.R1 \\
\hline Manuscript Type: & Full Paper \\
\hline $\begin{array}{r}\text { Date Submitted by the } \\
\text { Author: }\end{array}$ & 16-Jul-2008 \\
\hline Complete List of Authors: & $\begin{array}{l}\text { Kaminsky, Jakub; Czech Academy of Sciences, Institute of Organic } \\
\text { Chemistry and Biochemistry } \\
\text { Mata, Ricardo; Institut fuer Theoretische Chemie } \\
\text { Werner, Hans-Joachim; Institute for Theoretical Chemistry } \\
\text { Jensen, Frank; University of Aarhus, Department of Chemistry }\end{array}$ \\
\hline Keywords: & LMP2, conformations, peptides \\
\hline \multicolumn{2}{|c|}{$\begin{array}{l}\text { Note: The following files were submitted by the author for peer review, but cannot be converted } \\
\text { to PDF. You must view these files (e.g. movies) online. }\end{array}$} \\
\hline $\begin{array}{l}\text { conformers.tex } \\
\text { conformers.bbl }\end{array}$ & \\
\hline
\end{tabular}

\section{s ScholarONE \\ Manuscript Central}




\title{
The accuracy of local MP2 methods for conformational energies
}

\author{
Jakub Kaminsky \\ Institute of Organic Chemistry and Biochemistry, \\ Czech Academy of Sciences, 16610 Prague, Czech Republic \\ Ricardo A. Mata* and Hans-Joachim Werner \\ Institut für Theoretische Chemie, Universität Stuttgart, \\ Pfaffenwaldring 55, D-70569 Stuttgart, Germany \\ Frank Jensen $^{\dagger}$ \\ Department of Chemistry, University of Aarhus, DK-8000 Aarhus, Denmark
}

\begin{abstract}
The relative energies of 95 conformers of four peptide models are studied using MP2 and LMP2 methods and correlation consistent basis sets ranging from double-zeta to augmented quintuplezeta quality. It is found that both methods yield quite similar results, and the differences between MP2 and LMP2 decrease systematically with increasing basis set. Due to reduced intramolecular basis set superposition effects (BSSE) the LMP2 results converge more slowly to basis set limit for most of these rather small systems. However, for larger peptides, the BSSE has a very large effect on the energy difference between extended and helical structures, leading to a very strong basis set dependence of the canonical MP2 results. It is demonstrated for alanine octapeptides that the basis set error exceeds 30 and $20 \mathrm{~kJ} / \mathrm{mol}$, respectively, if augmented double-zeta and triple-zeta basis sets are used. On the other hand, the LMP2 results are only slightly affected by the basis set size, and even with augmented double-zeta basis sets reasonably accurate results are obtained. Furthermore, for the larger systems the computation times for the LMP2 calculations are shown to be up to one order or magnitude shorter than for canonical MP2 calculations with the same basis set.
\end{abstract}

\footnotetext{
* Present address: Grupo de Física-Matemática da Universidade de Lisboa, Avenue Professor Gama Pinto 2, 1649-003 Lisboa, Portugal

$\dagger$ Corresponding author. Email:frj@chem.au.dk
} 


\section{INTRODUCTION}

Sampling the low-energy region of the phase-space is an essential element for simulating the behavior of macromolecules at finite temperature conditions. The only realistic energy functions for large-scale simulations are of the force field type, but it is desirable to establish the inherent accuracy of these methods. Two of us have recently shown that most force fields in common use have problems reproducing geometries and relative energies of conformations of small peptide models, typically accounting for only half of the conformations. ${ }^{1}$ These conclusions were obtained by generating a reference set of structures and energies by a hierarchy of electronic structure methods, with geometry optimizations at the MP2/aug-ccpVDZ level and relative energies obtained by extrapolating MP2 energies to the basis set limit and adding corrections for higher order electron correlation effects calculated at the $\operatorname{CCSD}(\mathrm{T})$ level. For molecules with localized bonding it is anticipated that relative energies of conformations will be insensitive to electron correlation beyond MP2, and it was found that the MP2/aug-cc-pVTZ level represented a suitable compromise between accuracy and computational cost, producing a typical accuracy of $1 \mathrm{~kJ} / \mathrm{mol}$ for relative energies. We note that Hartree-Fock and density functional methods will be of limited use for systems with several degrees of conformational freedom, as the inadequacy of describing intramolecular dispersion will lead to artifacts in the resulting energy surface.

Having settled on the MP2 method as a suitable level of theory for generating reliable reference data for parameterizing force fields, there remains a question of the computationally most efficient formulation of MP2 theory. The conventional approach is based on canonical molecular orbitals, which leads to a formal $\mathcal{O}\left(\mathcal{N}^{5}\right)$ scaling of computer time with system size. The local MP2 (LMP2) method ${ }^{2-9}$ employs a localization scheme of the molecular orbitals prior to the MP2 step, and by utilizing the locality in the selection of correlating orbitals, the LMP2 method will approach linear scaling for large systems. An additional strategy to reduce the computational requirements is to expand products of functions in an auxiliary basis set, ${ }^{10-12}$ a procedure usually called resolution of identity (RI) or density fitting (DF). Both of these procedures involve additional approximations beyond the regular MP2. In the current work we present extensive benchmark data to investigate the accuracy of these approximations relative to conventional MP2. An earlier study on the use of LMP2 for the calculation of conformational energies can be found in Ref. 13. 


\section{METHOD}

All geometries used in Section III A have been taken as the MP2/aug-cc-pVDZ optimized reported previously, ${ }^{1}$ and the performance for energies relative to the lowest energy conformations was evaluated as the mean and maximum absolute deviations (MAD and MAX) relative to the canonical MP2 results using the cc-pVnZ ${ }^{14}$ and aug-cc-pVn $Z^{15}(n=2-5)$ basis set families. As will be shown below, the additional diffuse functions in the augmented basis sets are essential for achieving rapid basis set convergence at both the Hartree-Fock (HF) and the MP2 levels. These functions are particularly important for the description of the nitrogen and oxygen lone pairs. However, the diffuse functions on the hydrogen atoms have only a rather small effect on the relative energies. Therefore, as a cost effective compromise we recommend mixed basis sets, where the cc-pVnZ sets are used for the hydrogen atoms, and the aug-cc-pVnZ sets for all other atoms. These basis sets will be denoted aug'-cc-pVnZ. For each of the 95 conformers, the MP2 complete basis set limit (CBS) has been estimated using an exponential extrapolation of the HF/aug'-cc-pVnZ energies $(n=3-5)$, and a two-point $n^{-3}$ extrapolation of the MP2/aug'-cc-pVnZ $(n=4-5)$ correlation energies. ${ }^{16}$ These estimates will be denoted MP2/CBS[45].

In Section III B, the initial extended and helical structures of alanine tetra-, hexa- and octapeptide were created in Maestro, the graphic interface program of the MacroModel package. ${ }^{17}$ Three, five and seven aminoacid units, respectively, were used for the construction of our peptides. In addition, acetyl and N-methyl functional groups were added to the Cterminus and N-terminus to mimic the environment in a longer peptide chain. Geometries were first pre-optimized at the MM level with the MMFFs ${ }^{18}$ force field. The structures were then refined using the B-P86 density functional ${ }^{19,20}$ in combination with the TZVP basis set. ${ }^{21}$ Since no extensive search of the PES was made, there is no guarantee that the optimized structures are the global minima. However, for the purpose of the present 
benchmarks this is of no relevance.

Density fitting approximations ${ }^{11,12}$ have been employed in the $\mathrm{HF}$ as well as the MP2/LMP2 steps, using, respectively, the cc-pVnZ/JKFIT and cc-pVnZ/MP2FIT or augcc-pVnZ/MP2FIT auxiliary basis sets of Weigend et al. ${ }^{22,23}$ In each case $n$ corresponds to the orbital basis. Local density fitting approximations ${ }^{11,12}$ were disabled, except when otherwise noted. All calculations have been carried out using the MOLPRO ${ }^{24}$ program package.

\section{RESULTS AND DISCUSSION}

\section{A. Small peptide model systems}

By comparison of the MP2 and DF-MP2 results it was found that typical errors due to the density fitting approximation are $\sim 0.01 \mathrm{~kJ} / \mathrm{mol}$. The maximum deviations never exceeded $0.1 \mathrm{~kJ} / \mathrm{mol}$ and are therefore well within the $1 \mathrm{~kJ} / \mathrm{mol}$ target accuracy. Thus, in agreement with earlier investigations on conformational energies ${ }^{25}$ and other properties, the errors introduced by density fitting are negligible. Therefore, density fitting was used in all further calculations. For simplicity, the prefix DF will be omitted in the following.

The LMP2 method involves two distinct approximations: first, the excitations are restricted to pair-specific domains of projected atomic orbitals (PAOs). Secondly, distant pairs can be treated by multipole approximations ${ }^{7,8,26}$ or neglected. Both approximations together lead to linear scaling of the computational cost with molecular size. ${ }^{7,8,11}$ In this benchmark set, however, the systems were rather small and all pairs have always been included, i.e., distant pair approximations were not applied. The impact of pair approximations will be discussed for larger systems in Section III B.

When computing small energy differences using local methods it is essential to make sure that consistent domains are employed for all conformers. In most previous local correlation calculations a method proposed by Boughton and Pulay $^{27}(\mathrm{BP})$ has been used to determine the domains automatically. Unfortunately, the domains generated by this procedure are sensitive to the basis set and may also change as a function of the geometry. The former problem may lead to an erratic basis set convergence, the latter to small steps on potential energy surfaces ${ }^{28-30}$ and to unbalanced results. ${ }^{30}$ This also happens when considering different conformers, and we found that in the present benchmark the BP method leads 
to quite unsatisfactory results, with maximum errors of up to about $10 \mathrm{~kJ} / \mathrm{mol}$ relative to canonical MP2. Since the geometry changes between different conformers are substantial, the problem cannot be avoided by simply freezing the domains. We also tested the LMP2 implementation of the JAGUAR program ${ }^{31}$ for the cc-pVDZ and cc-pVTZ basis sets, and in these cases the average and maximum errors were even somewhat larger than with the BP procedure in MOLPRO.

Recently, two of us have developed an alternative method $^{32}$ to determine the domains. This procedure is based on the natural population analysis (NPA) and natural localized molecular orbitals (NLMOs) proposed originally by Reed and Weinhold. ${ }^{33,34}$ It yields domains that are much more stable with respect to the basis set and geometry than the BP method. In the present work we have applied this NLMO/NPA method in LMP2 calculations for all 95 conformers using 10 different basis sets ranging from cc-pVDZ to aug'-cc-pV5Z. The NPA domain selection criterion $^{32}$ was chosen to be 0.03 . For each of the four model peptides the domains were consistent (i.e. the same atoms were selected for each orbital domain) for all conformers and 10 different basis sets. This confirms the superior stability of the new NLMO/NPA method.

The results are summarized in Table I, which shows the mean and maximum absolute deviations of the LMP2 and MP2 results for each basis set relative to the MP2/CBS[45] results. In addition, in the last two columns the mean and maximum absolute deviations between the LMP2 and MP2 results for each basis set are presented. The geometries and individual energies can be found in the supplementary material. The canonical MP2 method with an aug-cc-pVTZ basis is capable of reproducing the basis set limiting values within $\approx 1$ $\mathrm{kJ} / \mathrm{mol}$. For the aug'-cc-pVnZ basis sets, in which the diffuse functions on the hydrogen atoms are omitted, the errors for MP2 are larger, while for LMP2 they perform almost as well as the full sets. On the other hand, the errors of both MP2 and LMP2 increase significantly if the diffuse functions are entirely omitted, at least for the smaller basis sets. These findings can be rationalized by the different contribution of basis set superposition effects (BSSE), as discussed below. It should be noted that the results for the double-zeta basis sets are rather strongly affected by errors of the HF contribution, and these basis sets are clearly too small for obtaining reliable energy differences.

The LMP2 results display convergence towards nearly the same limiting values as the canonical MP2 method. For the aug'-cc-pVnZ basis sets, the absolute deviations of the 
LMP2 results from the MP2/CBS[45] reference values are typically twice as large as those of the canonical MP2. Without diffuse functions, the differences between MP2 and LMP2 results are even larger. The different convergence of the two methods with increasing basis set is attributed to the BSSE, which will be discussed below.

A closer inspection of the results for the two glycine conformers (Fig. 1) is presented in Fig. 2. The upper panel shows the convergence of the Hartree-Fock energy differences for the cc-pVnZ and aug'-cc-pVnZ basis sets. It is obvious that the inclusion of diffuse functions is essential to achieve rapid convergence. With the aug'-cc-pVDZ basis sets the error still amounts to $1.4 \mathrm{~kJ} / \mathrm{mol}$, but for all larger aug'-cc-pVnZ sets the HF values are very close to the estimated basis set limit. Note that in the present case the HF energy differences are negative, i.e., the energetic order of the two conformers is opposite than for MP2.

The lower panel of Fig. 2 shows the MP2 and LMP2 correlation energy contributions to the energy difference of the two conformers. The energetically lower conformer has a folded structure which is stabilized by hydrogen bonding, while the higher one has a stretched structure. At the more compact folded structure the dynamical correlation energy is larger, and therefore the correlation contribution to the energy difference is positive. This effect should increase with increasing basis set. However, as can be seen in the figure, the MP2 correlation contribution decreases with increasing cardinal number $n$. This can be attributed to the BSSE, which should decrease with $n$. Apparently, in the present case the decrease of the BSSE overcompensates the increase of the correlation contribution, leading overall to a decrease of the energy difference.

The behavior is entirely different for the LMP2 method, in which the BSSE is strongly reduced. ${ }^{6,35,36}$ When standard NPA domains are used, the correlation contribution converges from below to the basis set limit, as one would anticipate in the absence of BSSE. The convergence is slower than for MP2, because there is no compensation of the incomplete basis set error by the BSSE. If the domains are extended by including the PAOs at the first shell of neighboring atoms (LMP2(ext)), part of the BSSE is re-introduced, and in this case the basis set convergence dependence is remarkably weak. The total (HF + LMP2) triple-zeta value differs only by $0.01 \mathrm{~kJ} / \mathrm{mol}$ from the quintuple-zeta one. However, the quintuple-zeta result is still $0.2 \mathrm{~kJ} / \mathrm{mol}$ below the MP2/CBS[45] limit. This may be due to the two missing classes of ionic excitations, which are excluded in the LMP2 ansatz by construction. ${ }^{36}$ Interestingly, the extrapolated LMP2(ext) result differs by less than 0.05 
$\mathrm{kJ} / \mathrm{mol}$ from the MP2 one. However, the extrapolation of local correlation energies should be considered with care, since the domains become more complete with increasing cardinal number, and this may lead to a different asymptotic basis set convergence than in the canonical case. Furthermore, it is of course essential that the domains are consistent for all basis sets. In the present applications, this is the case with the NLMO/NPA method, but not with the BP procedure.

The glycine case discussed above demonstrates well the different basis set convergence of MP2 and LMP2, but it is somewhat special in the sense that the MP2 correlation contribution converges from above to the basis set limit. In most other cases of the present benchmark set the MP2 correlation contributions converge from below to the basis set limit (still being positive if the energetically lowest conformer is taken as the reference), indicating that the BSSE is less dominant than in the glycine case. But the BSSE still improves the MP2 results, in particular for the small basis sets, and therefore the convergence of the LMP2 results is slower. This effect is reduced when extended domains are used: For LMP2(ext)/aug'-ccpVTZ the average and maximum absolute deviations from the MP2/CBS[45] limits are 1.41 and $2.77 \mathrm{~kJ} / \mathrm{mol}$, respectively. These values are only $0.4-0.5 \mathrm{~kJ} / \mathrm{mol}$ larger than the corresponding canonical MP2 values.

\section{B. Alanine peptides}

In order to demonstrate the accuracy and computational savings of the LMP2 method for larger peptides, alanine tetra-, hexa- and octapeptides were chosen as model systems. For each of these peptides, an extended and an $\alpha$-helix conformer were optimized using density functional theory, as described in section III A. The optimized alpha-helix structures are shown in Fig. 3. At these structures, the HF, MP2 and LMP2 conformational energies were computed using aug'-cc-pVnZ basis sets $(n=2-4)$. The MP2/CBS[34] basis set limits were estimated by extrapolation of the triple-zeta and quadruple-zeta correlation energies. In these calculations the HF energies were not extrapolated, and the aug'-cc-pVQZ values were taken as the reference. The results are summarized in Table II.

As for the smaller model systems, the MP2 and LMP2 results converge to approximately the same basis set limits. However, the basis set convergence of the two methods is very different. The most striking result in Table II is the slow basis set convergence of the MP2 
results for the octapeptide folding energy. The MP2/aug'-cc-pVTZ result has a deviation of $20 \mathrm{~kJ} / \mathrm{mol}$ relative to the CBS[34] reference. This amounts to $31 \%$ of the total folding energy. On the other hand, the LMP2 results show a remarkably low basis set dependence. For the aug'-cc-pVDZ basis set this is partly due to error compensation of the HF and correlation contributions, but nevertheless for all three peptides the LMP2/aug'-cc-pVDZ results are already very close to the aug'-cc-pVQZ ones. Very likely, the strong basis set dependence of the canonical MP2 results is mainly due to BSSE effects, which over-stabilize the folded structure. The BSSE is largely eliminated in the LMP2, and this leads to the much better basis set stability, very similar to the case shown in Fig. 1 for the glycine conformers. The same effect was observed by Head-Gordon and coworkers, while investigating the stability of 27 alanine tetrapetides. ${ }^{25}$

The effect is most pronounced for the octapeptide, since the smaller structures, after optimization, tend to distort significantly from the ideal helix conformation, as shown in Fig. 3. In the hexamer, only half of the chain is folded, the other section is extended. In the case of the tetrapeptide, the polypeptide chain is too small and the backbone is approximately planar. However, even in this case, the LMP2 results are almost independent of the basis set, while the MP2 ones change by more than $40 \%$ from aug'-cc-pVDZ to the CBS[34] limit. Although these examples are simplified models, the present results confirm earlier findings made for the alanine tetrapeptide. ${ }^{25}$ Large basis sets are needed when computing folding energies with canonical correlation methods, since BSSE starts to play a crucial role in larger peptides. As the chain size increases, the errors accumulate, and at least quadruple-zeta quality basis set are necessary in order to obtain reasonably accurate results with MP2. ${ }^{37}$ On the other hand, local correlation methods allow to use much smaller basis sets; moreover, for a given basis set the computational effort is drastically reduced, as will be demonstrated in the following.

The computation times of MP2 and LMP2 calculations for the octapeptides are shown in Table III. The savings made by the local approximations are quite dramatic. As compared to the canonical calculation, the total computational time for the extended structure is reduced by one order of magnitude. As expected, the LMP2 calculations for the $\alpha$-helix conformer with a more more three-dimensional structure are slower than for the extended peptide, especially for the iterations, but the savings are still significant. The longer computation times for the helix structure are mainly caused by the larger number of pairs included in 
the calculation (see below).

Further savings are possible through the use of local density fitting approximations, which asymptotically lead to linear scaling of the computational cost with system size. ${ }^{11}$ In the local density fitting method, the 4-index exchange integrals $K_{r s}^{i j}=(r i \mid s j)$ are decomposed into sums of products of 2- and 3-index integrals

$$
\begin{aligned}
K_{r s}^{i j} \approx & \sum_{A \in[i]_{\mathrm{fit}}} D_{r i}^{A}(s j \mid A)+\sum_{B \in[j]_{\mathrm{fit}}}(r i \mid B) D_{s j}^{B} \\
& -\sum_{A \in[i]_{\mathrm{fit}}} \sum_{B \in[j]_{\mathrm{fit}}} D_{r i}^{A} J_{A B} D_{s j}^{B}
\end{aligned}
$$

where the indices $\{A, B\}$ refer to the auxiliary basis set and $D_{r i}^{A}$ are the fitting coefficients obtained by solving systems of linear equations

$$
(r i \mid A)=\sum_{B \in[i]_{\mathrm{fit}}} J_{A B} D_{r i}^{B}
$$

(for further details see Ref. 11). Note that this robust fitting approximation is slightly different than the less symmetric approximation described in Ref. 11.

In a conventional implementation, the summations in eqs. (1) and (2) would run over the full fitting basis, leading to $\mathcal{O}\left(\mathcal{N}^{5}\right)$ scaling. The last two terms in eq. (1) then cancel. In a local calculation, it is possible to use for each orbital pair $i j$ a truncated fitting basis, in which only the auxiliary function in the vicinity of the localized orbitals $i$ and $j$ are included. The default in MOLPRO is to include in the domain $[i]_{\text {fit }}$ the fitting functions at all atoms that are separated from the atoms in the orbital domain $[i]$ by at most 3 bonds $\left(l_{f}=3\right)$. Using this value the effect of the local density fitting approximation on the relative energies is negligible. In fact, in the current case even smaller fitting domains with only the atoms that are separated by at most one bond $\left(l_{f}=1\right)$ from the atoms in the domain $[i]$ are sufficient to obtain accurate results (cf. Table III).

The number of pairs included in the LMP2 and therefore onset of linear scaling and the CPU-time depends on the the distance criterium $R_{v d}$ for neglecting very distant pairs. ${ }^{6,7}$ Orbital pairs $i j$ are neglected if the closest distance $R_{i j}$ between an atom in the orbital domain $[i]$ and an atom in the orbital domain $[j]$ is larger than $R_{v d}$. Reducing this distance criterion below its default value of 15 bohr leads to significant savings, but increases the error in the energy difference (cf. Table III). If the distance criterion is set to 12 bohr the errors already exceed $1 \mathrm{~kJ} / \mathrm{mol}$. The sensitivity of the energy difference is due to the 
different three-dimensional arrangement of the conformers. In the helix structure, more pairs are removed when $R_{v d}$ is decreased: if $R_{v d}$ is reduced from 15 to 12 bohr, about 800 pairs are removed in the helix case, while in the extended conformer only 500 pairs are removed. Therefore, the helix energy is more affected than the energy of the extended conformer, and the energy difference decreases.

Optionally, the integrals for distant pairs $\left(R_{d}<R_{i j} \leq R_{v d}\right)$ can be approximated by multipole approximations, which also reduce the overall cost. ${ }^{8,26}$ However, as shown in Table III for various choices of $R_{d}$, this approximation leads to rather large errors. As already pointed out in Ref. 26, this is due to the neglect of certain exchange contributions, and such approximations are therefore not recommended in the study of intermolecular forces or for cases in which long-range interactions play a crucial role.

Finally, it should be noted that the CPU-time for the Hartree-Fock calculation (about 8.3 hours, using density fitting and the aug'-cc-pVTZ basis) exceeds that of the LMP2 step. The differences are even greater when the aug'-cc-pVQZ basis (3860 contracted functions) is used. The HF and MP2 calculations took about 85 and 110 hours, respectively, while the LMP2 calculation took only 28 hours (helix structure, without local density fitting approximations).

\section{CONCLUSIONS}

We have investigated the effect of local approximations on the relative energies of different conformers. In order to obtain reliable LMP2 energy differences it is important that the orbital domains of the different conformers are defined consistently. This can be achieved using a recently proposed method that is based on natural population analysis. ${ }^{32}$ With this method consistent domains were obtained for all conformers and all basis sets studied in this work, and a smooth convergence with increasing basis set size to the basis set limit was always achieved.

For all model systems studied, the MP2 and LMP2 methods yield very similar results at the basis set limit. However, due to the reduced BSSE in the LMP2, the convergence of MP2 and LMP2 with increasing basis sets is different. For most of the small systems in the benchmark set the convergence of the LMP2 results is found to be somewhat slower than for MP2, since in these systems the BSSE partly compensates for other basis set deficiencies. 
1 J. Kaminsky and F. Jensen, J. Chem. Theo. Comp. 3, 1774 (2007).

2 P. Pulay, Chem. Phys. Lett. 100, 151 (1983).

3 S. Saebø and P. Pulay, Chem. Phys. Lett. 113, 13 (1985).

4 P. Pulay and S. Saebø, Theor. Chim. Acta 69, 357 (1986).

5 S. Saebø and P. Pulay, J. Chem. Phys. 86, 914 (1987).

6 C. Hampel and H.-J. Werner, J. Chem. Phys. 104, 6286 (1996).

7 M. Schütz, G. Hetzer, and H.-J. Werner, J. Chem. Phys. 111, 5691 (1999).

8 G. Hetzer, M. Schütz, H. Stoll, and H.-J. Werner, J. Chem. Phys. 113, 9443 (2000).

9 S. Saebo and P. Pulay, J. Chem. Phys. 115, 3975 (2001).

10 O. Vahtras, J. Almlöf, and M. W. Feyereisen, Chem. Phys. Lett. 213, 514 (1993).

11 H.-J. Werner, F. R. Manby, and P. Knowles, J. Chem. Phys. 118, 8149 (2003).

12 R. Polly, H.-J. Werner, F. R. Manby, and P. J. Knowles, Mol. Phys. 102, 2311 (2004).

13 M. D. Beachy, D. Chasman, R. B. Murphy, T. A. Halgren, and R. A. Friesner, J. Am. Chem. Soc. 119, 5908 (1997).

14 T. H. Dunning, Jr., J. Chem. Phys. 90, 1007 (1989).

15 R. A. Kendall, T. H. Dunning, and R. J. Harrison, J. Chem. Phys. 96, 6796 (1992).

16 K. L. Bak, P. Jørgensen, J. Olsen, T. Helgaker, and W. Klopper, J. Chem. Phys. 112, 9229 (2000). 
17 L. Schrödinger, Macromodel, version 1.9 (2005).

18 T. A. Halgren, J. Comput. Chem. 17, 490 (1996).

19 A. D. Becke, Phys. Rev. A 38, 3098 (1988).

20 J. P. Perdew, Phys. Rev. B 33, 8822 (1986).

21 A. Schäfer, C. Huber, and R. Ahlrichs, J. Chem. Phys. 100, 5829 (1994).

22 F. Weigend, Phys. Chem. Chem. Phys. 4, 4285 (2002).

23 F. Weigend, A. Köhn, and C. Hättig, J. Chem. Phys. 116, 3175 (2002).

24 H.-J. Werner, P. J. Knowles, R. Lindh, F. R. Manby, M. Schütz, et al., Molpro, version 2006.4, a package of ab initio programs (2007), see http://www.molpro.net.

25 R. A. DiStasio Jr., Y. Jung, and M. Head-Gordon, J. Chem. Theory Comput. 1, 862 (2005).

26 G. Hetzer, P. Pulay, and H.-J. Werner, Chem. Phys. Lett. 290, 143 (1998).

27 J. W. Boughton and P. Pulay, J. Comput. Chem. 14, 736 (1993).

28 N. J. Russ and T. D. Crawford, J. Chem. Phys. 121, 691 (2004).

29 J. Subotnik and M. Head-Gordon, J. Chem. Phys. 123, 064108 (2005).

30 R. A. Mata and H.-J. Werner, J. Chem. Phys. 125, 184110 (2006).

31 Jaguar, version 7.0, Schrödinger LLC, New York (2007).

32 R. A. Mata and H.-J. Werner, Mol. Phys. 105, 2753 (2007).

33 A. E. Reed, R. B. Weinstock, and F. Weinhold, J. Chem. Phys. 83, 735 (1985).

34 A. E. Reed and F. Weinhold, J. Chem. Phys. 83, 1736 (1985).

35 S. Saebø, W. Tong, and P. Pulay, J. Chem. Phys. 98, 2170 (1993).

36 M. Schütz, G. Rauhut, and H.-J. Werner, J. Phys. Chem. A 102, 5997 (1998).

37 R. A. DiStasio Jr., R. P. Steele, Y. M. Rhee, Y. Shao, and M. Head-Gordon, J. Comput. Chem. 28, 839 (2007). 
Tables

5

6

7

8

9

10

11

12

13

14

15

16

17

18

19

20

21

22

23

24

25

26

27

28

29

30

31

32

33

34

35

36

37

38

39

40

41

42

43

44

45

46

47

48

49

50

51

52

53

54

55

56

57

58

59

60 
TABLE I: Mean (MAD) and maximum (MAX) absolute deviations of relative energies (in $\mathrm{kJ} / \mathrm{mol}$ ) for 91 conformations of four model peptide systems.

\begin{tabular}{|c|c|c|c|c|}
\hline \multirow[t]{2}{*}{ Basis set } & \multicolumn{2}{|c|}{$\mathrm{MP}^{a}$} & $\mathrm{LMP}^{a}{ }^{a}$ & $\mathrm{LMP}^{b}$ \\
\hline & MAD & MAX & MAD MAX & MAD MAX \\
\hline cc-pVDZ & 3.02 & 10.55 & 2.347 .21 & 2.396 .02 \\
\hline cc-pVTZ & 0.91 & 3.48 & 1.944 .43 & 2.205 .36 \\
\hline cc-pVQZ & 0.34 & 0.86 & 1.232 .78 & 1.163 .02 \\
\hline cc-pV5Z & 0.20 & 0.47 & 0.822 .00 & 0.671 .71 \\
\hline aug-cc-pVDZ & 1.68 & 4.30 & 3.035 .58 & 1.995 .14 \\
\hline aug-cc-pVTZ & 0.64 & 1.64 & 1.773 .48 & 1.383 .36 \\
\hline aug'-cc-pVDZ $^{c}$ & 2.13 & 5.48 & 3.005 .51 & 1.504 .02 \\
\hline aug'-cc-pVTZ ${ }^{c}$ & 0.92 & 2.38 & 1.853 .61 & 1.102 .79 \\
\hline aug'-cc-pVQZ $^{c}$ & 0.38 & 0.89 & 0.851 .80 & 0.541 .30 \\
\hline aug'-cc-pV5Zc & 0.19 & 0.45 & 0.491 .06 & 0.330 .79 \\
\hline $\mathrm{CBS}[45]^{d}$ & $(0.0)$ & $(0.0)$ & 0.120 .33 & 0.120 .33 \\
\hline
\end{tabular}

a) Relative to the MP2/CBS[45] values.

b) Relative to MP2 values for each basis set.

c) aug' indicates that diffuse functions are included only on non-hydrogen atoms.

d) Basis set limits estimated from extrapolations of the aug'-cc-pVnZ energies, see text. 
TABLE II: Energy difference $E_{\alpha-\text { helix }}-E_{\text {extended }}$ (in $\mathrm{kJ} / \mathrm{mol}$ ) between the helical and extended conformers of the alanine peptides. The HF contribution is included in the MP2 and LMP2 values.

\begin{tabular}{|c|c|c|c|c|}
\hline Peptide & Basis & $\mathrm{HF}$ & MP2 & LMP2 \\
\hline \multirow[t]{4}{*}{$(\text { ala })_{4}$} & aug'-cc-pVDZ & 27.69 & 12.58 & 18.21 \\
\hline & aug'-cc-pVTZ & 29.71 & 15.74 & 18.61 \\
\hline & aug'-cc-pVQZ & 30.71 & 17.26 & 19.06 \\
\hline & $\mathrm{CBS}[34]^{a}$ & - & 18.03 & 19.06 \\
\hline \multirow[t]{4}{*}{$(\text { ala })_{6}$} & aug'-cc-pVDZ & 42.84 & 47.29 & 46.15 \\
\hline & aug'-cc-pVTZ & 41.47 & 45.55 & 44.27 \\
\hline & aug'-cc-pVQZ & 41.61 & 45.62 & 45.18 \\
\hline & $\mathrm{CBS}[34]^{a}$ & - & 45.57 & 45.75 \\
\hline \multirow[t]{4}{*}{$(\text { ala })_{8}$} & aug'-cc-pVDZ & -15.32 & -94.49 & -60.08 \\
\hline & aug'-cc-pVTZ & -2.38 & -84.03 & -63.19 \\
\hline & aug'-cc-pVQZ & -0.87 & -71.78 & -60.84 \\
\hline & $\mathrm{CBS}[34]^{a}$ & - & -63.95 & -60.22 \\
\hline
\end{tabular}

a) Calculated from the aug'-cc-pVTZ and aug'-cc-pVQZ correlation energies and the aug'-cc-pVQZ HF value. 
TABLE III: CPU-times (in minutes, using $2.8 \mathbf{G H z}$ opteron processors) for conventional and local correlation calculations $^{a}$ with the aug'-cc-pVTZ basis set on the extended/helix conformers of the alanine octapeptide. The conformational energies (in $\mathrm{kJ} / \mathrm{mol}) \Delta \mathrm{E}=\mathrm{E}(\alpha$-helix)-E(extended) are also shown.

\begin{tabular}{lccccccc}
\hline \multicolumn{2}{l}{ Method $l_{f}$} & $R_{v d}$ & $R_{d}$ Integrals $^{b}$ & Iterations $^{c}$ & Total & $\Delta \mathrm{E}$ \\
\hline MP2 & - & - & - & - & - & $1509 / 1514$ & -84.03 \\
LMP2 & - & - & - & $162 / 176$ & $413 / 413$ & $590 / 604$ & -63.01 \\
LMP2 & - & 15 & - & $106 / 149$ & $95 / 283$ & $214 / 448$ & -63.19 \\
LMP2 & 1 & 15 & - & $45 / 84$ & $95 / 283$ & $152 / 380$ & -63.13 \\
LMP2 & 1 & 12 & - & $44 / 78$ & $68 / 219$ & $124 / 310$ & -61.91 \\
LMP2 & 1 & 10 & - & $39 / 65$ & $43 / 135$ & $94 / 212$ & -58.19 \\
LMP2 & 1 & 15 & 12 & $44 / 75$ & $94 / 256$ & $152 / 347$ & -62.30 \\
LMP2 & 1 & 15 & 10 & $37 / 70$ & $78 / 242$ & $128 / 328$ & -60.40 \\
LMP2 & 1 & 15 & 8 & $32 / 52$ & $70 / 198$ & $114 / 264$ & -52.48 \\
\hline \hline
\end{tabular}

a) $R_{v d}$ and $R_{d}$ (in bohr) are the distance criteria for neglecting very distant pairs and for using multipole approximations, respectively; $l_{f}$ determines the size of the local fitting domains, see text.

b) CPU-time for integral evaluation and transformation.

c) CPU-time to solve the first-order LMP2 equations. 


\section{Figure Captions}

Figure 1: Ball-and-stick representation of the two glycine tripeptide conformers discussed in the text and Fig. 2. The upper structure corresponds to the minimum energy conformation.

Figure 2: Hartree-Fock (upper panel) and correlation contributions (lower panel) to the relative energy of the glycine conformers as a function of cardinal number $n$ of the basis set.

Figure 3: Ball-and-stick representation of the alanine tetra-, hexa- and octapeptide optimized helical conformers. 
Figures 
FIG. 1: Jakub et al., Molecular Physics
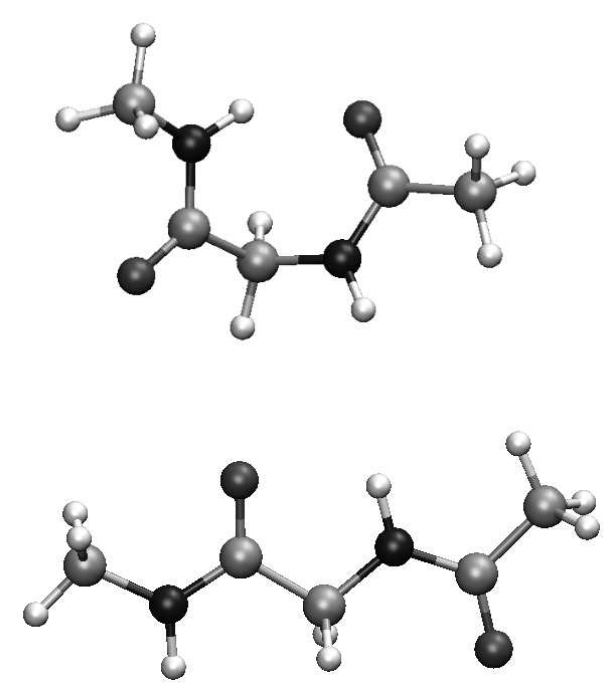
FIG. 2: Jakub et al., Molecular Physics
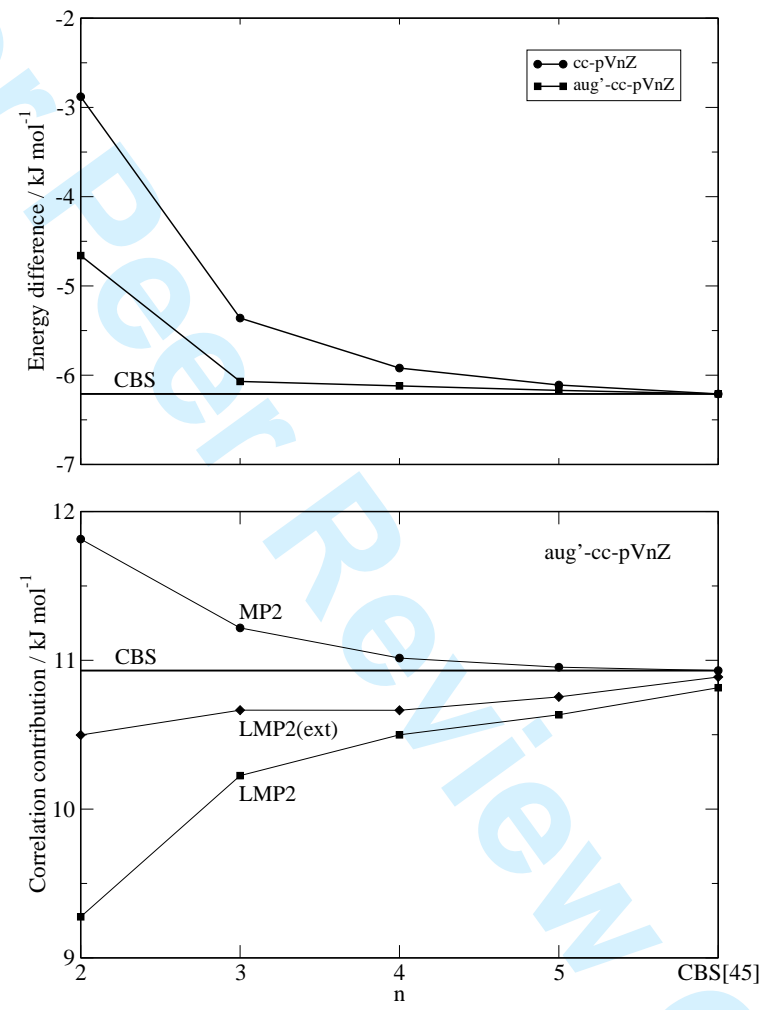
FIG. 3: Jakub et al., Molecular Physics
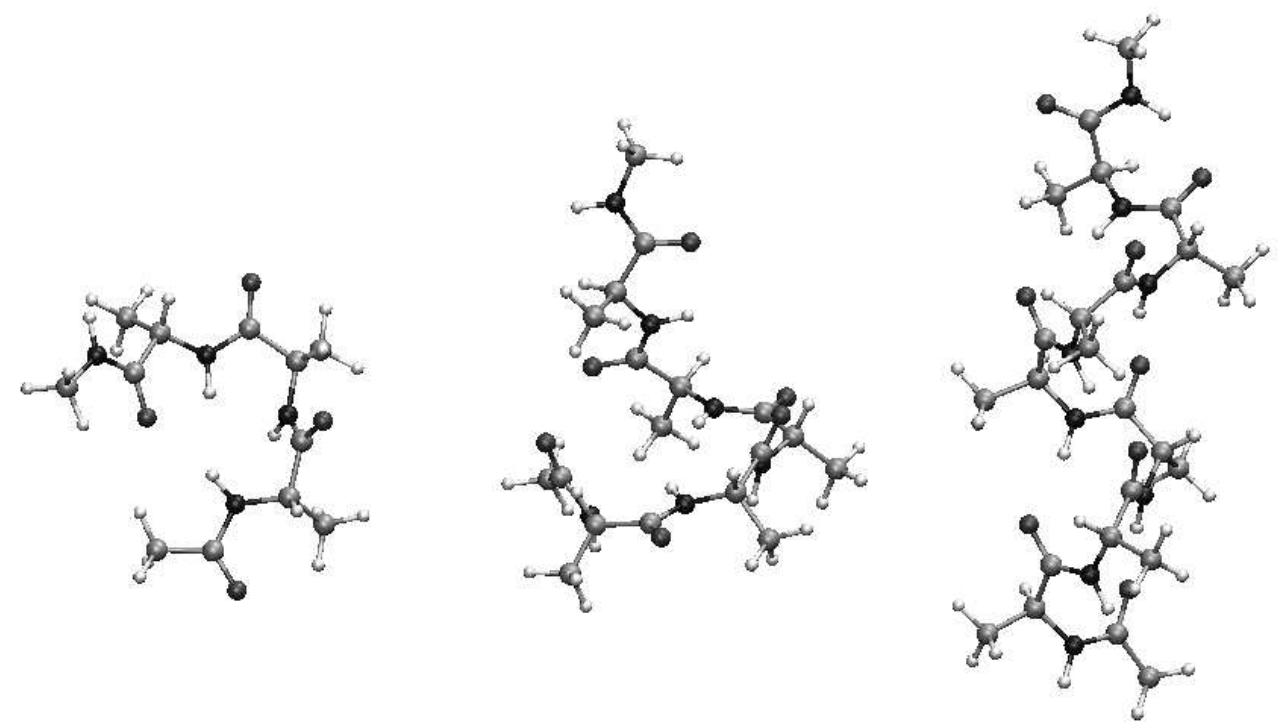

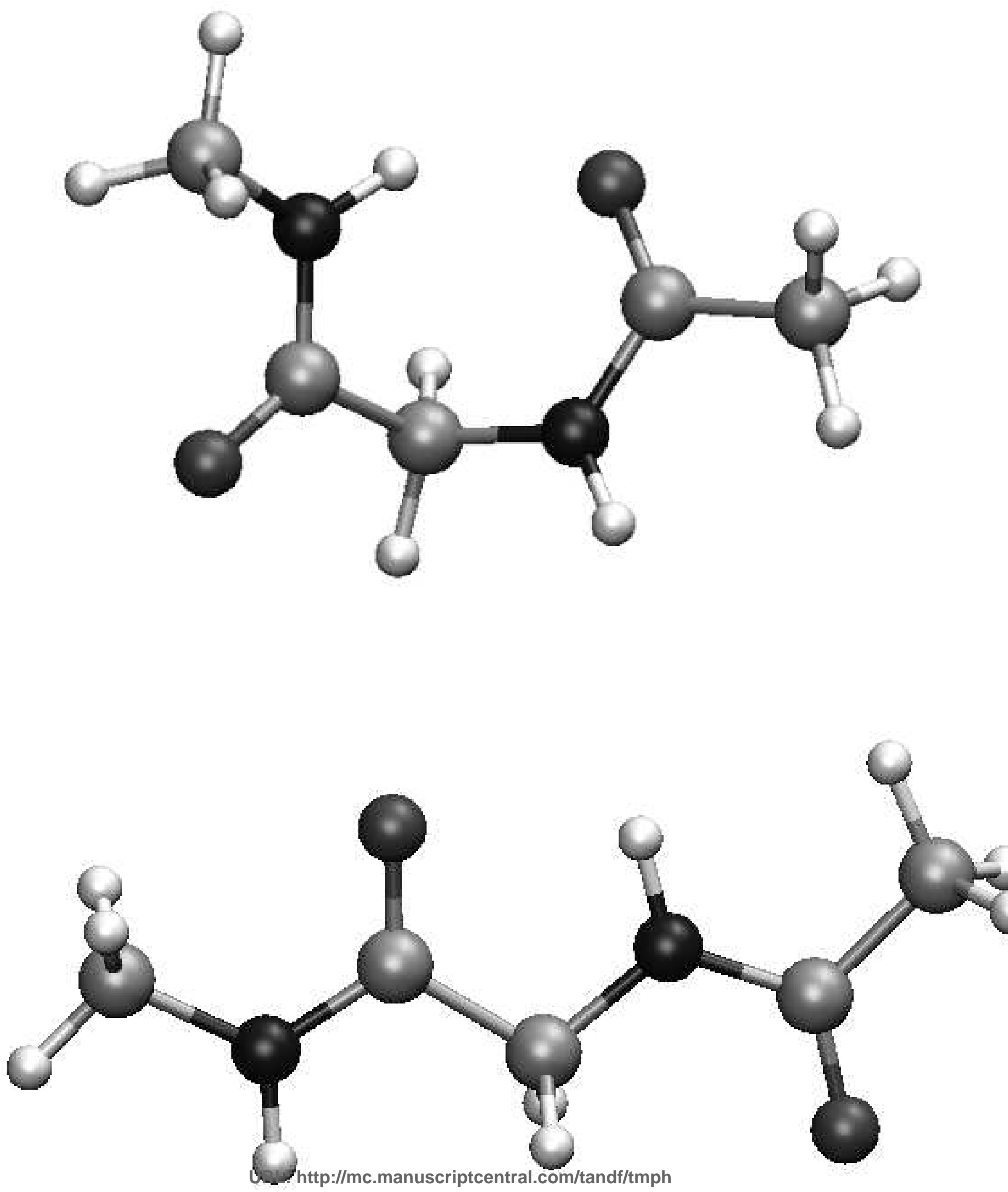


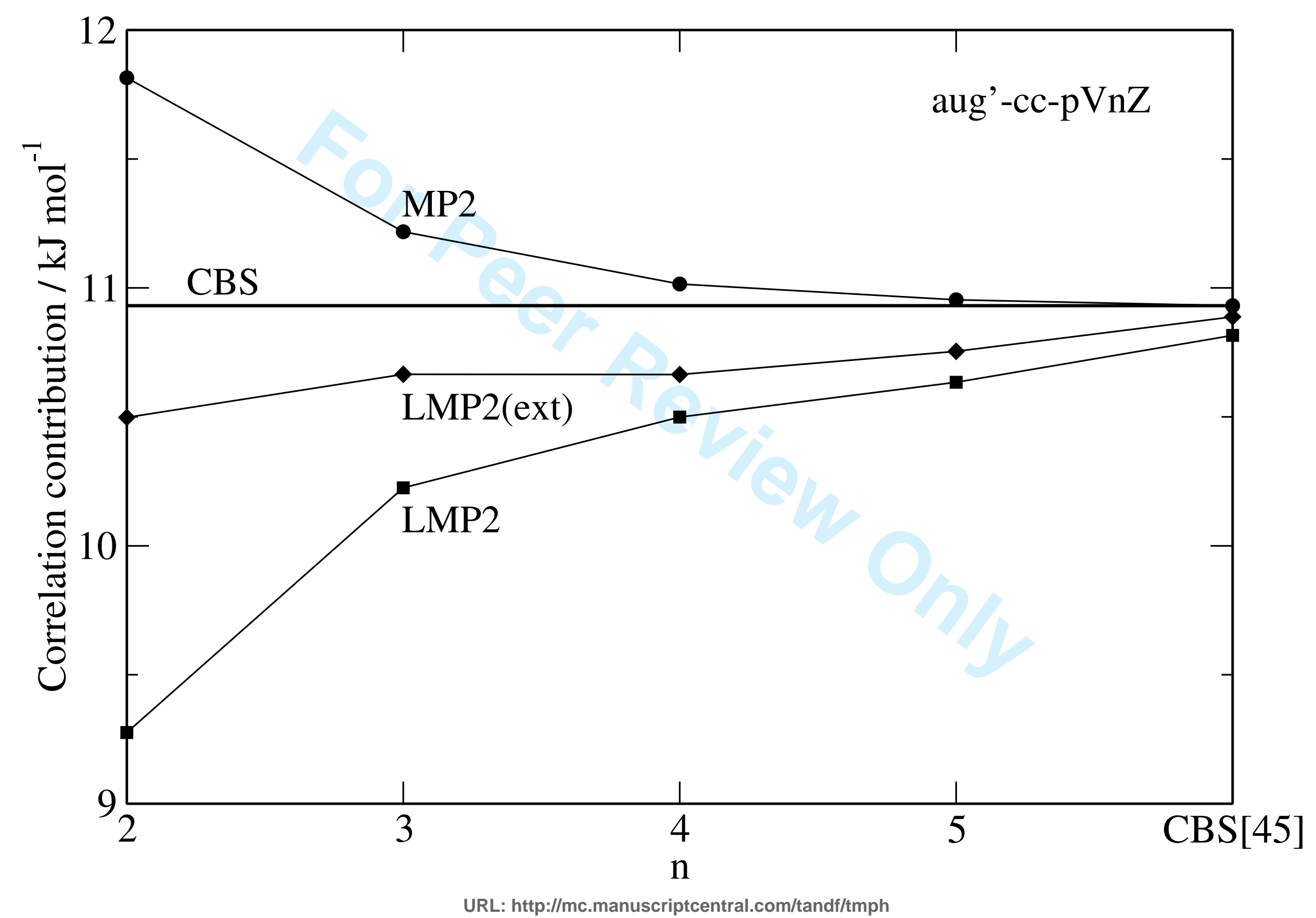

URL: http://mc.manuscriptcentral.com/tandf/tmph 


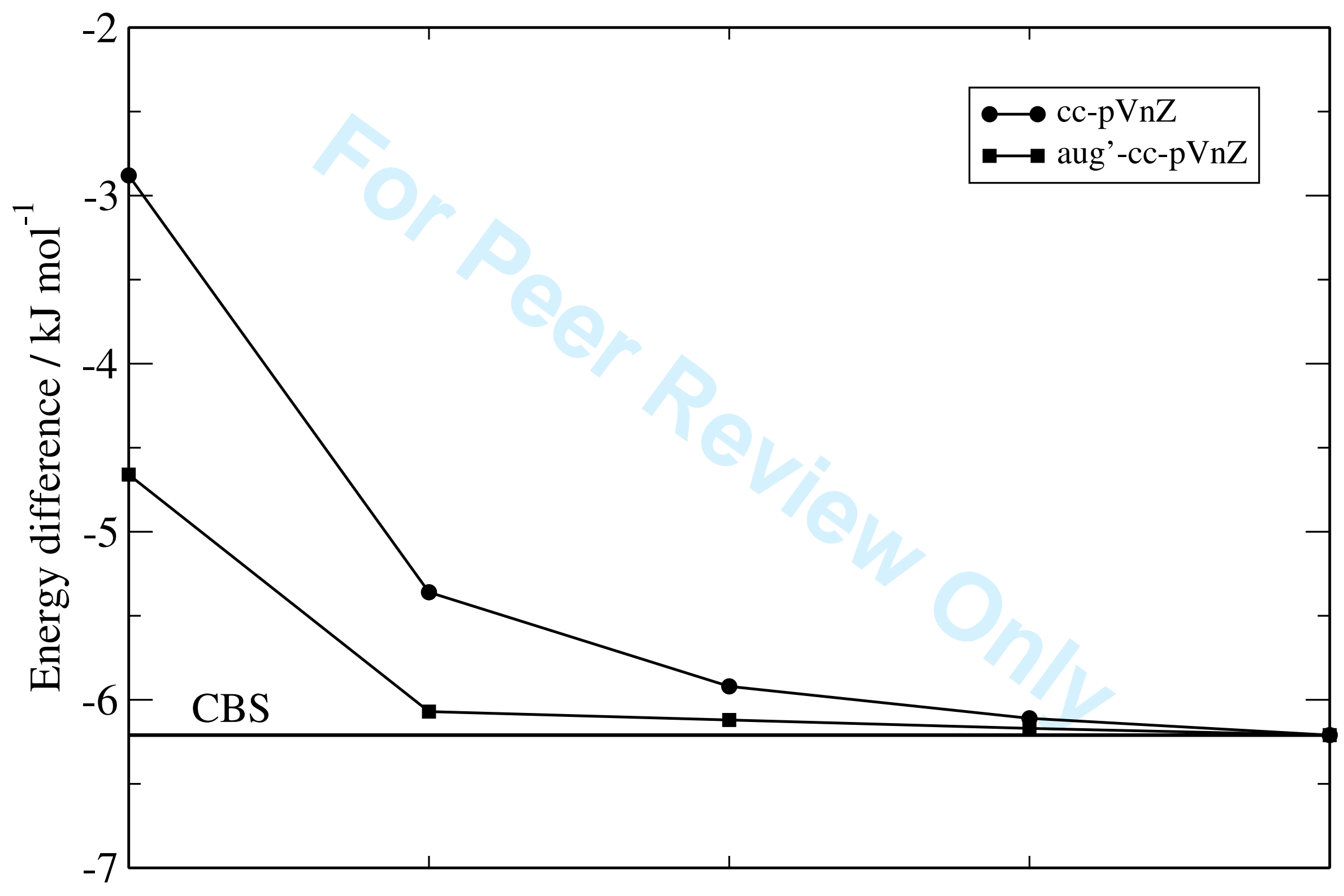

URL: http://mc.manuscriptcentral.com/tandf/tmph 


1
2
3
4
5
6
7
8
9
10
1
1
13
1
15
16
17
18
19
20
2
2
23
2
25
26
2
2
5
5
5
5
5
5
5
5
5
5
5
3
3
3
3
35
3
3
38
39
4

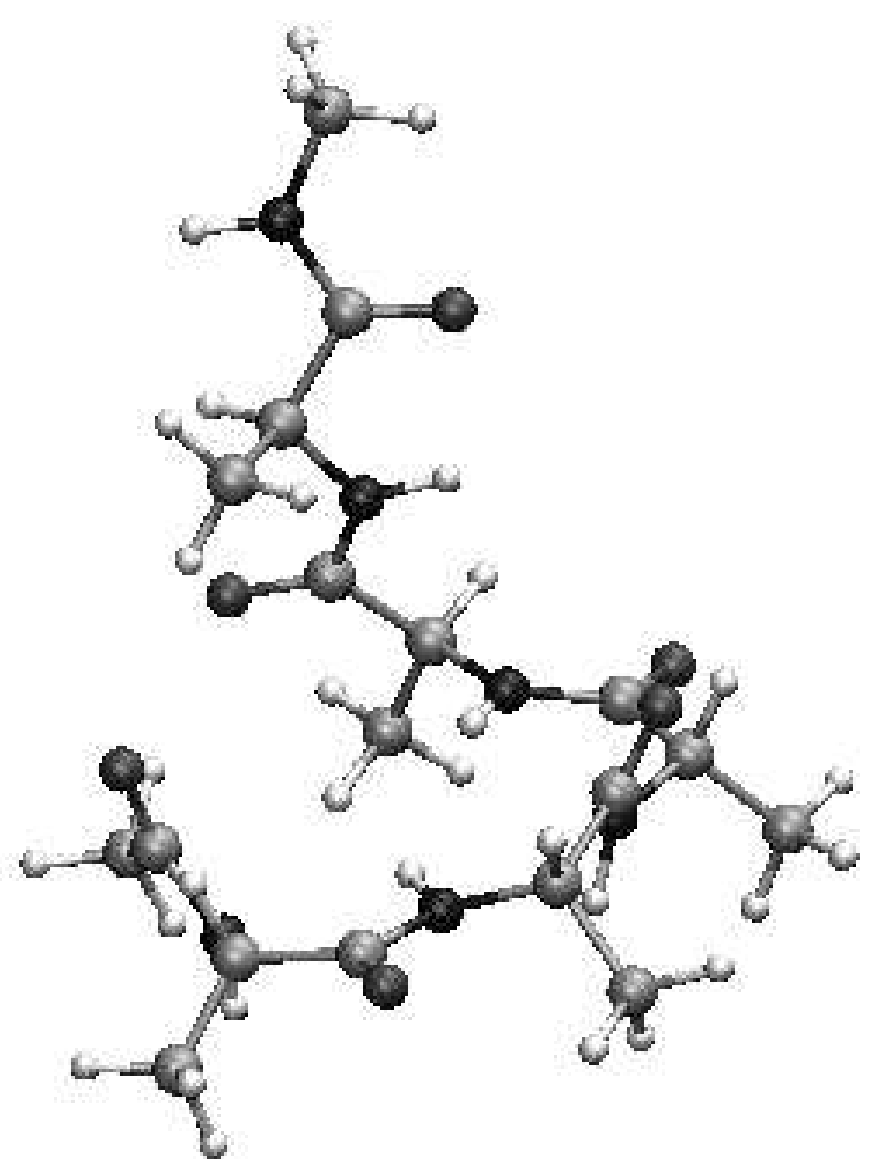

URL: http://mc.manuscriptcentral.com/tandf/tmph 
This file contains:

1.) DF-HF results

2.) DF-MP2 results

3.) DF-LMP2 results

4.) Geometries (xyz format)

Basis sets:

DZ: $\quad c c-p V D Z$

TZ: cc-pVTZ

QZ: $\quad$ cc-pVQZ

5Z: $\quad c c-p V 5 Z$

ADZ: aug-cc-pVDZ

ATZ: aug-cc-pVTZ

(A)DZ: (aug)-cc-pVTZ

(A)TZ: (aug)-cc-pVTZ

(A)QZ: (aug)-cc-pVTZ

(A)5Z: (aug)-cc-pVTZ

The (aug)-cc-pVnZ basis sets do not include diffuse function on $\mathrm{H}$-atoms.

All results obtained with density fitting, using the cc-pVnZ/JKFIT sets for DF-HF

the cc-pVnZ/MP2FIT for MP2/cc-pVnZ, and aug-cc-pVnZ/MP2FIT for both MP2/aug-cc-pVnZ and MP2/(aug)-cc-pVnZ

All relative energies in $\mathrm{kJ} / \mathrm{mol}$

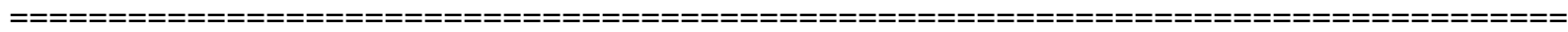

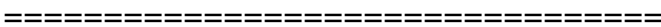

DF-HF results

URL: http://mc.manuscriptcentral.com/tandf/tmph 
$\begin{array}{lllllllllll}D Z & \text { TZ } & \text { QZ } & 5 Z & \text { ADZ } & \text { ATZ } & \text { (A)DZ } & \text { (A)TZ } & \text { (A)QZ } & \text { (A)5Z XPOL } & \text { REF }\end{array}$ Glycine

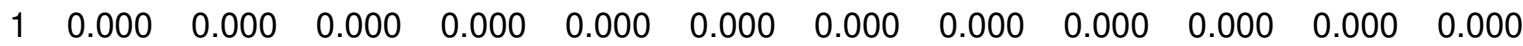

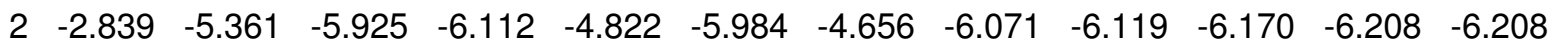

Alanine

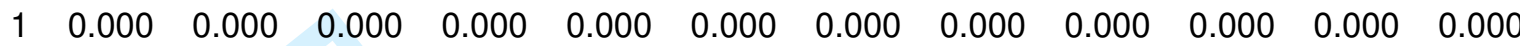

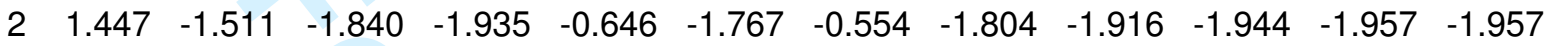

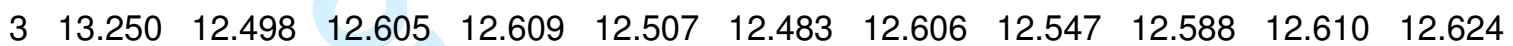
12.624

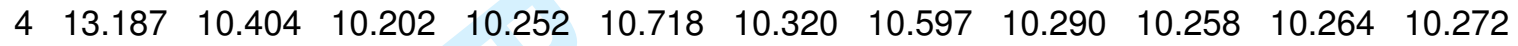
10.272

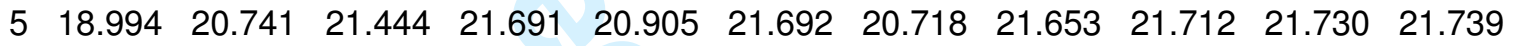
21.739

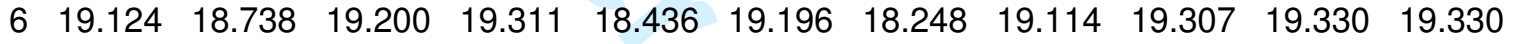
19.330

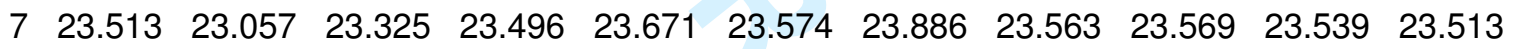
23.513

Serine

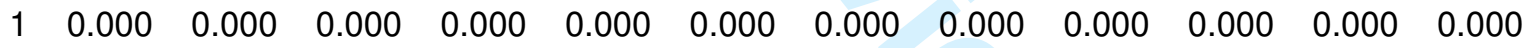

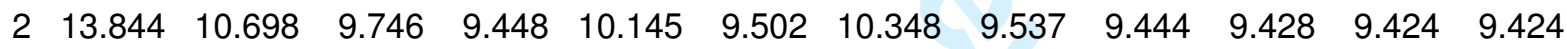

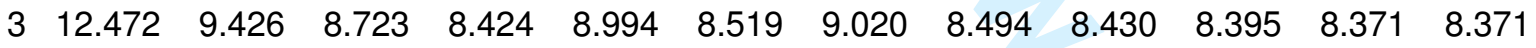

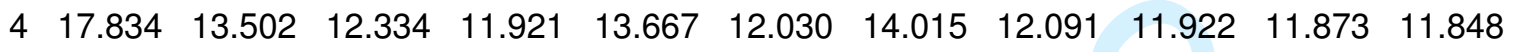
11.848

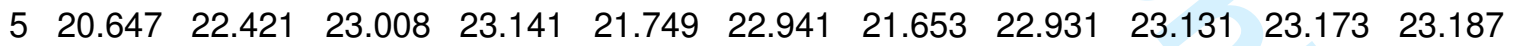
23.187

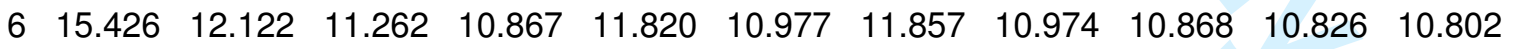
10.802

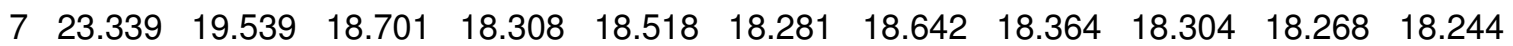
18.244

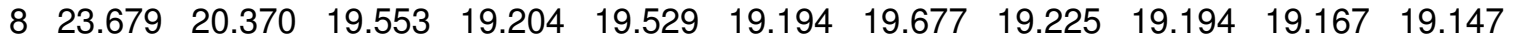
19.147

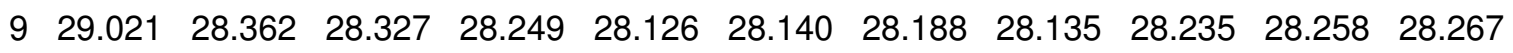
28.267

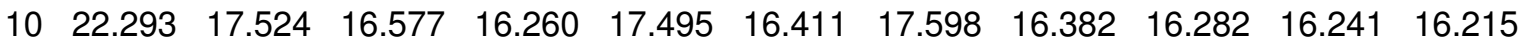


16.215

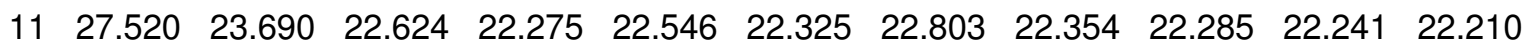
22.210

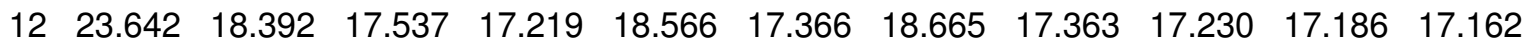
17.162

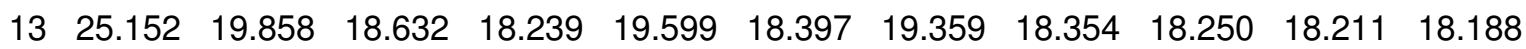
18.188

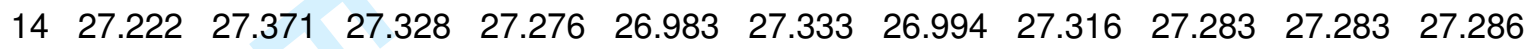
27.286

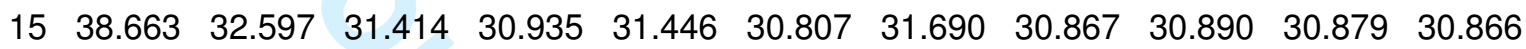
30.866

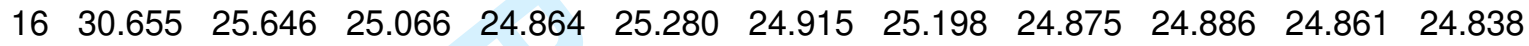
24.838

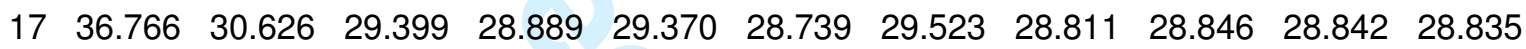
28.835

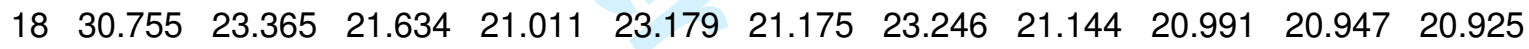
20.925

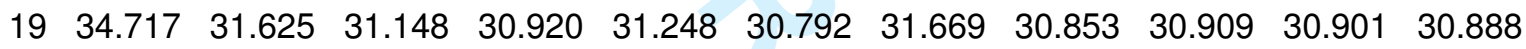
30.888

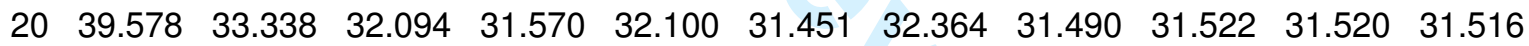
31.516

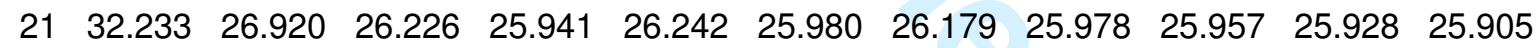
25.905

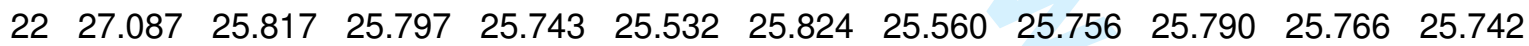
25.742

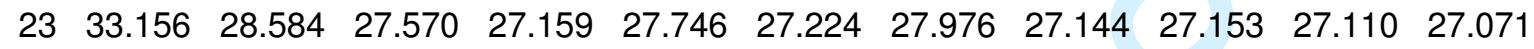
27.071

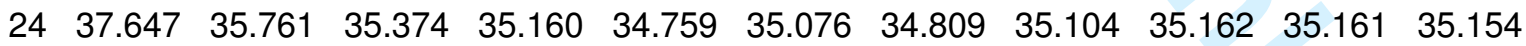
35.154

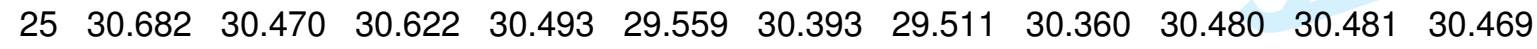
30.469

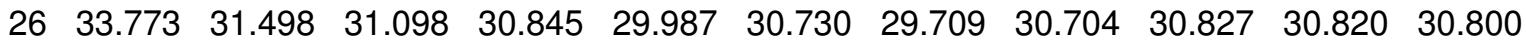
30.800

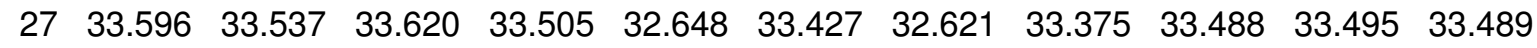
33.489

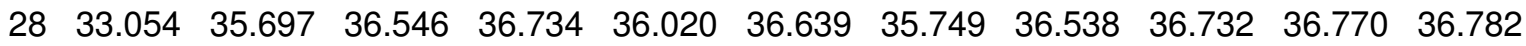
36.782

URL: http://mc.manuscriptcentral.com/tandf/tmph 


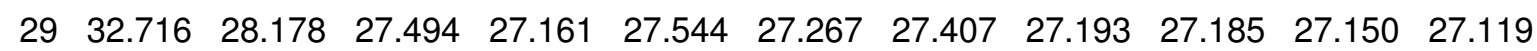
27.119

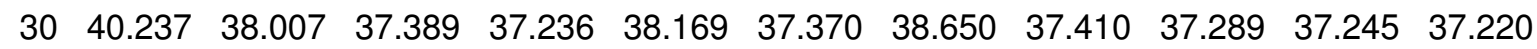
37.220

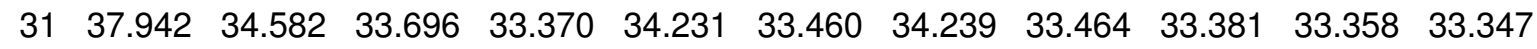
33.347

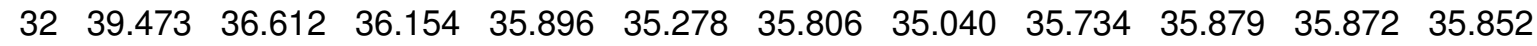
35.852

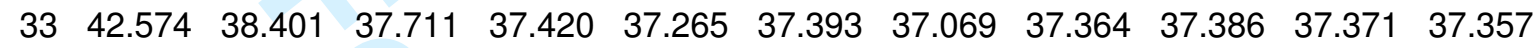
37.357

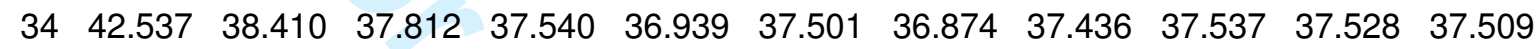
37.509

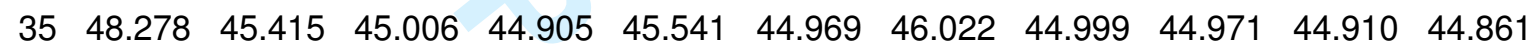
44.861

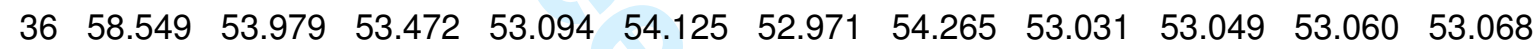
53.068

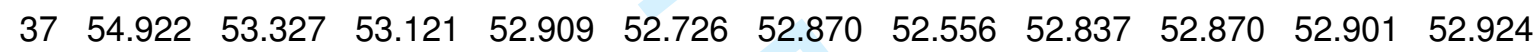
52.924

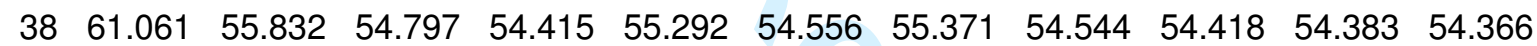
54.366

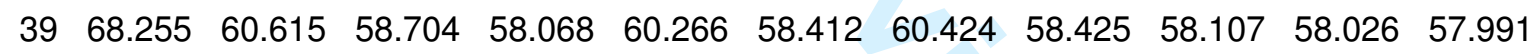
57.991

Cysteine

$\begin{array}{lllllllllllll}1 & 0.000 & 0.000 & 0.000 & 0.000 & 0.000 & 0.000 & 0.000 & 0.000 & 0.000 & 0.000 & 0.000 & 0.000\end{array}$

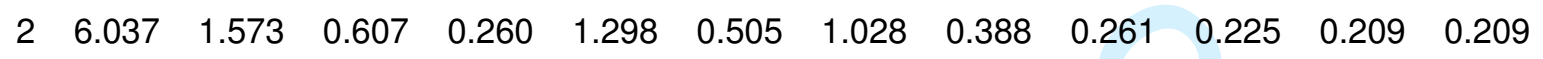

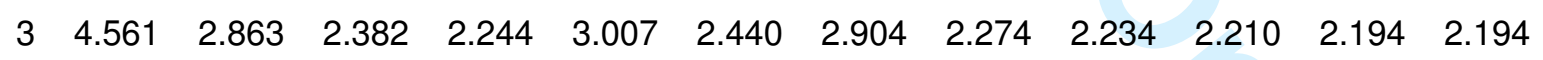

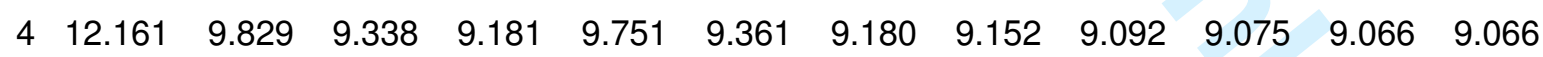

$\begin{array}{lllllllllllll}5 & 6.315 & 4.609 & 4.200 & 4.134 & 4.500 & 4.307 & 4.387 & 4.217 & 4.144 & 4.124 & 4.114 & 4.114\end{array}$

$\begin{array}{lllllllllllll}6 & 7.893 & 6.097 & 5.630 & 5.466 & 6.107 & 5.687 & 5.750 & 5.570 & 5.443 & 5.426 & 5.424 & 5.424\end{array}$

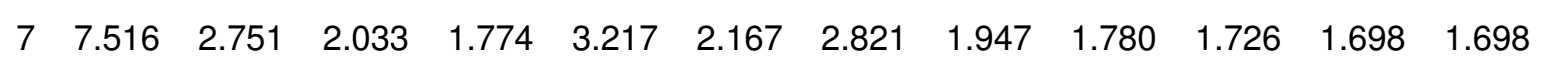

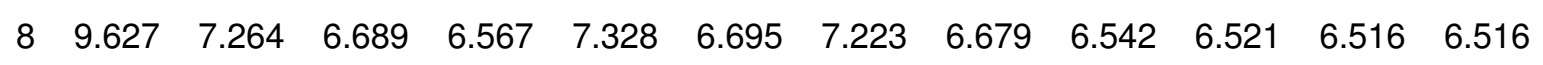

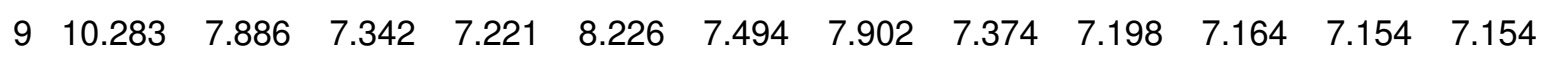

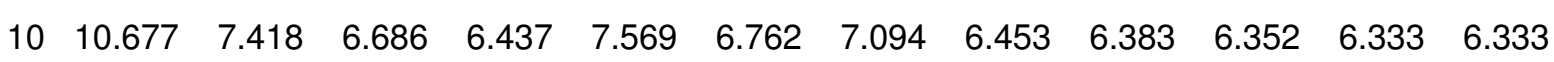

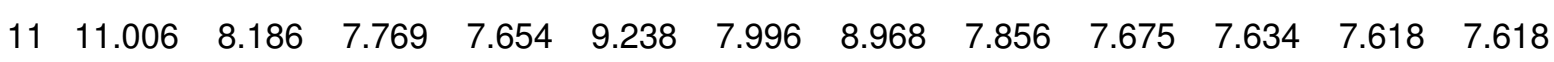




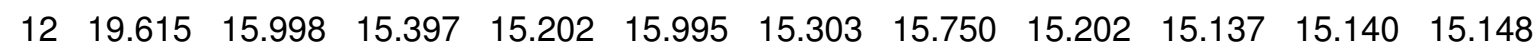
15.148

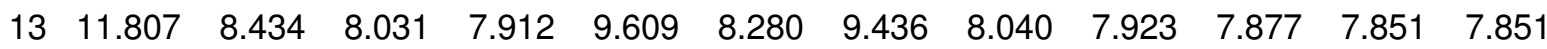

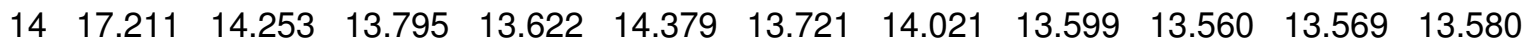
13.580

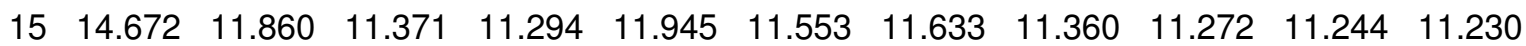
11.230

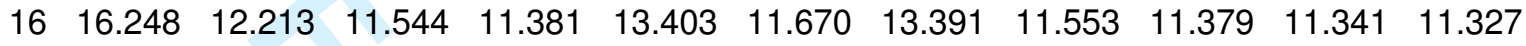
11.327

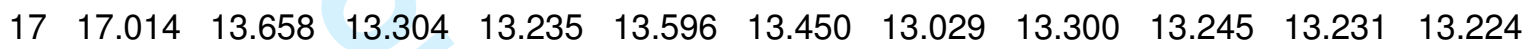
13.224

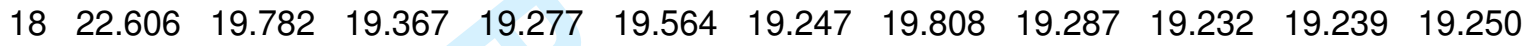
19.250

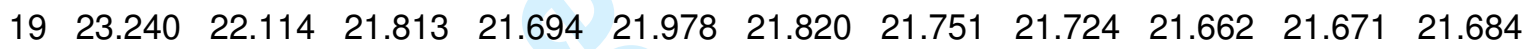
21.684

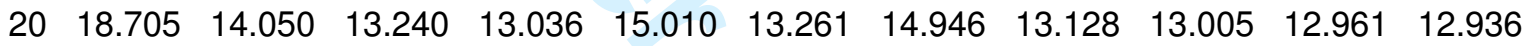
12.936

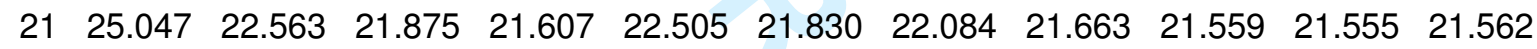
21.562

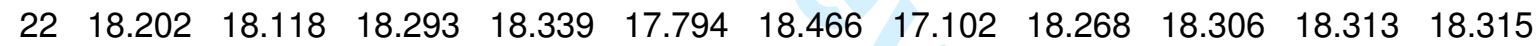
18.315

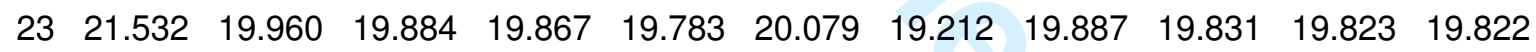
19.822

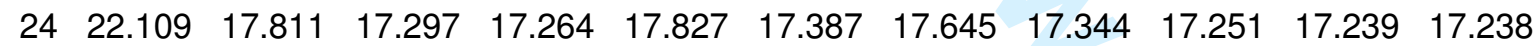
17.238

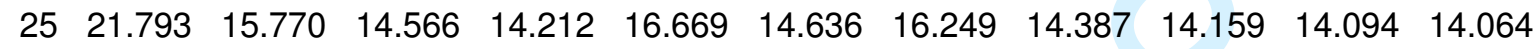
14.064

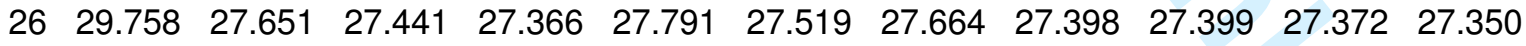
27.350

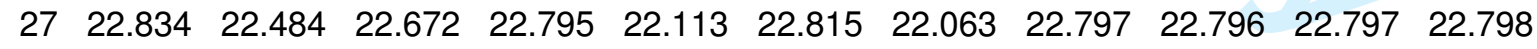
22.798

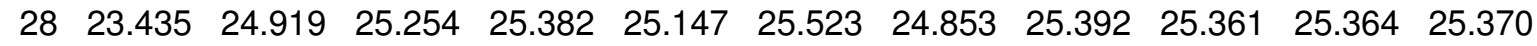
25.370

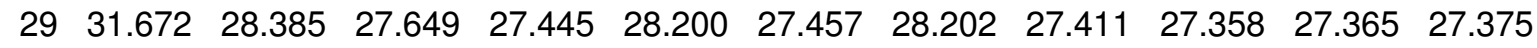
27.375

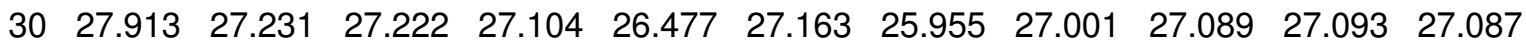
27.087

URL: http://mc.manuscriptcentral.com/tandf/tmph 


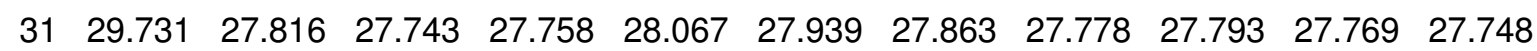
27.748

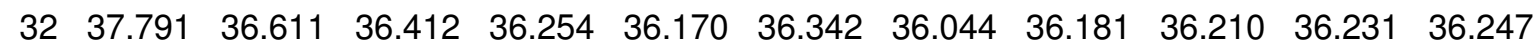
36.247

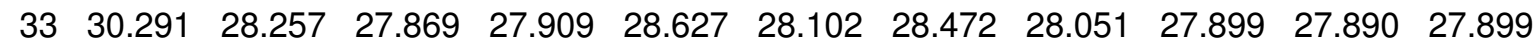
27.899

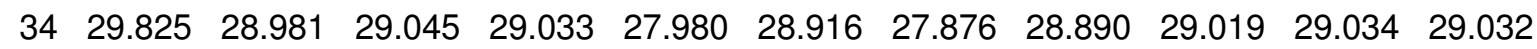
29.032

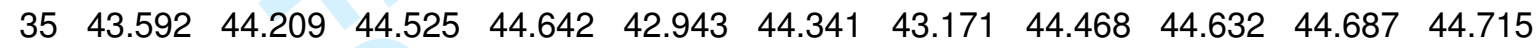
44.715

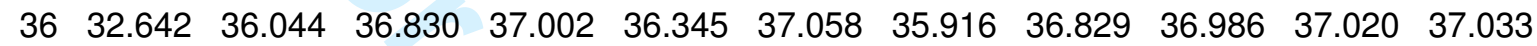
37.033

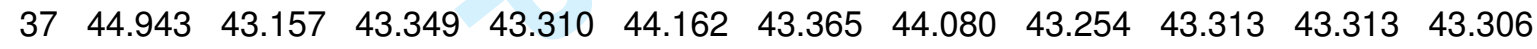
43.306

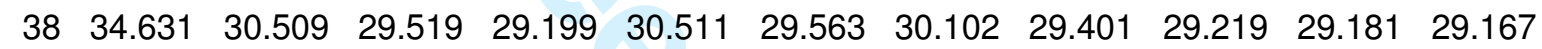
29.167

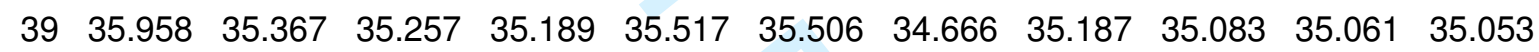
35.053

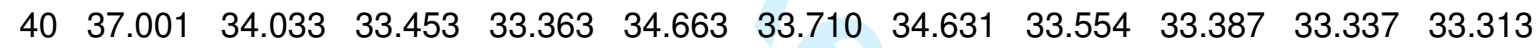
33.313

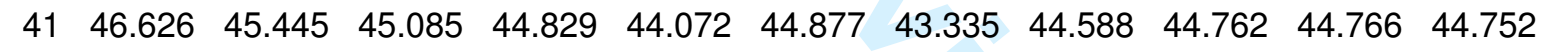
44.752

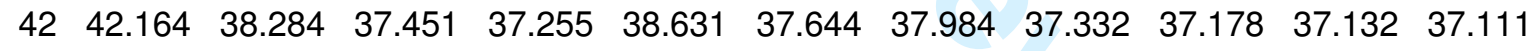
37.111

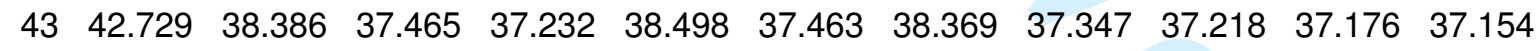
37.154

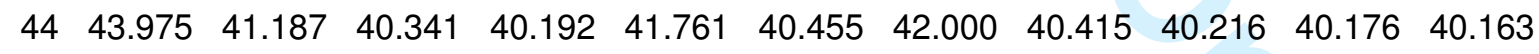
40.163

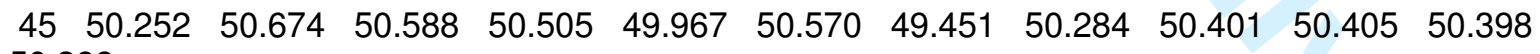
50.398

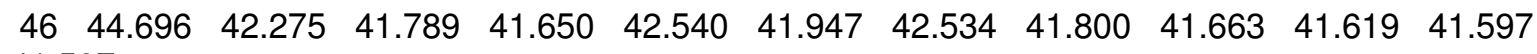
41.597

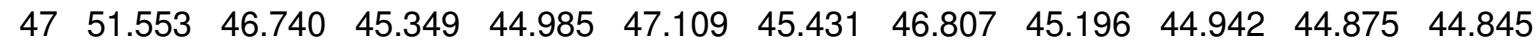
44.845

MAD

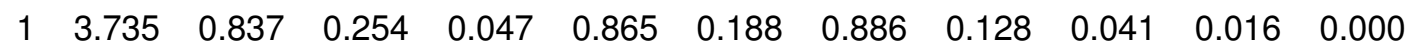
$\operatorname{Max} A D$

URL: http://mc.manuscriptcentral.com/tandf/tmph 


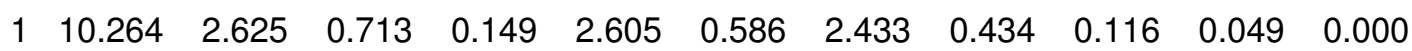

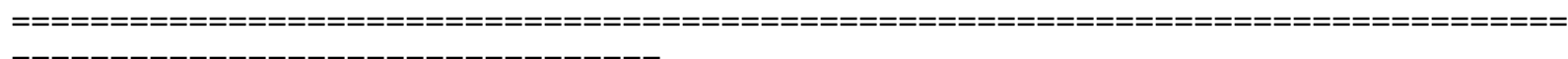

DF-MP2 results (using DF-HF reference functions)

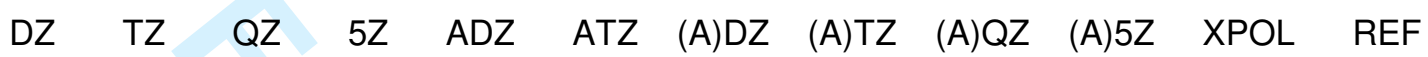
Glycine

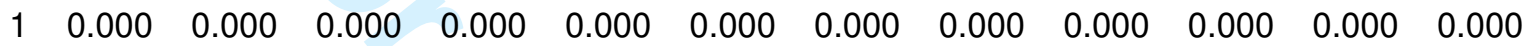

$\begin{array}{lllllllllllll}2 & 7.957 & 6.321 & 5.194 & 4.900 & 7.348 & 5.729 & 7.155 & 5.418 & 4.895 & 4.783 & 4.680 & 4.680\end{array}$

Alanine

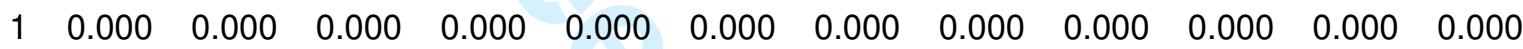

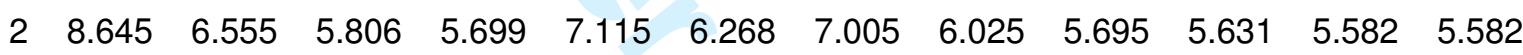

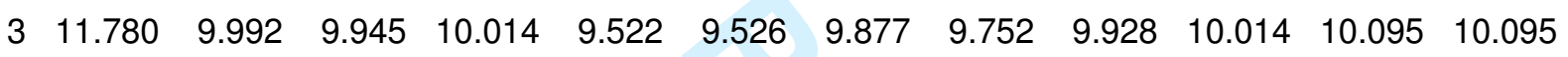

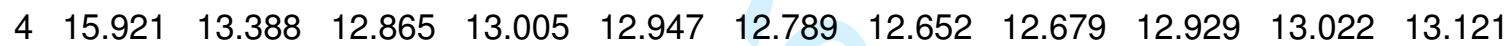
13.121

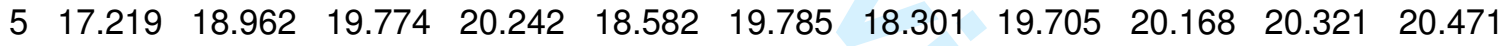
20.471

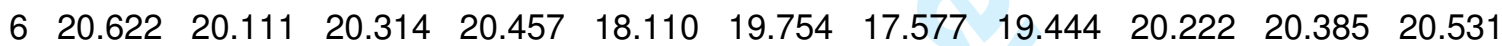
20.531

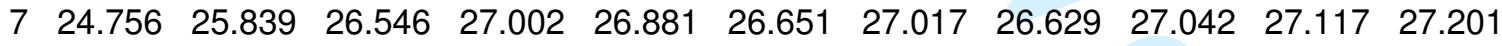
27.201

Serine

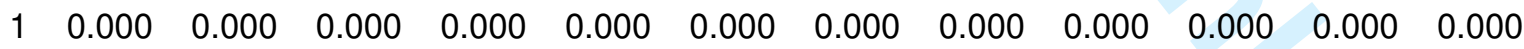

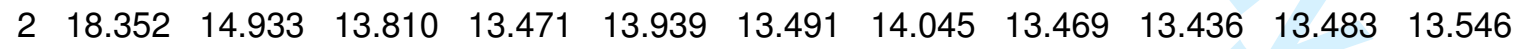
13.546

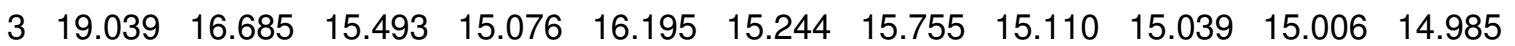
14.985

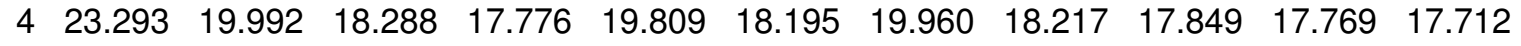
17.712

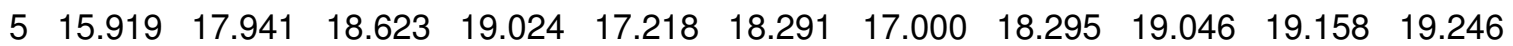
19.246

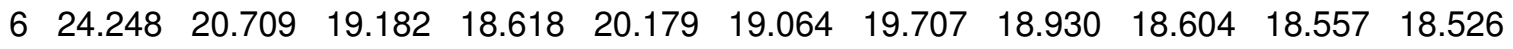


18.526

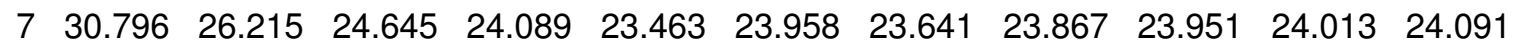
24.091

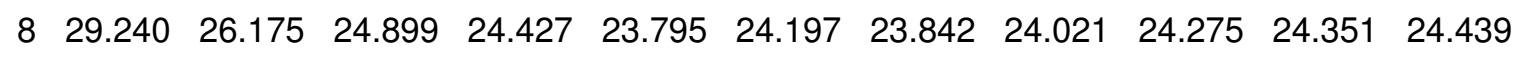
24.439

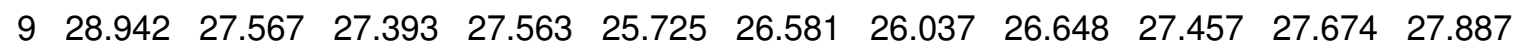
27.887

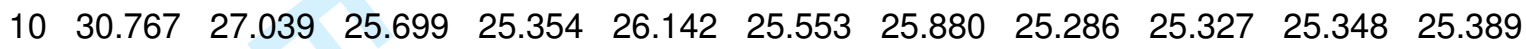
25.389

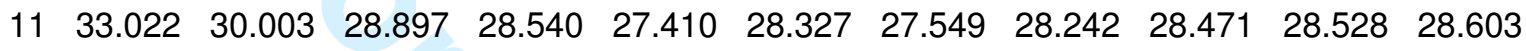
28.603

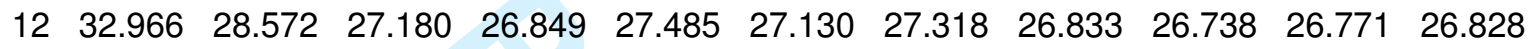
26.828

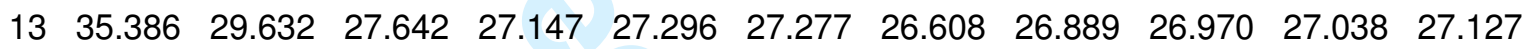
27.127

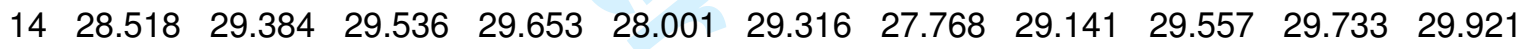
29.921

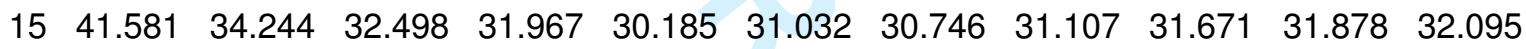
32.095

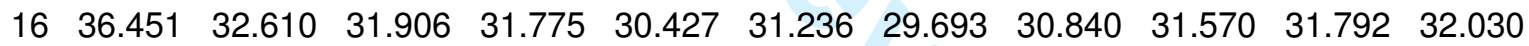
32.030

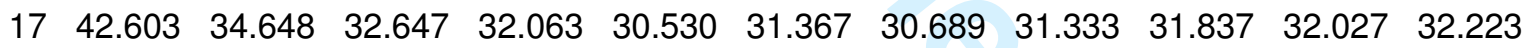
32.223

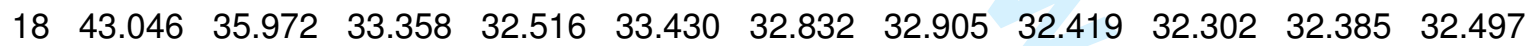
32.497

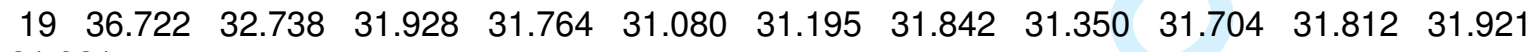
31.921

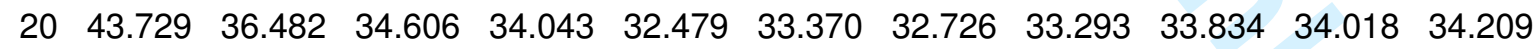
34.209

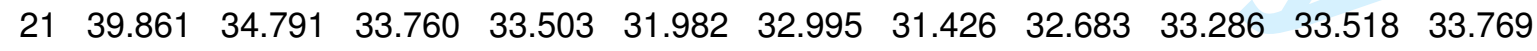
33.769

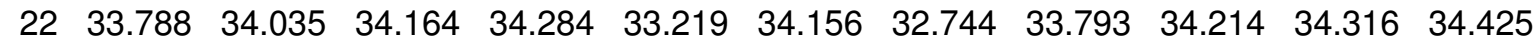
34.425

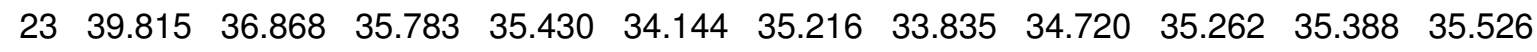
35.526

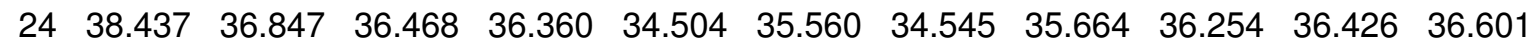
36.601

URL: http://mc.manuscriptcentral.com/tandf/tmph 


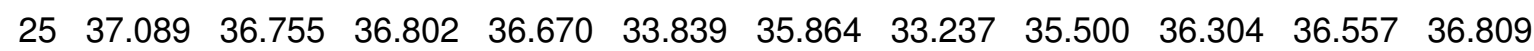
36.809

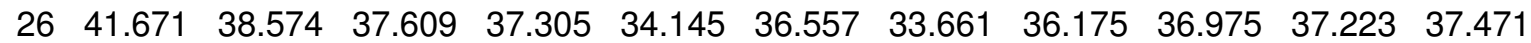
37.471

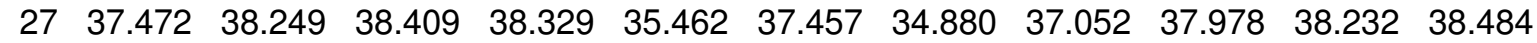
38.484

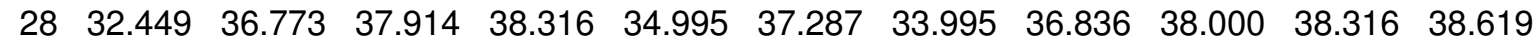
38.619

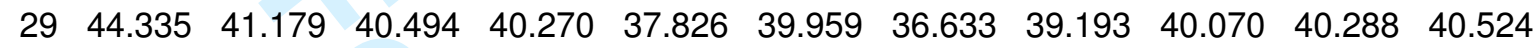
40.524

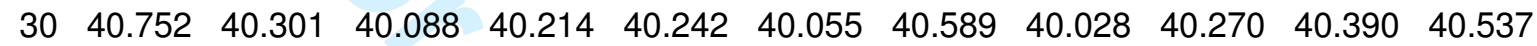
40.537

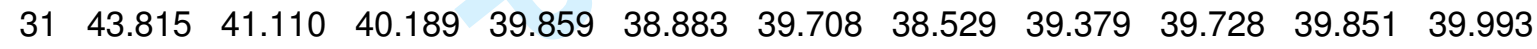
39.993

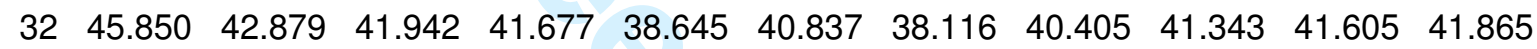
41.865

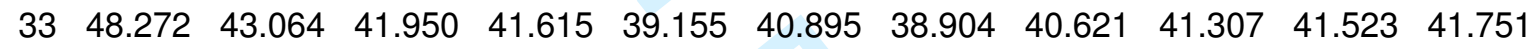
41.751

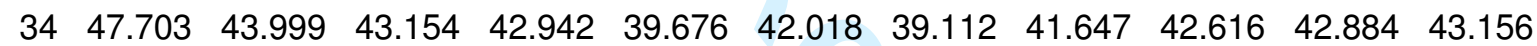
43.156

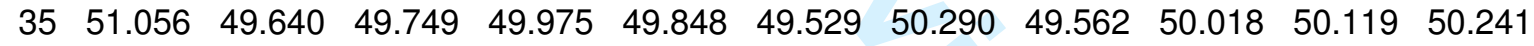
50.241

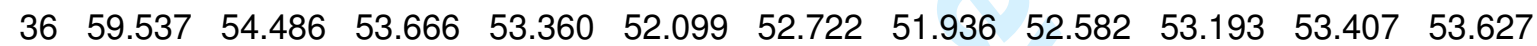
53.627

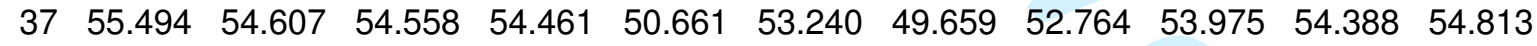
54.813

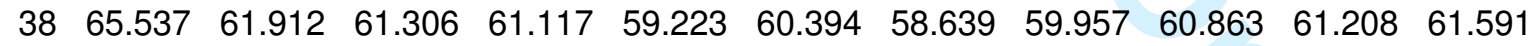
61.591

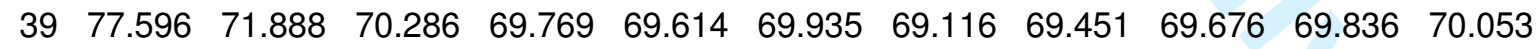
70.053

\section{Cysteine}

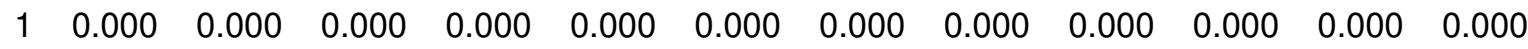

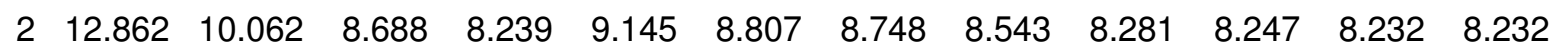

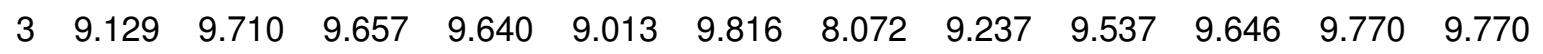

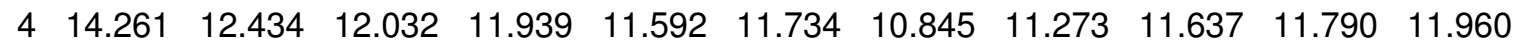
11.960 


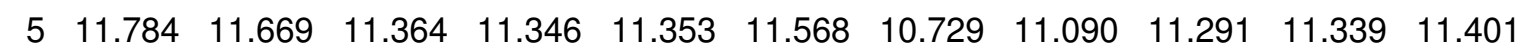
11.401

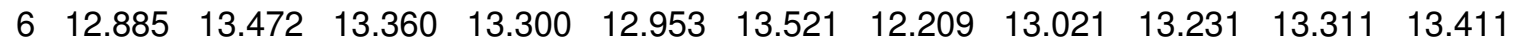
13.411

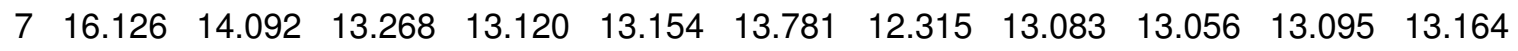
13.164

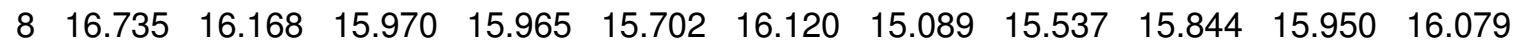
16.079

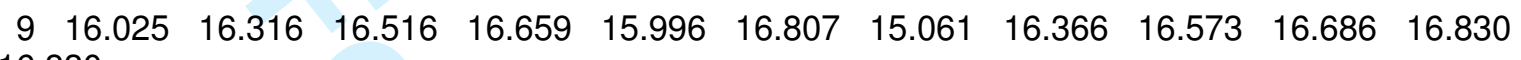
16.830

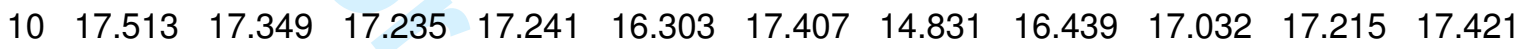
17.421

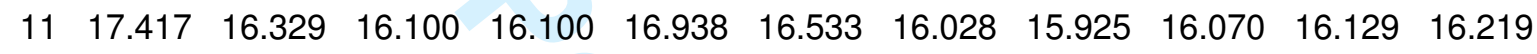
16.219

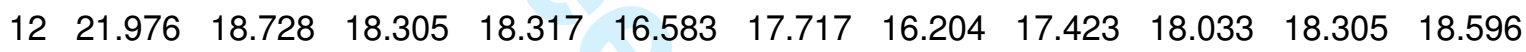
18.596

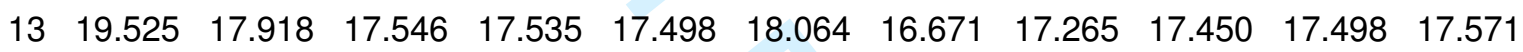
17.571

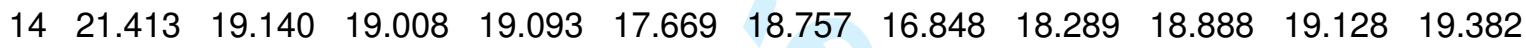
19.382

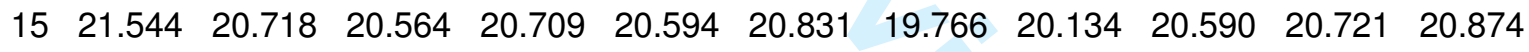
20.874

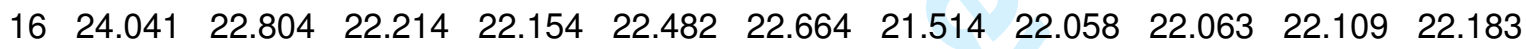
22.183

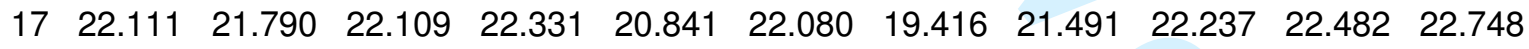
22.748

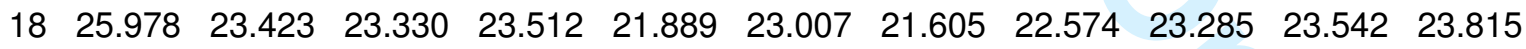
23.815

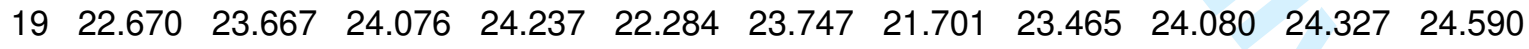
24.590

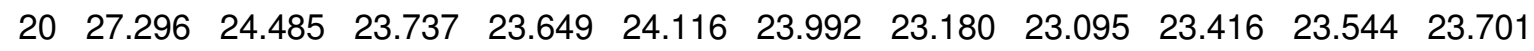
23.701

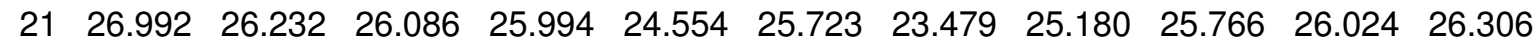
26.306

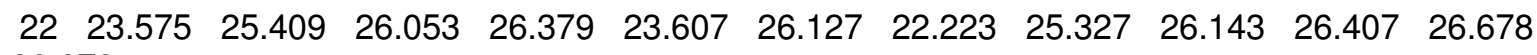
26.678

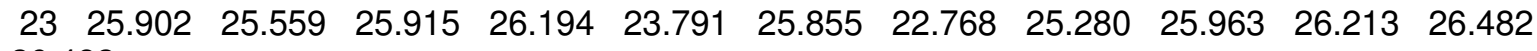
26.482

URL: http://mc.manuscriptcentral.com/tandf/tmph 


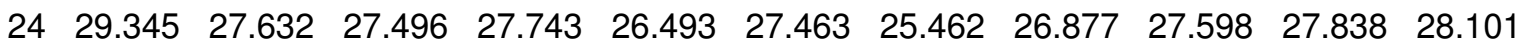
28.101

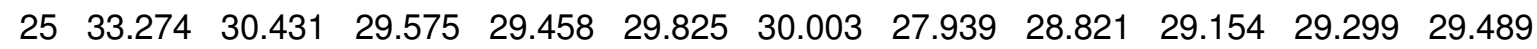
29.489

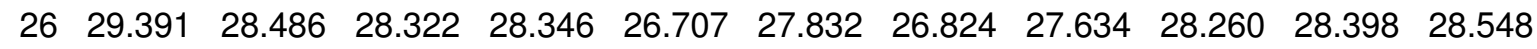
28.548

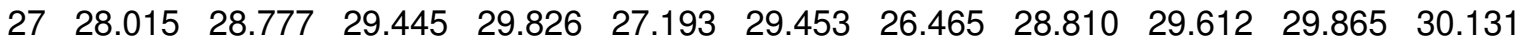
30.131

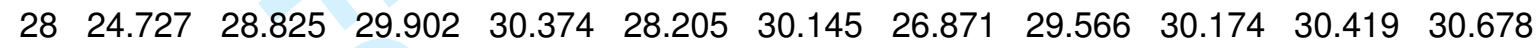
30.678

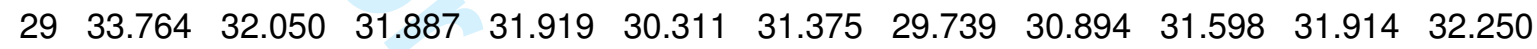
32.250

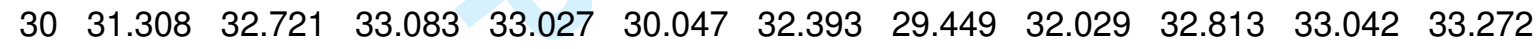
33.272

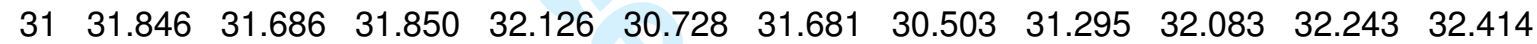
32.414

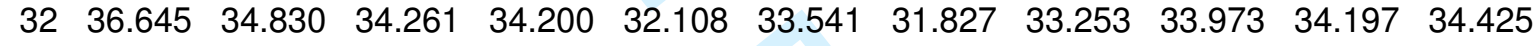
34.425

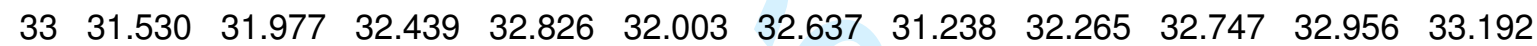
33.192

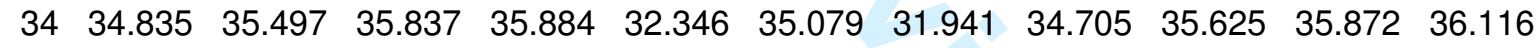
36.116

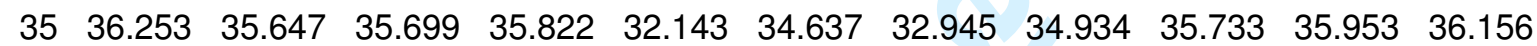
36.156

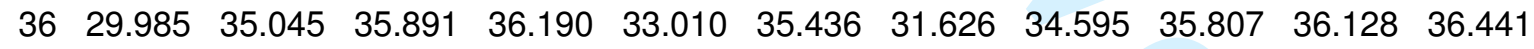
36.441

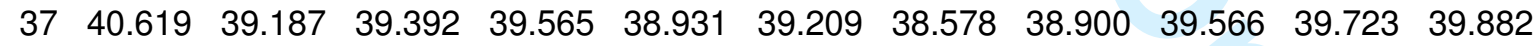
39.882

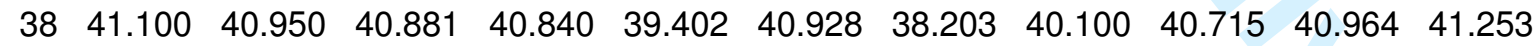
41.253

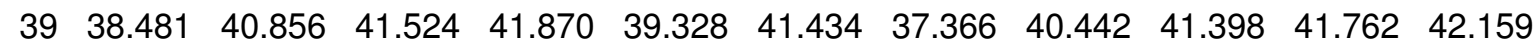
42.159

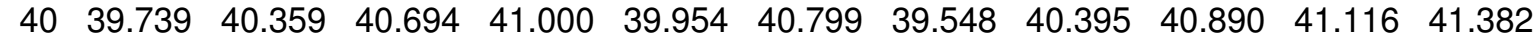
41.382

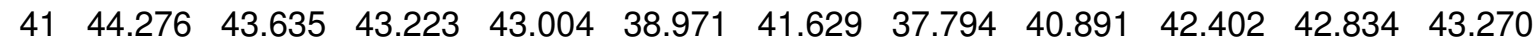
43.270

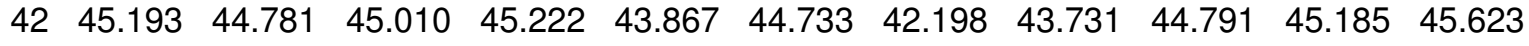
45.623

URL: http://mc.manuscriptcentral.com/tandf/tmph 


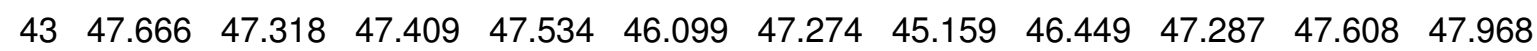
47.968

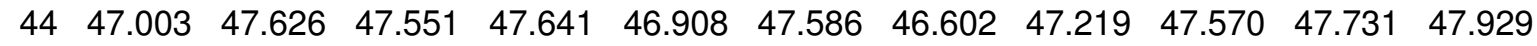
47.929

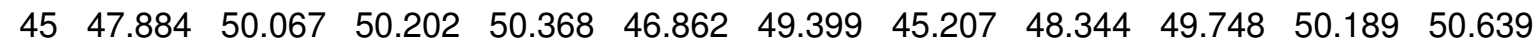
50.639

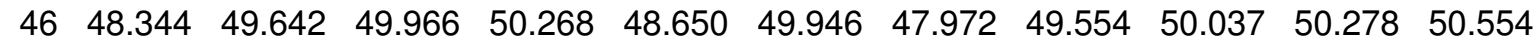
50.554

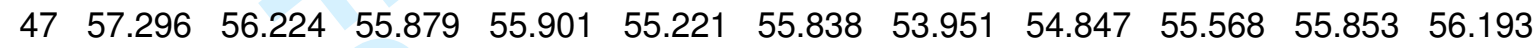
56.193

MAD

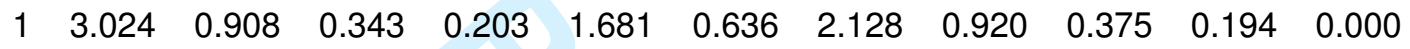

$\operatorname{Max} A D$

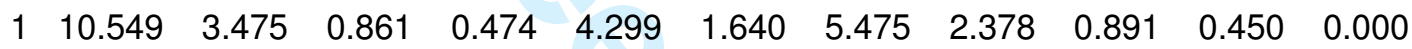

DF-LMP2 results, standard NLMP/NPA domains, npasel=0.03, DF-HF reference functions

Last column: LMP2(ext)/(A)TZ

LMP2(ext)

Glycine

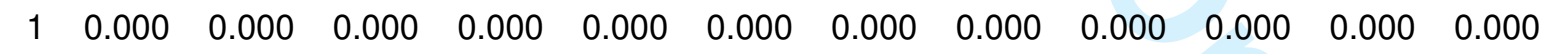
0.000

$\begin{array}{lllllllllllll}2 & 5.041 & 3.953 & 4.048 & 4.277 & 4.455 & 4.286 & 4.616 & 4.155 & 4.379 & 4.464 & 4.568 & 4.680\end{array}$ 4.595

Alanine

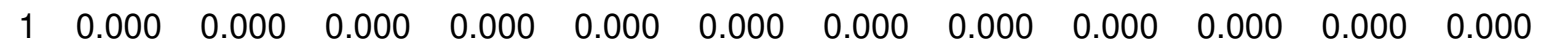
0.000

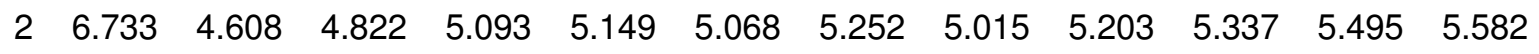
5.345

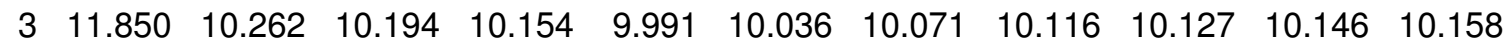
10.09510 .071 


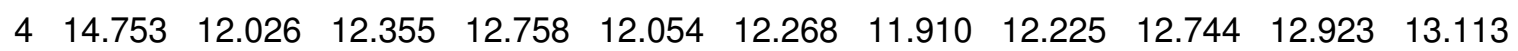
13.12112 .338

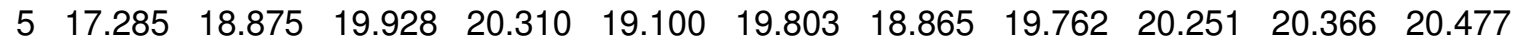
20.47119 .669

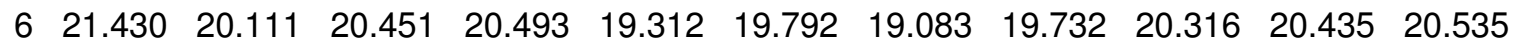
20.53119 .675

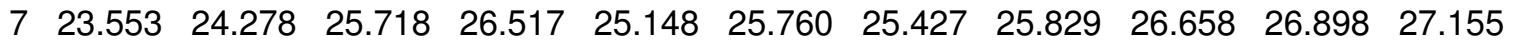
27.20126 .020

Serine

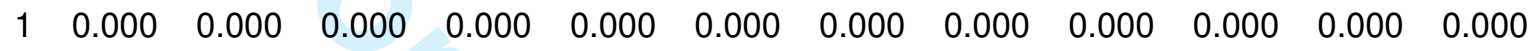
0.000

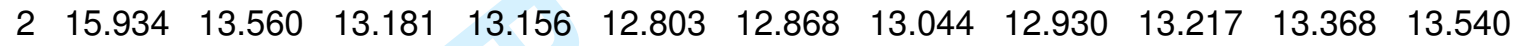
13.54613 .116

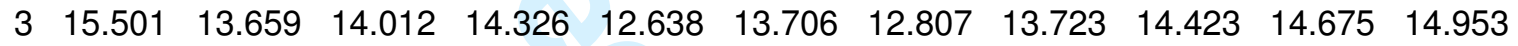
14.98514 .257

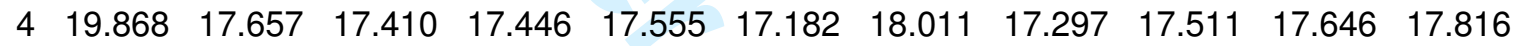
17.71217 .608

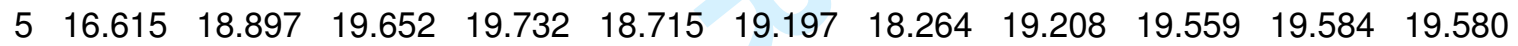
$19.246 \quad 18.943$

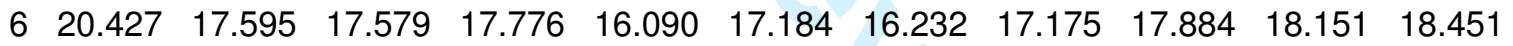
18.52617 .925

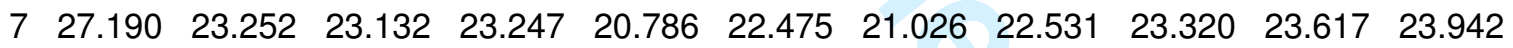
24.09123 .283

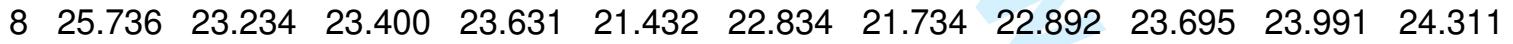
24.43923 .463

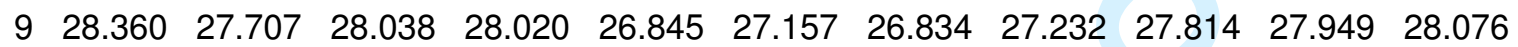
27.88727 .130

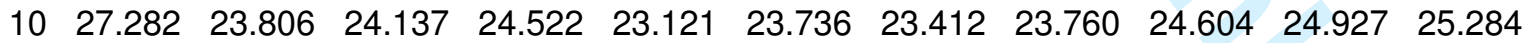
25.38924 .247

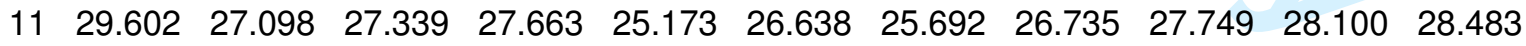
28.60327 .331

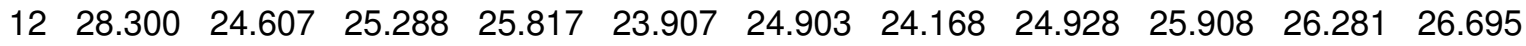
26.82825 .600

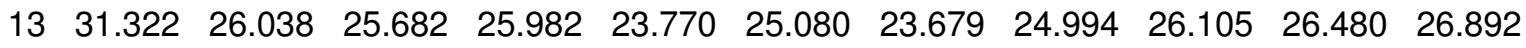
$27.127 \quad 25.812$

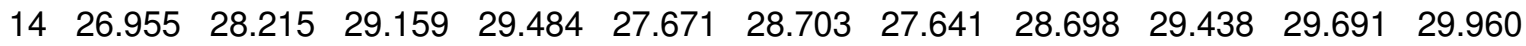
29.92128 .774 


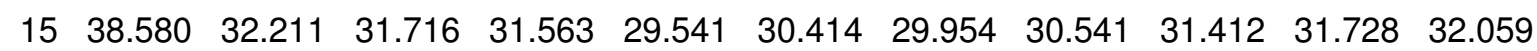
$32.095 \quad 30.895$

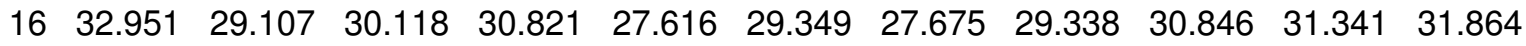
32.03029 .876

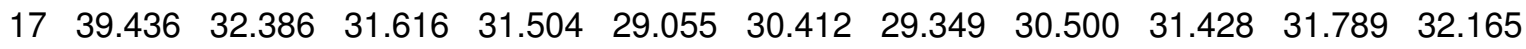
$32.223 \quad 31.050$

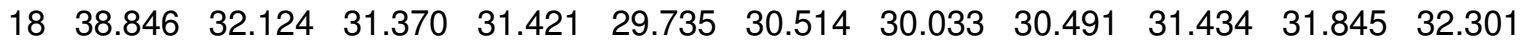
$32.497 \quad 31.289$

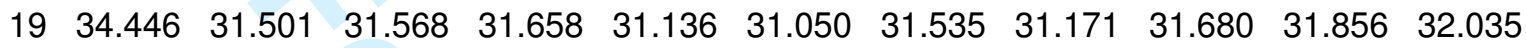
31.92131 .296

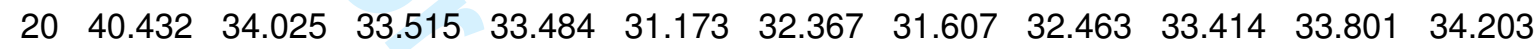
34.20932 .911

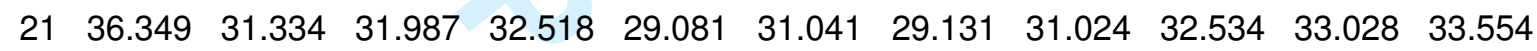
33.76931 .736

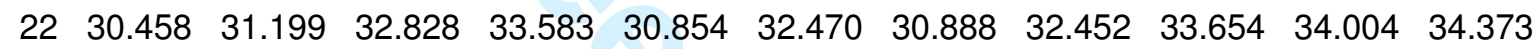
34.42532 .819

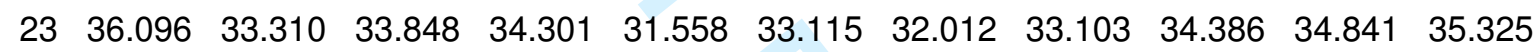
35.52633 .786

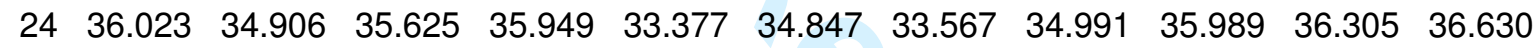
36.60135 .294

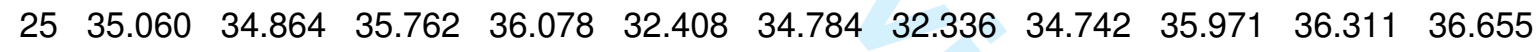
36.80935 .302

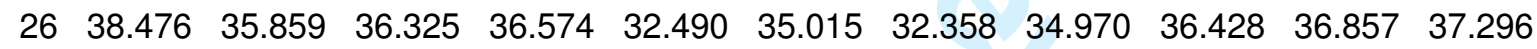
37.47135 .549

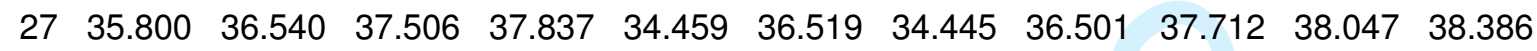
38.48436 .898

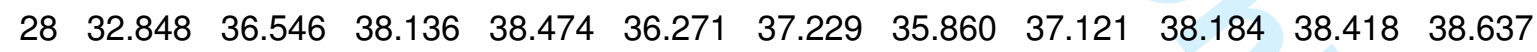
38.61937 .046

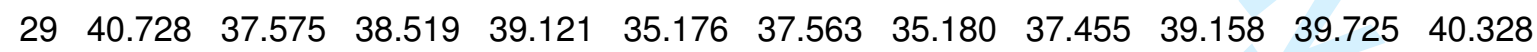
40.52438 .243

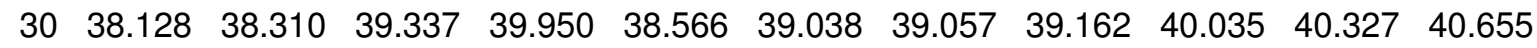
$40.537 \quad 39.345$

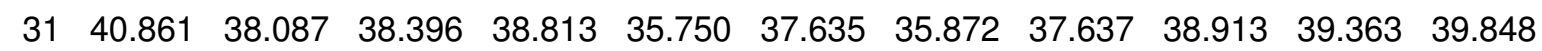
$39.993 \quad 38.494$

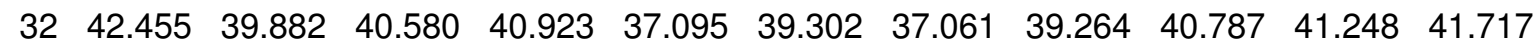
41.86539 .747

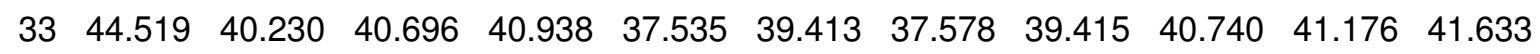


41.75139 .918

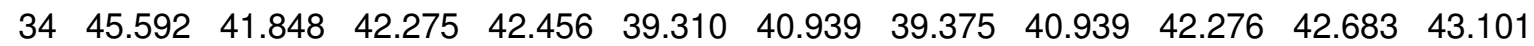
$43.156 \quad 41.209$

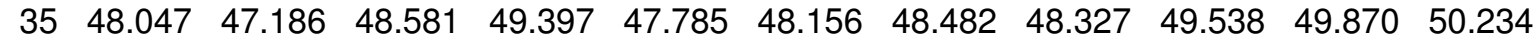
50.24148 .492

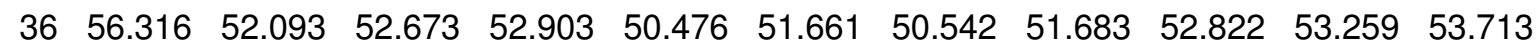
$53.627 \quad 52.334$

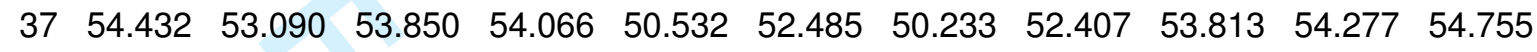
54.81352 .881

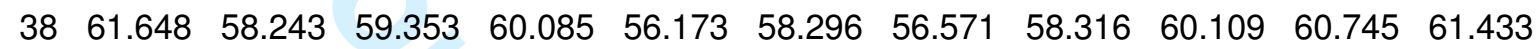
61.59159 .008

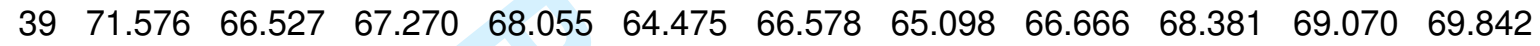
70.05367 .740

Cysteine

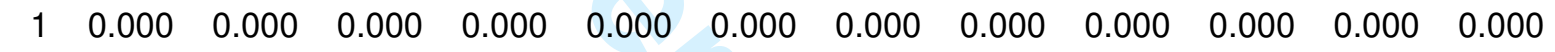
0.000

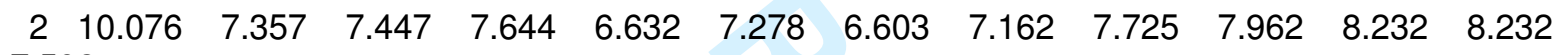
7.592

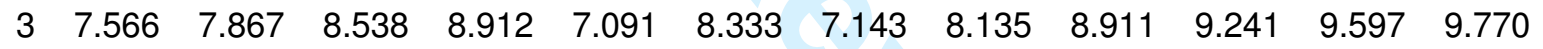
8.657

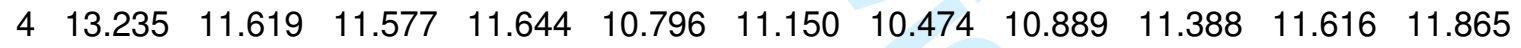
11.96011 .136

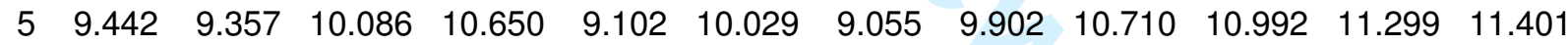
10.185

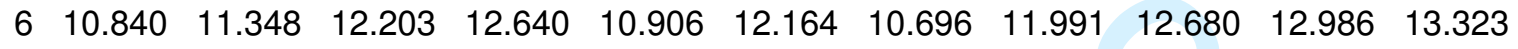
13.41112 .497

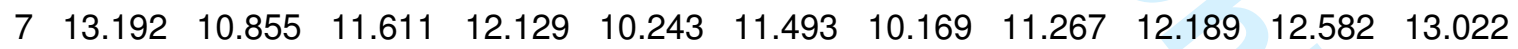
13.16411 .981

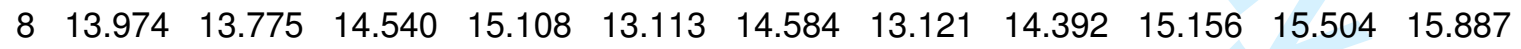
16.07915 .033

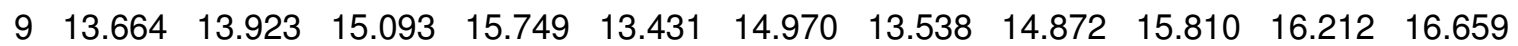
16.83015 .562

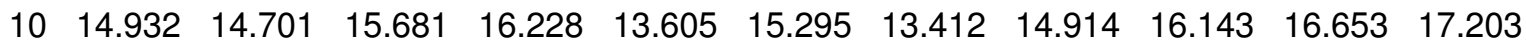
17.42115 .645

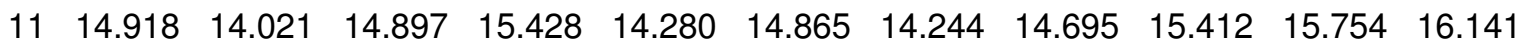
16.21915 .151

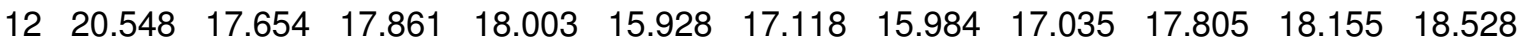


$18.596 \quad 17.344$

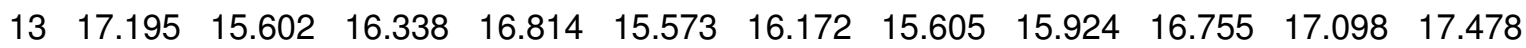
17.57116 .426

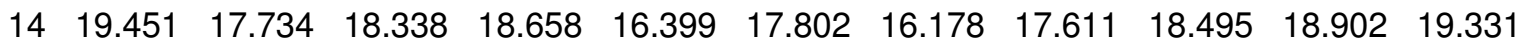
19.38218 .056

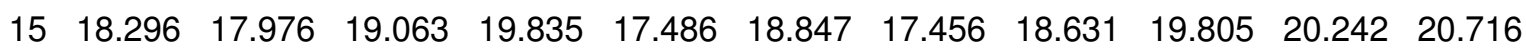
20.87419 .091

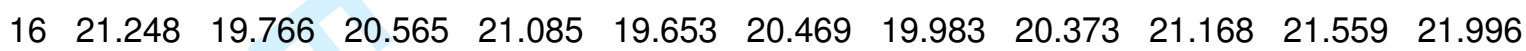
22.18321 .090

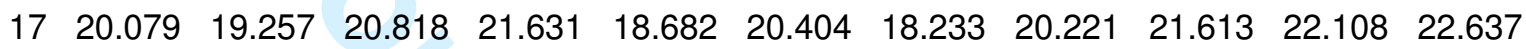
$22.748 \quad 20.597$

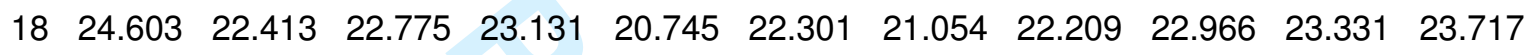
23.81522 .582

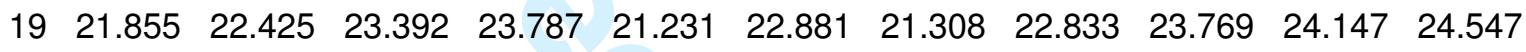
$24.590 \quad 23.219$

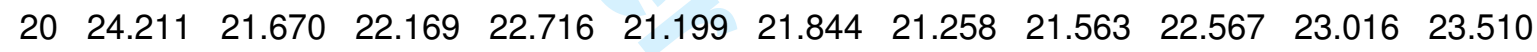
23.70122 .257

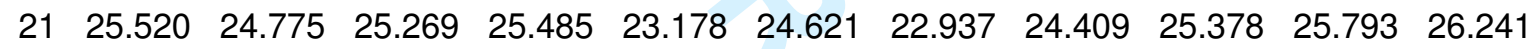
$26.306 \quad 24.919$

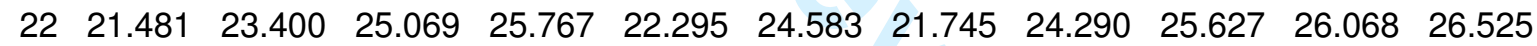
26.67824 .753

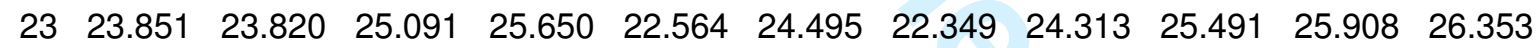
26.48224 .726

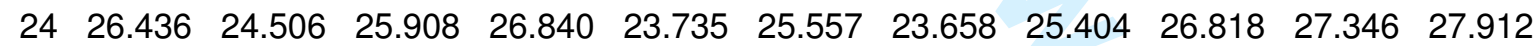
28.10125 .944

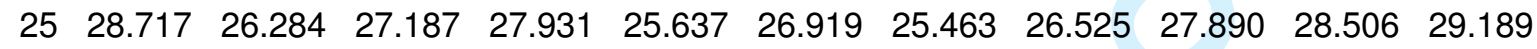
29.48927 .570

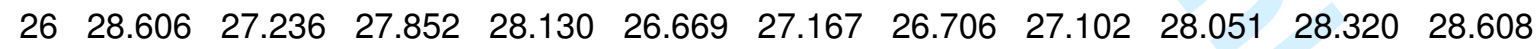
28.54827 .191

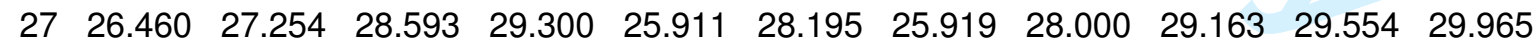
30.13128 .397

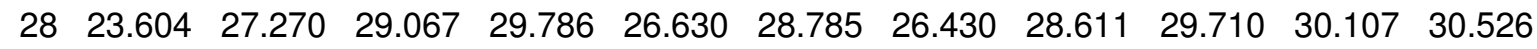
30.67829 .035

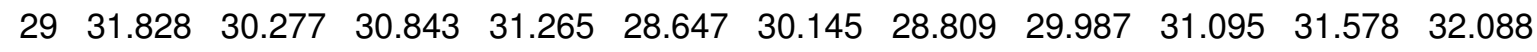
$32.250 \quad 30.591$

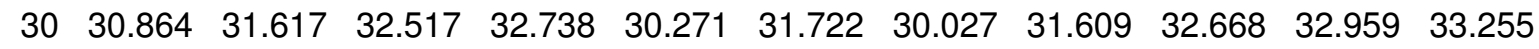
33.27231 .852 


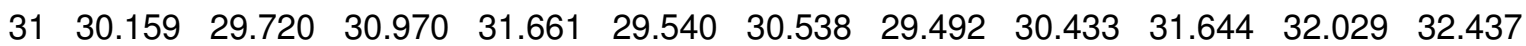
32.41430 .702

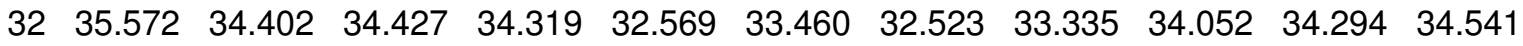
34.42533 .640

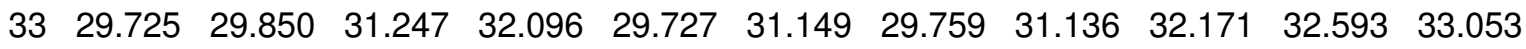
33.19231 .531

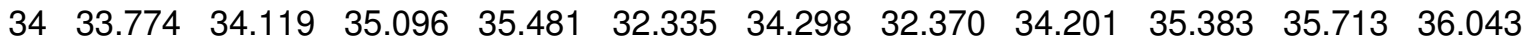
$36.116 \quad 34.510$

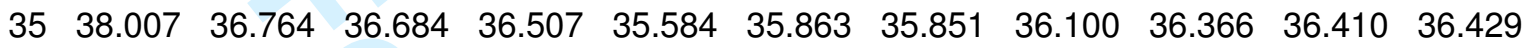
36.15635 .658

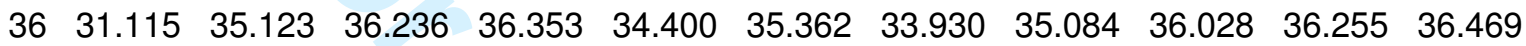
36.44135 .026

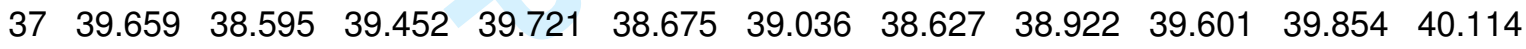
39.88239 .203

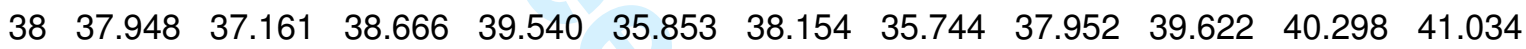
$41.253 \quad 38.844$

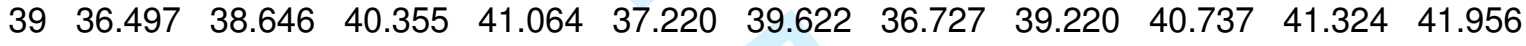
42.15939 .823

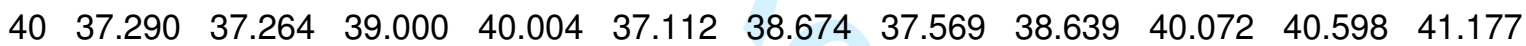
41.38239 .212

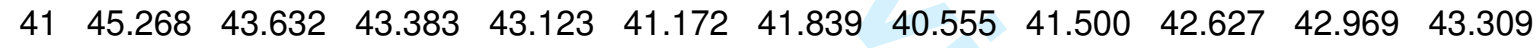
43.27041 .456

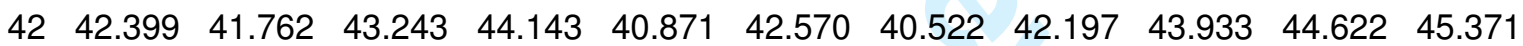
$45.623 \quad 42.850$

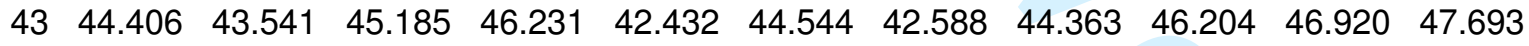
$47.968 \quad 45.271$

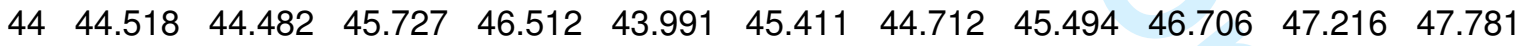
$47.929 \quad 46.218$

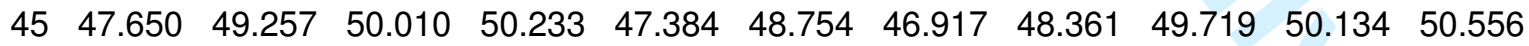
50.63948 .491

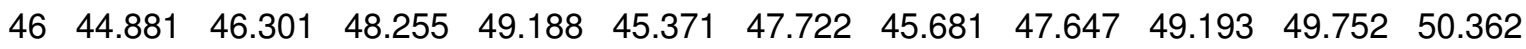
$50.554 \quad 48.440$

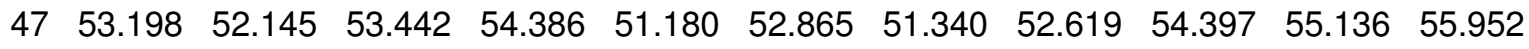
56.19353 .622

MAD

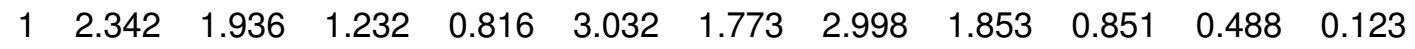
1.410 


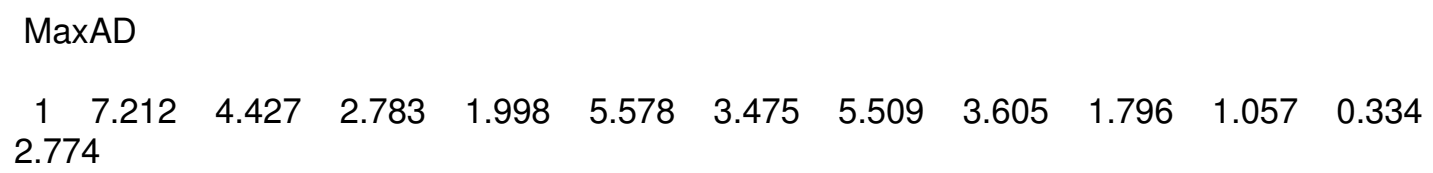

URL: http://mc.manuscriptcentral.com/tandf/tmph 


\title{
$\begin{array}{llll}H & -2.335331 & 2.564465 & 0.029241\end{array}$
}

glycine_2 -455.33656092531 a.u.

$\begin{array}{llll}\text { C } & 3.408842 & 0.915890 & 0.000064\end{array}$

$\begin{array}{llll}\text { C } & 2.340217 & -0.161251 & 0.000005\end{array}$

$\begin{array}{llll}\mathrm{O} & 2.612265 & -1.371075 & 0.000060\end{array}$

$\begin{array}{llll}H & 4.041608 & 0.780273 & 0.889226\end{array}$

$\begin{array}{llll}H & 4.041946 & 0.780036 & -0.888820\end{array}$

H $\quad 2.991492 \quad 1.933220 \quad-0.000146$

$\begin{array}{llll}N & 1.048372 & 0.279676 & -0.000106\end{array}$

$\begin{array}{llll}\text { C } & -0.049249 & -0.659777 & -0.000081\end{array}$

$\begin{array}{llll}\text { C } & -1.356984 & 0.129111 & -0.000043\end{array}$

$\begin{array}{lllll}O & -1.382383 & 1.368707 & -0.000024\end{array}$

$\begin{array}{llll}H & 0.800691 & 1.264838 & -0.000066\end{array}$

$\begin{array}{llll}H & -0.001905 & -1.315939 & -0.886760\end{array}$

$\begin{array}{llll}\mathrm{H} & -0.001854 & -1.315948 & 0.886590\end{array}$

N $\quad-2.490703 \quad-0.622673 \quad-0.000073$

$\begin{array}{llll}\text { C } & -3.805383 & 0.011417 & 0.000114\end{array}$

H $\quad-2.412975 \quad-1.631614 \quad 0.000163$

H $\quad-3.927408 \quad 0.644016 \quad-0.891310$

$\begin{array}{llll}\text { H } & -4.571278 & -0.774406 & -0.000424\end{array}$

$\begin{array}{llll}H & -3.927709 & 0.643093 & 0.892160\end{array}$

\begin{abstract}
ALANINE
alanine_1 -494.53336002632 a.u.

$\begin{array}{llll}\text { C } & -3.077696 & -0.614096 & 0.770471\end{array}$

$\begin{array}{llll}\text { C } & -1.837634 & -0.380064 & -0.068647\end{array}$

O $\quad \begin{array}{llll}-1.529867 & -1.134105 & -1.009779\end{array}$
\end{abstract}

URL: http://mc.manuscriptcentral.com/tandf/tmph 


$$
\begin{array}{llll}
\mathrm{H} & -3.014507 & -1.617365 & 1.215958 \\
\mathrm{H} & -3.206763 & 0.133018 & 1.566818 \\
\mathrm{H} & -3.954515 & -0.593649 & 0.106906 \\
\mathrm{~N} & -1.085637 & 0.711655 & 0.257557 \\
\mathrm{C} & 0.134480 & 1.069664 & -0.474965 \\
\mathrm{C} & 1.315236 & 0.235479 & 0.061169 \\
\mathrm{O} & 2.168922 & 0.706249 & 0.827271 \\
\mathrm{C} & 0.403704 & 2.563351 & -0.339710 \\
\mathrm{H} & -1.323813 & 1.247033 & 1.085193 \\
\mathrm{H} & -0.048397 & 0.794167 & -1.526080 \\
\mathrm{H} & 1.304426 & 2.833869 & -0.908633 \\
\mathrm{H} & -0.452033 & 3.137886 & -0.725363 \\
\mathrm{H} & 0.588562 & 2.826041 & 0.712895 \\
\mathrm{~N} & 1.317422 & -1.054402 & -0.372498 \\
\mathrm{C} & 2.279406 & -2.008300 & 0.166327 \\
\mathrm{H} & 0.475135 & -1.379089 & -0.847767 \\
\mathrm{H} & 3.295361 & -1.603282 & 0.062520 \\
\mathrm{H} & 2.097251 & -2.207952 & 1.235029 \\
\mathrm{H} & 2.199387 & -2.944809 & -0.400683
\end{array}
$$

alanine_2 -494.53065171909 a.u.

C $\quad-3.366859 \quad-1.142124 \quad-0.000615$

$\begin{array}{llll}\text { C } & -2.312419 & -0.081812 & -0.258627\end{array}$

O $\quad-2.578516 \quad 1.000523 \quad-0.803466$

$\begin{array}{llll}H & -3.848821 & -1.395499 & -0.955887\end{array}$

H $\quad-2.955258 \quad-2.053815 \quad 0.456102$

H $\quad-4.134449 \quad-0.716837 \quad 0.662522$

$\begin{array}{llll}N & -1.045153 & -0.392395 & 0.146127\end{array}$ 


$$
\begin{array}{llll}
\mathrm{C} & 0.048393 & 0.545547 & -0.030045 \\
\mathrm{C} & 1.345438 & -0.255900 & 0.070600 \\
\mathrm{O} & 1.409581 & -1.318680 & 0.708184 \\
\mathrm{C} & 0.026939 & 1.650336 & 1.041756 \\
\mathrm{H} & -0.818215 & -1.277259 & 0.590800 \\
\mathrm{H} & -0.052176 & 1.003826 & -1.028869 \\
\mathrm{H} & 0.839074 & 2.375615 & 0.874530 \\
\mathrm{H} & -0.936851 & 2.176524 & 0.993300 \\
\mathrm{H} & 0.150928 & 1.200185 & 2.038820 \\
\mathrm{~N} & 2.431426 & 0.303145 & -0.528727 \\
\mathrm{C} & 3.738664 & -0.341795 & -0.453976 \\
\mathrm{H} & 2.301697 & 1.120310 & -1.111200 \\
\mathrm{H} & 3.996958 & -0.535949 & 0.596426 \\
\mathrm{H} & 3.737782 & -1.301254 & -0.993345 \\
\mathrm{H} & 4.485960 & 0.328646 & -0.897293
\end{array}
$$

alanine_3 $\quad-494.52972984894$ a.u.

$\begin{array}{llll}\text { C } & -3.146922 & -0.743545 & -0.625882\end{array}$

$\begin{array}{llll}\text { C } & -1.820406 & -0.349990 & -0.003653\end{array}$

$\begin{array}{llll}\text { O } & -1.408225 & -0.898737 & 1.035189\end{array}$

H $\quad-3.136531 \quad-1.826777 \quad-0.812937$

$\begin{array}{llll}H & -3.943901 & -0.534397 & 0.102892\end{array}$

H $\quad-3.364878 \quad-0.210896 \quad-1.562710$

$\begin{array}{llll}N & -1.127197 & 0.629874 & -0.651936\end{array}$

$\begin{array}{llll}\text { C } & 0.129782 & 1.254654 & -0.203070\end{array}$

$\begin{array}{llll}\text { C } & 1.371000 & 0.356162 & -0.357228\end{array}$

$\begin{array}{llll}O & 2.382815 & 0.779386 & -0.940581\end{array}$

URL: http://mc.manuscriptcentral.com/tandf/tmph 


$$
\begin{array}{llll}
\mathrm{C} & 0.032758 & 1.824145 & 1.220232 \\
\mathrm{H} & -1.515910 & 0.967340 & -1.523689 \\
\mathrm{H} & 0.312087 & 2.080949 & -0.905353 \\
\mathrm{H} & 0.977631 & 2.335378 & 1.461016 \\
\mathrm{H} & -0.788844 & 2.554629 & 1.278311 \\
\mathrm{H} & -0.143915 & 1.028890 & 1.955771 \\
\mathrm{~N} & 1.294828 & -0.871052 & 0.213667 \\
\mathrm{C} & 2.436246 & -1.775408 & 0.163178 \\
\mathrm{H} & 0.435499 & -1.118684 & 0.706234 \\
\mathrm{H} & 2.729193 & -1.966991 & -0.880054 \\
\mathrm{H} & 3.302787 & -1.342201 & 0.686066 \\
\mathrm{H} & 2.151896 & -2.720297 & 0.644002
\end{array}
$$

alanine_4 -494.52842446812 a.u.

$\begin{array}{llll}\text { C } & 3.210523 & -0.662723 & -0.460988\end{array}$

$\begin{array}{llll}\text { C } & 1.902501 & -0.329339 & 0.234014\end{array}$

$\begin{array}{llll}\text { O } & 1.592619 & -0.795233 & 1.335459\end{array}$

$\begin{array}{llll}H & 3.389166 & -1.743558 & -0.378568\end{array}$

H $\quad 3.222393 \quad-0.364554 \quad-1.519893$

$\begin{array}{llll}H & 4.026069 & -0.143867 & 0.065737\end{array}$

$\begin{array}{llll}N & 1.063614 & 0.528567 & -0.445477\end{array}$

$\begin{array}{llll}\text { C } & -0.109890 & 1.105277 & 0.209381\end{array}$

C $\quad-1.337755 \quad 0.182224 \quad 0.216490$

$\begin{array}{llll}O & -2.351017 & 0.508207 & 0.848319\end{array}$

$\begin{array}{llll}\text { C } & -0.482221 & 2.429954 & -0.463158\end{array}$

H $\quad 1.409974 \quad 0.975421 \quad-1.287344$

$\begin{array}{llll}H & 0.136098 & 1.268574 & 1.271371\end{array}$

$\begin{array}{llll}H & -1.380114 & 2.839518 & 0.018698\end{array}$

URL: http://mc.manuscriptcentral.com/tandf/tmph 


$$
\begin{array}{llll}
\mathrm{H} & 0.339979 & 3.156370 & -0.369155 \\
\mathrm{H} & -0.702916 & 2.270943 & -1.532093 \\
\mathrm{~N} & -1.258725 & -0.945502 & -0.543219 \\
\mathrm{C} & -2.326397 & -1.937418 & -0.487791 \\
\mathrm{H} & -0.338347 & -1.214934 & -0.868032 \\
\mathrm{H} & -3.291321 & -1.432334 & -0.626834 \\
\mathrm{H} & -2.174863 & -2.666844 & -1.294361 \\
\mathrm{H} & -2.343712 & -2.457830 & 0.483431
\end{array}
$$

alanine_5 $\quad-494.52628258390$ a.u.

$\begin{array}{llll}\text { C } & -3.025482 & -1.075360 & -0.074147\end{array}$

$\begin{array}{llll}\text { C } & -1.673961 & -0.442820 & 0.205604\end{array}$

$\begin{array}{llll}\mathrm{O} & -1.111030 & -0.527053 & 1.302023\end{array}$

$\begin{array}{llll}\text { H } & -3.062202 & -2.059331 & 0.412476\end{array}$

$\begin{array}{llll}H & -3.806005 & -0.443439 & 0.377657\end{array}$

H $\quad-3.233647 \quad-1.183722 \quad-1.148990$

$\begin{array}{llll}N & -1.092705 & 0.222116 & -0.855711\end{array}$

$\begin{array}{llll}\text { C } & 0.054423 & 1.117537 & -0.645550\end{array}$

$\begin{array}{llll}\text { C } & 1.294468 & 0.361408 & -0.131136\end{array}$

$\begin{array}{llll}\mathrm{O} & 2.172619 & 0.945485 & 0.512583\end{array}$

C $\quad-0.280066 \quad 2.308181 \quad 0.258116$

H $\quad-1.670731 \quad 0.386489 \quad-1.672458$

H $\quad 0.331884 \quad 1.486839 \quad-1.648165$

$\begin{array}{llll}H & 0.603106 & 2.952842 & 0.360338\end{array}$

$\begin{array}{llll}H & -1.105078 & 2.888571 & -0.183820\end{array}$

H $\quad-0.572888 \quad 1.953842 \quad 1.255792$

N $\quad 1.406073 \quad-0.939392 \quad-0.533569$

$\begin{array}{llll}\text { C } & 2.470132 & -1.767548 & 0.024114\end{array}$

URL: http://mc.manuscriptcentral.com/tandf/tmph 
H $\quad 0.552975 \quad-1.387601 \quad-0.845018$

H $\quad 3.433815 \quad-1.263280 \quad-0.124568$

$\begin{array}{llll}H & 2.326646 & -1.934370 & 1.104246\end{array}$

H $2.478743 \quad-2.731755 \quad-0.501390$

alanine_6 -494.52646519902 a.u.

$\begin{array}{llll}\text { C } & 2.974570 & -1.116271 & -0.062307\end{array}$

$\begin{array}{llll}\text { C } & 1.699117 & -0.341278 & -0.322664\end{array}$

O $\quad 0.989887 \quad-0.535484 \quad-1.318870$

H $\quad 3.492270 \quad-1.280126 \quad-1.016614$

$\begin{array}{llll}\text { H } & 3.644318 & -0.603055 & 0.642957\end{array}$

$\begin{array}{llll}H & 2.699141 & -2.097859 & 0.354607\end{array}$

$\begin{array}{llll}N & 1.364186 & 0.601008 & 0.624654\end{array}$

$\begin{array}{llll}\text { C } & 0.009715 & 1.164399 & 0.603282\end{array}$

C $\quad-1.004825 \quad 0.000252 \quad 0.591930$

O $\quad-0.935240 \quad-0.881154 \quad 1.457888$

C $\quad-0.140797 \quad 2.221470 \quad-0.495396$

H $\quad 1.801116 \quad 0.496087 \quad 1.533801$

$\begin{array}{llll}H & -0.133757 & 1.649062 & 1.582859\end{array}$

H $\quad-1.141871 \quad 2.678966 \quad-0.455125$

$\begin{array}{llll}H & 0.604554 & 3.014913 & -0.338257\end{array}$

H $\quad 0.029587 \quad 1.782705 \quad-1.489211$

$\begin{array}{llll}N & -1.993273 & 0.042415 & -0.348935\end{array}$

C $\quad-2.865522 \quad-1.120675 \quad-0.496527$

H $\quad-1.785949 \quad 0.576737 \quad-1.184520$

H $\quad-3.318936 \quad-1.352210 \quad 0.475711$

H $\quad-3.655464 \quad-0.879325 \quad-1.219954$

H $\quad-2.302117 \quad-2.004139 \quad-0.838343$

URL: http://mc.manuscriptcentral.com/tandf/tmph 


$$
\begin{aligned}
& \text { alanine_7 -494.52311928750 a.u. } \\
& \begin{array}{llll}
\text { C } & -3.057032 & -1.512273 & -0.105575
\end{array} \\
& \text { C } \quad-2.243919 \quad-0.229609 \quad-0.088498 \\
& \begin{array}{llll}
O & -2.768991 & 0.886557 & -0.142497
\end{array} \\
& \text { H } \quad-3.803773 \quad-1.462314 \quad 0.699722 \\
& \text { H } \quad-3.596440 \quad-1.570359-1.062559 \\
& \text { H } \quad-2.442470 \quad-2.415128 \quad 0.023446 \\
& \begin{array}{llll}
N & -0.875702 & -0.395257 & 0.013556
\end{array} \\
& \begin{array}{llll}
\text { C } & 0.014347 & 0.758107 & -0.185154
\end{array} \\
& \begin{array}{llll}
\text { C } & 1.450466 & 0.285311 & -0.403506
\end{array} \\
& \begin{array}{llll}
\text { O } & 2.211983 & 0.847603 & -1.196767
\end{array} \\
& \text { C } \quad-0.021042 \quad 1.713028 \quad 1.019355 \\
& \text { H } \quad-0.513940 \quad-1.305681 \quad-0.254458 \\
& \text { H } \quad-0.282336 \quad 1.307966 \quad-1.092609 \\
& \begin{array}{llll}
H & 0.671677 & 2.549888 & 0.840346
\end{array} \\
& \text { H } \quad-1.037116 \quad 2.106567 \quad 1.151393 \\
& \text { H } \quad 0.285158 \quad 1.186088 \quad 1.937333 \\
& \begin{array}{llll}
\mathrm{N} & 1.840930 & -0.791555 & 0.357429
\end{array} \\
& \begin{array}{llll}
\text { C } & 3.247337 & -1.189335 & 0.368071
\end{array} \\
& \begin{array}{llll}
H & 1.265703 & -1.024883 & 1.159878
\end{array} \\
& \begin{array}{llll}
H & 3.583566 & -1.338467 & -0.666165
\end{array} \\
& \begin{array}{llll}
H & 3.885179 & -0.418690 & 0.830030
\end{array} \\
& \begin{array}{llll}
H & 3.343311 & -2.131957 & 0.922704
\end{array}
\end{aligned}
$$


4

$\begin{array}{llll}\text { C } & -1.985847 & 0.256377 & 0.058699\end{array}$

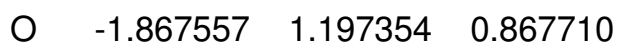

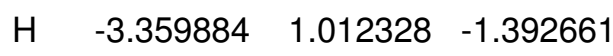

$\begin{array}{llll}\mathrm{H} & -3.155270 & -0.770492 & -1.482476\end{array}$

H $\quad-4.091405 \quad-0.023707 \quad-0.146631$

$\begin{array}{llll}N & -1.018521 & -0.691316 & -0.087241\end{array}$

C $\quad 0.211863 \quad-0.676130 \quad 0.700726$

$\begin{array}{llll}\text { C } & 1.227497 & 0.305061 & 0.085748\end{array}$

$\begin{array}{llll}\text { O } & 2.232960 & -0.090240 & -0.537938\end{array}$

$\begin{array}{llll}\text { C } & 0.787063 & -2.092439 & 0.726876\end{array}$

$\begin{array}{llll}\text { O } & 0.958958 & -2.592093 & -0.603138\end{array}$

H $\quad-1.077728 \quad-1.392311 \quad-0.820258$

$\begin{array}{llll}H & 1.637259 & -2.006543 & -0.988627\end{array}$

H $\quad-0.047199 \quad-0.342743 \quad 1.719776$

$\begin{array}{llll}H & 1.739484 & -2.088721 & 1.283149\end{array}$

$\begin{array}{llll}\text { H } & 0.080530 & -2.769095 & 1.230128\end{array}$

$\begin{array}{llll}N & 0.927618 & 1.611613 & 0.269056\end{array}$

$\begin{array}{llll}\text { C } & 1.728101 & 2.653416 & -0.364879\end{array}$

$\begin{array}{llll}H & 0.010191 & 1.818727 & 0.668264\end{array}$

$\begin{array}{llll}H & 2.787348 & 2.512916 & -0.109304\end{array}$

H $\quad 1.630566 \quad 2.620989-1.461786$

H $\quad 1.387550 \quad 3.628379 \quad 0.006040$

serine_2 $\quad-569.59916816$ a.u.

$\begin{array}{llll}\text { C } & 3.403344 & -0.419262 & 0.633643\end{array}$

$\begin{array}{llll}\text { C } & 2.106405 & -0.182277 & -0.112366\end{array}$

$\begin{array}{llll}\text { O } & 2.042449 & 0.571328 & -1.099767\end{array}$

$\begin{array}{llll}H & 3.809040 & 0.552463 & 0.949173\end{array}$

URL: http://mc.manuscriptcentral.com/tandf/tmph 


$$
\begin{array}{llll}
\mathrm{H} & 3.283166 & -1.070658 & 1.511169 \\
\mathrm{H} & 4.123005 & -0.876749 & -0.061139 \\
\mathrm{~N} & 1.010419 & -0.852105 & 0.353378 \\
\mathrm{C} & -0.297874 & -0.764813 & -0.296104 \\
\mathrm{C} & -1.053575 & 0.502802 & 0.146948 \\
\mathrm{O} & -2.080619 & 0.446149 & 0.851479 \\
\mathrm{C} & -1.109166 & -2.017738 & 0.050015 \\
\mathrm{O} & -2.400124 & -1.984675 & -0.542770 \\
\mathrm{H} & 1.087691 & -1.381118 & 1.215297 \\
\mathrm{H} & -2.856568 & -1.247352 & -0.101124 \\
\mathrm{H} & -0.124679 & -0.715953 & -1.383091 \\
\mathrm{H} & -0.592296 & -2.902798 & -0.350518 \\
\mathrm{H} & -1.177967 & -2.112014 & 1.150915 \\
\mathrm{~N} & -0.512085 & 1.662834 & -0.288678 \\
\mathrm{C} & -1.083991 & 2.945885 & 0.102637 \\
\mathrm{H} & 0.369481 & 1.604309 & -0.799509 \\
\mathrm{H} & -0.998178 & 3.103035 & 1.189175 \\
\mathrm{H} & -0.546122 & 3.742082 & -0.427296 \\
\mathrm{H} & -2.149417 & 2.979640 & -0.166131
\end{array}
$$$$
\text { serine_3 } \quad-569.59831430 \text { a.u. }
$$$$
\begin{array}{llll}
\text { C } & -3.557316 & -1.122781 & 0.178641
\end{array}
$$$$
\text { C } \quad-2.433710 \quad-0.157088-0.142845
$$$$
\begin{array}{llll}
O & -2.628431 & 0.925398 & -0.716508
\end{array}
$$$$
\text { H } \quad-0.983564 \quad-1.509072 \quad 0.507958
$$$$
\begin{array}{llll}
N & -1.180561 & -0.540859 & 0.260039
\end{array}
$$$$
\begin{array}{llll}
\text { C } & -0.028838 & 0.232669 & -0.157873
\end{array}
$$$$
\begin{array}{llll}
\text { C } & 1.199094 & -0.684661 & -0.071571
\end{array}
$$ 
$\begin{array}{llll}\text { O } & 1.089033 & -1.898208 & 0.174300\end{array}$

$\begin{array}{llll}\text { C } & 0.119537 & 1.494866 & 0.714196\end{array}$

$\begin{array}{llll}\mathrm{O} & 1.056141 & 2.427952 & 0.136415\end{array}$

$\begin{array}{llll}H & 0.591568 & 2.896329 & -0.572500\end{array}$

$\begin{array}{llll}H & 2.389038 & 0.943388 & -0.340747\end{array}$

$\begin{array}{llll}N & 2.390135 & -0.072505 & -0.282339\end{array}$

$\begin{array}{llll}\text { C } & 3.636388 & -0.821017 & -0.162544\end{array}$

$\begin{array}{llll}H & -3.210822 & -2.012232 & 0.723865\end{array}$

H $\quad-4.312078 \quad-0.595699 \quad 0.779833$

H $\quad-4.032047 \quad-1.431511 \quad-0.764340$

H $\quad-0.170729 \quad 0.562056 \quad-1.204575$

$\begin{array}{llll}H & -0.871779 & 1.955437 & 0.835001\end{array}$

$\begin{array}{llll}H & 0.520540 & 1.228519 & 1.703497\end{array}$

$\begin{array}{llll}\text { H } & 3.801965 & -1.159622 & 0.872260\end{array}$

$\begin{array}{llll}H & 3.601839 & -1.706220 & -0.812792\end{array}$

H $\quad 4.464182 \quad-0.170877 \quad-0.473035$

serine_4 $\quad-569.59692849$ a.u.

$\begin{array}{llll}\text { C } & -3.174638 & -1.646694 & 0.047929\end{array}$

$\begin{array}{llll}\text { C } & -2.233766 & -0.480919 & -0.176863\end{array}$

$\begin{array}{llll}\text { O } & -2.656332 & 0.638924 & -0.526217\end{array}$

$\begin{array}{llll}H & -3.949644 & -1.337356 & 0.763484\end{array}$

H $\quad-3.669700 \quad-1.881570 \quad-0.905924$

$\begin{array}{llll}H & -2.661629 & -2.543893 & 0.422001\end{array}$

$\begin{array}{llll}N & -0.912615 & -0.733646 & 0.010917\end{array}$

C $\quad 0.125363 \quad 0.262036 \quad-0.202379$

C $\quad \begin{array}{llll}1.458378 & -0.462779 & 0.015664\end{array}$

O $\quad 1.523889 \quad-1.568255 \quad 0.576779$

URL: http://mc.manuscriptcentral.com/tandf/tmph 


$$
\begin{array}{llll}
\mathrm{C} & -0.006407 & 1.500420 & 0.726896 \\
\mathrm{O} & -0.767689 & 2.543910 & 0.138608 \\
\mathrm{H} & -0.585382 & -1.634627 & 0.353596 \\
\mathrm{H} & -1.597890 & 2.118643 & -0.160038 \\
\mathrm{H} & 0.074586 & 0.636017 & -1.241575 \\
\mathrm{H} & -0.435364 & 1.157193 & 1.687321 \\
\mathrm{H} & 0.990210 & 1.923416 & 0.928086 \\
\mathrm{~N} & 2.558641 & 0.195766 & -0.431165 \\
\mathrm{C} & 3.892652 & -0.365129 & -0.237063 \\
\mathrm{H} & 2.444014 & 1.087307 & -0.897040 \\
\mathrm{H} & 4.100016 & -0.501581 & 0.834369 \\
\mathrm{H} & 3.972918 & -1.343348 & -0.733121 \\
\mathrm{H} & 4.627244 & 0.326712 & -0.667878
\end{array}
$$$$
\text { serine_5 } \quad-569.59791795 \text { a.u. }
$$$$
\text { C } \quad 3.088244 \quad-1.111930-0.434288
$$$$
\text { C } \quad \begin{array}{llll}
1.875631 & -0.213567 & -0.331124
\end{array}
$$$$
\begin{array}{llll}
\text { O } & 1.385296 & 0.338289 & -1.332409
\end{array}
$$$$
\text { H } \quad 3.757186 \quad-0.722215 \quad-1.212194
$$$$
\text { H } \quad 3.629090 \quad-1.197573 \quad 0.519061
$$$$
\begin{array}{llll}
H & 2.743993 & -2.113056 & -0.738924
\end{array}
$$$$
\text { N } \quad 1.357256 \quad-0.046132 \quad 0.925659
$$$$
\begin{array}{llll}
\text { C } & -0.018867 & 0.434955 & 1.117169
\end{array}
$$$$
\text { C } \quad-0.998438 \quad-0.599732 \quad 0.514265
$$$$
\text { O } \quad-0.834122 \quad-1.804691 \quad 0.759098
$$$$
\begin{array}{llll}
\text { C } & -0.226784 & 1.913919 & 0.734464
\end{array}
$$$$
\text { O } \quad-0.480536 \quad 2.163843 \quad-0.649908
$$$$
\begin{array}{llll}
H & 1.669480 & -0.727407 & 1.610360
\end{array}
$$ 
H $\quad 0.197587 \quad 1.637982 \quad-1.130116$

$\begin{array}{llll}H & -0.187546 & 0.383171 & 2.206538\end{array}$

$\begin{array}{llll}H & -1.109325 & 2.287871 & 1.276608\end{array}$

$\begin{array}{llll}H & 0.656244 & 2.477611 & 1.085594\end{array}$

$\begin{array}{llll}N & -2.039996 & -0.103097 & -0.200370\end{array}$

C $\quad-2.981463 \quad-1.025163 \quad-0.828065$

$\begin{array}{llll}H & -1.894941 & 0.827203 & -0.596593\end{array}$

$\begin{array}{llll}\text { H } & -3.360123 & -1.727187 & -0.073285\end{array}$

$\begin{array}{llll}H & -3.816429 & -0.444928 & -1.241756\end{array}$

H $\quad-2.501064 \quad-1.607291 \quad-1.631095$

serine_6 -569.59679809 a.u.

C $\quad-3.548102 \quad-1.130719 \quad 0.187665$

$\begin{array}{llll}\text { C } & -2.441719 & -0.149226 & -0.149826\end{array}$

$\begin{array}{llll}O & -2.655824 & 0.915859 & -0.745891\end{array}$

$\begin{array}{llll}H & -4.333516 & -0.597829 & 0.742387\end{array}$

H $\quad-3.987290 \quad-1.495995 \quad-0.752547$

$\begin{array}{llll}H & -3.193592 & -1.984968 & 0.781955\end{array}$

$\begin{array}{llll}N & -1.184145 & -0.506323 & 0.265965\end{array}$

C $\quad-0.028681 \quad 0.255104 \quad-0.167871$

$\begin{array}{llll}\text { C } & 1.189917 & -0.675612 & -0.053277\end{array}$

$\begin{array}{llll}\text { O } & 1.061820 & -1.878215 & 0.236689\end{array}$

$\begin{array}{llll}\text { C } & 0.104818 & 1.529231 & 0.677308\end{array}$

$\begin{array}{llll}\text { O } & 1.093120 & 2.370246 & 0.042464\end{array}$

H $\quad-0.980022 \quad-1.466725 \quad 0.538188$

$\begin{array}{llll}H & 1.091608 & 3.228207 & 0.487255\end{array}$

H $\quad-0.159477 \quad 0.569916 \quad-1.219381$

H $\quad-0.876199 \quad 2.027139 \quad 0.691250$

URL: http://mc.manuscriptcentral.com/tandf/tmph 


$$
\begin{array}{llll}
\mathrm{H} & 0.411111 & 1.267125 & 1.705749 \\
\mathrm{~N} & 2.390165 & -0.098615 & -0.302121 \\
\mathrm{C} & 3.619476 & -0.873990 & -0.181428 \\
\mathrm{H} & 2.413729 & 0.913811 & -0.392797 \\
\mathrm{H} & 3.799420 & -1.180943 & 0.860923 \\
\mathrm{H} & 3.548652 & -1.780271 & -0.798854 \\
\mathrm{H} & 4.456259 & -0.256752 & -0.532558
\end{array}
$$$$
\text { serine_7 } \quad-569.59554335 \text { a.u. }
$$$$
\begin{array}{llll}
\text { C } & -2.578215 & -1.968971 & 0.658379
\end{array}
$$$$
\begin{array}{llll}
\text { C } & -1.386441 & -1.325637 & -0.020786
\end{array}
$$

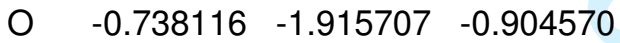$$
\begin{array}{llll}
H & -2.279656 & -2.960008 & 1.027762
\end{array}
$$$$
\begin{array}{llll}
H & -2.970861 & -1.368976 & 1.491645
\end{array}
$$$$
\begin{array}{llll}
H & -3.369346 & -2.110817 & -0.093034
\end{array}
$$$$
\begin{array}{llll}
N & -1.059087 & -0.068630 & 0.402843
\end{array}
$$$$
\begin{array}{llll}
\text { C } & -0.107240 & 0.763369 & -0.341706
\end{array}
$$$$
\begin{array}{llll}
\text { C } & 1.348233 & 0.457994 & 0.067169
\end{array}
$$$$
\begin{array}{llll}
O & 2.039414 & 1.278418 & 0.693580
\end{array}
$$$$
\begin{array}{llll}
\text { C } & -0.409066 & 2.229341 & -0.060532
\end{array}
$$$$
\begin{array}{llll}
O & -1.801696 & 2.434332 & -0.367993
\end{array}
$$$$
\begin{array}{llll}
H & -1.749133 & 0.429849 & 0.956072
\end{array}
$$$$
\text { H } \quad-2.005095 \quad 3.365983 \quad-0.209749
$$$$
\text { H } \quad-0.237469 \quad 0.545986-1.416420
$$$$
\begin{array}{llll}
H & -0.194041 & 2.455989 & 0.996481
\end{array}
$$$$
\begin{array}{llll}
H & 0.243305 & 2.854314 & -0.689849
\end{array}
$$$$
\text { N } \quad 1.795515 \quad-0.759357 \quad-0.325855
$$$$
\begin{array}{llll}
\text { C } & 3.119521 & -1.219929 & 0.072559
\end{array}
$$ 
H $\quad 1.107021 \quad-1.396403 \quad-0.730586$

H $\quad 3.189648 \quad-1.337199 \quad 1.165826$

$\begin{array}{llll}\text { H } & 3.313127 & -2.184834 & -0.413235\end{array}$

H $\quad 3.879943 \quad-0.491322 \quad-0.242465$ 
H $\quad 3.357616 \quad-2.113787 \quad-0.500982$

$$
\begin{aligned}
& \text { serine_9 } \quad-569.59467924 \text { a.u. } \\
& \begin{array}{llll}
\text { C } & -3.155250 & -1.068773 & -0.408166
\end{array} \\
& \begin{array}{llll}
\text { C } & -1.885407 & -0.355699 & 0.006988
\end{array} \\
& \text { O } \quad-1.547512 \quad-0.297165 \quad 1.208270 \\
& \text { H } \quad-1.468706 \quad 0.013290 \quad-1.939512 \\
& \begin{array}{llll}
N & -1.146907 & 0.189103 & -0.995155
\end{array} \\
& \text { C } \quad 0.151226 \quad 0.874566 \quad-0.867016 \\
& \begin{array}{llll}
\text { C } & 1.356257 & -0.044947 & -0.604006
\end{array} \\
& \begin{array}{llll}
\text { O } & 2.448657 & 0.221768 & -1.122489
\end{array} \\
& \begin{array}{llll}
\text { C } & 0.156340 & 2.047443 & 0.141475
\end{array} \\
& \begin{array}{llll}
\mathrm{O} & 0.367623 & 1.672551 & 1.494683
\end{array} \\
& \begin{array}{llll}
H & -0.333942 & 1.028476 & 1.706690
\end{array} \\
& \begin{array}{llll}
H & 0.319510 & -1.082550 & 0.802330
\end{array} \\
& \begin{array}{llll}
N & 1.142857 & -1.119316 & 0.205589
\end{array} \\
& \begin{array}{llll}
\text { C } & 2.289947 & -1.909321 & 0.645627
\end{array} \\
& \text { H } \quad-3.386474 \quad-0.958174 \quad-1.476979 \\
& \text { H } \quad-3.045221 \quad-2.136856 \quad-0.168207 \\
& \begin{array}{llll}
H & -3.986836 & -0.672357 & 0.191239
\end{array} \\
& \begin{array}{llll}
H & 0.342732 & 1.314543 & -1.857924
\end{array} \\
& \begin{array}{llll}
H & 1.001482 & 2.697899 & -0.128309
\end{array} \\
& \begin{array}{llll}
H & -0.784035 & 2.616598 & 0.010741
\end{array} \\
& \begin{array}{llll}
H & 2.863748 & -2.233962 & -0.232353
\end{array} \\
& \begin{array}{llll}
H & 2.956294 & -1.322523 & 1.298373
\end{array} \\
& \begin{array}{llll}
H & 1.920975 & -2.789747 & 1.187754
\end{array}
\end{aligned}
$$$$
\text { serine_10 } \quad-569.59451888 \text { a.u. }
$$ 
$\begin{array}{llll}\text { C } & -3.413970 & -1.128540 & 0.444231\end{array}$

C $\quad-2.370408 \quad-0.276987-0.253336$

O $\quad \begin{array}{llll}-2.644249 & 0.467487 & -1.204042\end{array}$

H $\quad-4.248313 \quad-0.481482 \quad 0.750243$

H $\quad-3.803155 \quad-1.862299-0.277234$

H $\quad-3.015751-1.656031 \quad 1.322765$

$\begin{array}{llll}N & -1.094650 & -0.367448 & 0.250708\end{array}$

$\begin{array}{llll}\text { C } & 0.010525 & 0.254244 & -0.459983\end{array}$

C $\quad 1.281189-0.503850-0.052210$

$\begin{array}{llll}\text { O } & 1.251642 & -1.429183 & 0.773531\end{array}$

C $\quad 0.102851 \quad 1.743596 \quad-0.090808$

$\begin{array}{llll}\text { O } & 0.451137 & 1.918807 & 1.283630\end{array}$

H $\quad-0.829078 \quad-1.144709 \quad 0.854308$

H $\quad-0.232832 \quad 1.467976 \quad 1.802495$

H $\quad-0.1568690 .169165 \quad-1.549947$

$\begin{array}{llll}\text { H } & 0.893245 & 2.244772 & -0.670942\end{array}$

H $\quad-0.860455 \quad 2.219732 \quad-0.337249$

N $\quad 2.427563 \quad-0.107840 \quad-0.664507$

C $\quad 3.711799-0.676974 \quad-0.265732$

H $\quad 2.409409 \quad 0.720129 \quad-1.245555$

$\begin{array}{llll}H & 3.970144 & -0.389346 & 0.765067\end{array}$

H $\quad 3.658896-1.772699 \quad-0.319586$

H $\quad 4.484211-0.314022 \quad-0.955703$

serine_11 -569.59403494 a.u.

C $\quad-2.162173 \quad-2.277649 \quad 0.819050$

$\begin{array}{llll}\text { C } & -1.291874 & -1.392197 & -0.049447\end{array}$

O $\quad-0.810226 \quad-1.788123 \quad-1.121775$

URL: http://mc.manuscriptcentral.com/tandf/tmph 


$$
\begin{array}{llll}
\mathrm{H} & -1.272204 & 0.070973 & 1.392330 \\
\mathrm{~N} & -1.089312 & -0.111164 & 0.408908 \\
\mathrm{C} & -0.174687 & 0.804174 & -0.285862 \\
\mathrm{C} & 1.274292 & 0.521710 & 0.135680 \\
\mathrm{O} & 1.865091 & 1.211972 & 0.980818 \\
\mathrm{C} & -0.581341 & 2.242469 & 0.021452 \\
\mathrm{O} & -1.871375 & 2.546855 & -0.519878 \\
\mathrm{H} & -2.475929 & 1.846179 & -0.233339 \\
\mathrm{H} & 1.206309 & -1.143375 & -1.035107 \\
\mathrm{~N} & 1.828920 & -0.555408 & -0.483636 \\
\mathrm{C} & 3.148367 & -1.035076 & -0.088191 \\
\mathrm{H} & -2.531450 & -1.767649 & 1.720295 \\
\mathrm{H} & -3.017722 & -2.619253 & 0.218562 \\
\mathrm{H} & -1.579629 & -3.163746 & 1.110354 \\
\mathrm{H} & -0.301453 & 0.607596 & -1.361541 \\
\mathrm{H} & -0.544673 & 2.418441 & 1.111307 \\
\mathrm{H} & 0.128591 & 2.933270 & -0.452541 \\
\mathrm{H} & 3.865962 & -0.203954 & -0.121820 \\
\mathrm{H} & 3.140069 & -1.441063 & 0.936305 \\
\mathrm{H} & 3.461444 & -1.817639 & -0.791117
\end{array}
$$


$\begin{array}{llll}\text { C } & -1.195580 & -0.596268 & -0.068735\end{array}$

O $\quad-1.177063 \quad-1.570516 \quad-0.837682$

$\begin{array}{llll}\text { C } & -0.044298 & 1.561211 & -0.705116\end{array}$

O $\quad-1.108705 \quad 2.430831-0.302740$

$\begin{array}{llll}H & -1.935637 & 2.052821 & -0.633435\end{array}$

$\begin{array}{llll}H & -2.235732 & 0.528577 & 1.284536\end{array}$

$\begin{array}{llll}N & -2.326655 & -0.187359 & 0.572996\end{array}$

$\begin{array}{llll}\text { C } & -3.559762 & -0.964472 & 0.471837\end{array}$

H $\quad 3.224313 \quad-1.911363 \quad-0.858042$

H $\quad 4.337827 \quad-0.514232 \quad-0.681064$

H $\quad 4.011551 \quad-1.562463 \quad 0.717352$

H $\quad 0.131155 \quad 0.580052 \quad 1.210266$

$\begin{array}{llll}H & 0.889102 & 2.119907 & -0.554291\end{array}$

H $\quad-0.129208 \quad 1.274874 \quad-1.768592$

H $\quad-3.786488 \quad-1.151576 \quad-0.586365$

H $\quad-3.464373 \quad-1.935533 \quad 0.982126$

$\begin{array}{llll}H & -4.375989 & -0.388267 & 0.926027\end{array}$

serine_13 -569.59381212 a.u.

$\begin{array}{llll}\text { C } & 3.382938 & 0.237140 & -0.014381\end{array}$

$\begin{array}{llll}\text { C } & 1.890335 & 0.095646 & 0.219638\end{array}$

$\begin{array}{llll}\text { O } & 1.352324 & 0.370460 & 1.296531\end{array}$

H $\quad 3.720863 \quad 1.183113 \quad 0.430315$

$\begin{array}{llll}H & 3.895813 & -0.584744 & 0.508488\end{array}$

$\begin{array}{llll}H & 3.656027 & 0.208801 & -1.079461\end{array}$

N $\quad 1.151005 \quad-0.338232 \quad-0.865183$

$\begin{array}{llll}\text { C } & -0.241930 & -0.760868 & -0.699821\end{array}$

$\begin{array}{llll}\text { C } & -1.129027 & 0.369559 & -0.152318\end{array}$

O $\quad-2.125359 \quad 0.116973 \quad 0.544396$

URL: http://mc.manuscriptcentral.com/tandf/tmph 


$$
\begin{array}{llll}
\mathrm{C} & -0.380351 & -2.022674 & 0.173565 \\
\mathrm{O} & -1.684566 & -2.583478 & 0.057672 \\
\mathrm{H} & 1.658848 & -0.697897 & -1.665794 \\
\mathrm{H} & -2.276722 & -1.885360 & 0.391749 \\
\mathrm{H} & -0.627739 & -1.008197 & -1.703433 \\
\mathrm{H} & 0.326889 & -2.782289 & -0.195331 \\
\mathrm{H} & -0.128780 & -1.774741 & 1.217734 \\
\mathrm{~N} & -0.813634 & 1.628493 & -0.556283 \\
\mathrm{C} & -1.514091 & 2.770626 & 0.023106 \\
\mathrm{H} & 0.117573 & 1.760685 & -0.932985 \\
\mathrm{H} & -2.596307 & 2.621182 & -0.083444 \\
\mathrm{H} & -1.278527 & 2.881797 & 1.093728 \\
\mathrm{H} & -1.215958 & 3.677605 & -0.518823
\end{array}
$$$$
\text { serine_14 -569.59297755 a.u. }
$$$$
\begin{array}{llll}
\text { C } & 2.710020 & -1.743500 & 0.582667
\end{array}
$$$$
\begin{array}{llll}
\text { C } & 1.568943 & -0.910238 & 0.032302
\end{array}
$$$$
\text { O } \quad 1.183610 \quad-1.035156 \quad-1.147255
$$$$
\begin{array}{llll}
H & 2.410354 & -2.801531 & 0.555465
\end{array}
$$$$
\text { H } 3.579392 \quad-1.623814 \quad-0.079653
$$$$
\text { H } \quad 2.991667 \quad-1.471666 \quad 1.609913
$$$$
N \quad 0.992236 \quad-0.035089 \quad 0.901421
$$$$
\begin{array}{llll}
\text { C } & -0.081507 & 0.916785 & 0.584865
\end{array}
$$$$
\begin{array}{llll}
\text { C } & -1.476793 & 0.280399 & 0.462397
\end{array}
$$$$
\text { O } \quad-2.445022 \quad 0.749098 \quad 1.081205
$$$$
\begin{array}{llll}
\text { C } & 0.215602 & 1.769781 & -0.665891
\end{array}
$$$$
\text { O } \quad 1.492505 \quad 2.406519 \quad-0.583293
$$$$
\text { H } \quad 1.366779 \quad 0.000341 \quad 1.841272
$$ 
$\begin{array}{llll}H & 2.154317 & 1.752617 & -0.848773\end{array}$

H $\quad-0.143538 \quad 1.594679 \quad 1.447742$

H $\quad 0.129995 \quad 1.165622 \quad-1.580086$

H $\quad-0.536298 \quad 2.572543 \quad-0.696810$

N $\quad-1.567702 \quad-0.773814 \quad-0.388162$

C $\quad-2.852578-1.423694 \quad-0.616166$

H $\quad-0.732849 \quad-1.048347 \quad-0.906220$

$\begin{array}{llll}H & -3.276018 & -1.775578 & 0.336218\end{array}$

H $\quad-3.570990 \quad-0.724919-1.071756$

H $\quad-2.695412 \quad-2.278502 \quad-1.286432$

serine_15 -569.59288500 a.u.

C $\quad 2.877630 \quad-1.628808 \quad-0.386827$

$\begin{array}{llll}\text { C } & 1.591186 & -1.102213 & 0.221581\end{array}$

O $\quad 1.013025-1.660412 \quad 1.159156$

H $\quad 2.798082 \quad-2.718977 \quad-0.495857$

$\begin{array}{llll}\text { H } & 3.104758 & -1.172077 & -1.361041\end{array}$

H $\quad 3.703814 \quad-1.417922 \quad 0.309742$

N $\quad \begin{array}{lll}1.079412 & 0.046043 & -0.356683\end{array}$

$\begin{array}{llll}\text { C } & 0.044774 & 0.792243 & 0.351608\end{array}$

$\begin{array}{llll}\text { C } & -1.344670 & 0.147036 & 0.310979\end{array}$

O $\quad-2.226707 \quad 0.537149 \quad 1.086958$

$\begin{array}{llll}\text { C } & -0.074601 & 2.183137 & -0.285477\end{array}$

$\begin{array}{llll}\text { O } & 1.193560 & 2.855321 & -0.343599\end{array}$

H $\quad 1.721996 \quad 0.612673 \quad-0.905612$

H $\quad 1.428448 \quad 3.121504 \quad 0.557102$

$\begin{array}{llll}H & 0.300255 & 0.879985 & 1.422751\end{array}$

H $\quad-0.400772 \quad 2.082229 \quad-1.332059$

URL: http://mc.manuscriptcentral.com/tandf/tmph 


$$
\begin{array}{llll}
\mathrm{H} & -0.827717 & 2.765123 & 0.267578 \\
\mathrm{~N} & -1.552201 & -0.766146 & -0.676625 \\
\mathrm{C} & -2.803536 & -1.514011 & -0.726835 \\
\mathrm{H} & -0.725431 & -1.139283 & -1.128419 \\
\mathrm{H} & -3.642950 & -0.811279 & -0.644701 \\
\mathrm{H} & -2.861810 & -2.043230 & -1.687026 \\
\mathrm{H} & -2.872872 & -2.238795 & 0.100401
\end{array}
$$$$
\text { serine_16 -569.59284583 a.u. }
$$$$
\begin{array}{llll}
\text { C } & 2.833451 & -1.624179 & 0.532462
\end{array}
$$$$
\begin{array}{llll}
\text { C } & 1.551548 & -0.976733 & 0.047861
\end{array}
$$$$
\begin{array}{llll}
\text { O } & 1.051629 & -1.272835 & -1.054246
\end{array}
$$

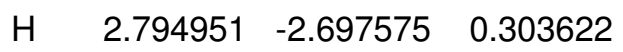$$
\text { H } \quad 3.672626 \quad-1.183303 \quad-0.027402
$$$$
\begin{array}{llll}
H & 3.007413 & -1.479075 & 1.608471
\end{array}
$$$$
\begin{array}{llll}
N & 0.991913 & -0.062690 & 0.891991
\end{array}
$$$$
\text { C } \quad-0.056597 \quad 0.901191 \quad 0.528820
$$$$
\begin{array}{llll}
\text { C } & -1.480036 & 0.323892 & 0.431302
\end{array}
$$$$
\begin{array}{llll}
O & -2.428384 & 0.907313 & 0.981551
\end{array}
$$$$
\begin{array}{llll}
\text { C } & 0.281692 & 1.682609 & -0.743519
\end{array}
$$$$
\begin{array}{llll}
\text { O } & 1.536132 & 2.339328 & -0.498572
\end{array}
$$$$
\begin{array}{llll}
H & 1.527331 & 0.166995 & 1.720278
\end{array}
$$$$
\begin{array}{llll}
H & 1.807916 & 2.777071 & -1.316506
\end{array}
$$$$
\text { H } \quad-0.103931 \quad 1.620703 \quad 1.358638
$$$$
\begin{array}{llll}
H & 0.351123 & 1.002793 & -1.606300
\end{array}
$$$$
\text { H } \quad-0.528409 \quad 2.414224 \quad-0.910700
$$$$
\text { N } \quad-1.618527 \quad-0.797653 \quad-0.315490
$$$$
\begin{array}{llll}
\text { C } & -2.933617 & -1.398891 & -0.495908
\end{array}
$$ 
$\begin{array}{llll}\mathrm{H} & -0.786057 & -1.170611 & -0.776691\end{array}$

H $\quad-3.613262 \quad-0.706446 \quad-1.016118$

H $\quad-2.817890 \quad-2.315522 \quad-1.088422$

$\begin{array}{llll}\text { H } & -3.379176 & -1.644629 & 0.479647\end{array}$

serine_17 -569.59174969 a.u.

$\begin{array}{llll}\text { C } & -3.448900 & -1.071357 & 0.480615\end{array}$

C $\quad-2.384893 \quad-0.274624 \quad-0.249959$

O $\quad-2.655105 \quad 0.488600 \quad-1.191900$

H $\quad-4.186382 \quad-0.370540 \quad 0.898143$

H $\quad-3.966702 \quad-1.712141 \quad-0.247913$

$\begin{array}{llll}H & -3.037078 & -1.692833 & 1.288667\end{array}$

$\begin{array}{llll}N & -1.106306 & -0.439816 & 0.196678\end{array}$

$\begin{array}{llll}\text { C } & -0.001341 & 0.221804 & -0.460635\end{array}$

C $\quad \begin{array}{llll}1.280803 & -0.517996 & -0.051132\end{array}$

$\begin{array}{llll}\mathrm{O} & 1.271131 & -1.429782 & 0.786136\end{array}$

$\begin{array}{llll}\text { C } & 0.075273 & 1.705852 & -0.083030\end{array}$

$\begin{array}{llll}\mathrm{O} & 0.504457 & 1.776174 & 1.282510\end{array}$

H $\quad-0.861258 \quad-1.116258 \quad 0.914522$

$\begin{array}{llll}H & 0.265269 & 2.644350 & 1.632642\end{array}$

$\begin{array}{llll}H & -0.142496 & 0.158568 & -1.554861\end{array}$

$\begin{array}{llll}H & 0.790222 & 2.228724 & -0.747485\end{array}$

$\begin{array}{llll}H & -0.923387 & 2.145971 & -0.229199\end{array}$

$\begin{array}{llll}N & 2.417214 & -0.113884 & -0.682781\end{array}$

$\begin{array}{llll}\text { C } & 3.714029 & -0.640974 & -0.268352\end{array}$

$\begin{array}{llll}H & 2.380826 & 0.713175 & -1.264710\end{array}$

H $\quad 3.687226 \quad-1.738576 \quad-0.294702$

H $\quad 4.478910 \quad-0.278157 \quad-0.966976$

URL: http://mc.manuscriptcentral.com/tandf/tmph 


$$
\begin{array}{llll}
H & 3.964800 & -0.322546 & 0.755580
\end{array}
$$

serine_18 -569.59263641 a.u.

$\begin{array}{llll}\text { C } & 3.487401 & 0.279265 & -0.331846\end{array}$

$\begin{array}{llll}\text { C } & 2.037430 & 0.144939 & 0.090241\end{array}$

$\begin{array}{llll}O & 1.636955 & 0.565601 & 1.190398\end{array}$

$\begin{array}{llll}H & 3.785510 & 1.331918 & -0.226821\end{array}$

$\begin{array}{llll}H & 4.102311 & -0.316027 & 0.359421\end{array}$

H $\quad 3.674825 \quad-0.055856 \quad-1.361964$

$\begin{array}{llll}N & 1.205718 & -0.453257 & -0.812332\end{array}$

C $\quad-0.193631 \quad-0.829873 \quad-0.587730$

$\begin{array}{llll}\text { C } & -1.215286 & 0.307070 & -0.460072\end{array}$

$\begin{array}{llll}O & -2.403616 & 0.068657 & -0.753363\end{array}$

$\begin{array}{llll}\text { C } & -0.383697 & -1.774612 & 0.624678\end{array}$

$\begin{array}{llll}O & -1.638795 & -2.447107 & 0.542666\end{array}$

$\begin{array}{llll}\mathrm{H} & 1.611641 & -0.738338 & -1.694840\end{array}$

H $\quad-2.300738 \quad-1.787772 \quad 0.266947$

H $\quad-0.504275 \quad-1.398101 \quad-1.477379$

$\begin{array}{llll}H & 0.397245 & -2.550019 & 0.594218\end{array}$

H $\quad-0.284086 \quad-1.208090 \quad 1.565712$

$\begin{array}{llll}N & -0.789080 & 1.496870 & 0.027489\end{array}$

$\begin{array}{llll}\text { C } & -1.767529 & 2.540618 & 0.321183\end{array}$

$\begin{array}{llll}H & 0.102545 & 1.486329 & 0.525217\end{array}$

H $\quad-2.391759 \quad 2.713694 \quad-0.565488$

$\begin{array}{llll}H & -2.426498 & 2.253755 & 1.156466\end{array}$

H $\quad-1.227683 \quad 3.461563 \quad 0.576080$

serine_19 -569.59210256 a.u. 
$\begin{array}{llll}\text { C } & 2.855595 & -1.620455 & 0.520731\end{array}$

$\begin{array}{llll}\text { C } & 1.565296 & -0.985291 & 0.042645\end{array}$

$\begin{array}{llll}\text { O } & 1.050542 & -1.301498 & -1.046182\end{array}$

H $\quad 1.546789 \quad 0.168114 \quad 1.710395$

$\begin{array}{llll}N & 1.016816 & -0.054245 & 0.877047\end{array}$

$\begin{array}{llll}\text { C } & -0.049383 & 0.891636 & 0.520447\end{array}$

$\begin{array}{llll}\text { C } & -1.469703 & 0.305710 & 0.432327\end{array}$

$\begin{array}{llll}\text { O } & -2.419302 & 0.908797 & 0.960657\end{array}$

$\begin{array}{llll}\text { C } & 0.274328 & 1.677979 & -0.762406\end{array}$

$\begin{array}{llll}O & 1.498104 & 2.413755 & -0.636724\end{array}$

$\begin{array}{llll}\text { H } & 1.320984 & 3.193485 & -0.092073\end{array}$

H $\quad-0.775791 \quad-1.207070 \quad-0.749464$

$\begin{array}{llll}N & -1.606705 & -0.833972 & -0.284939\end{array}$

C $\quad-2.924531 \quad-1.428224 \quad-0.470125$

$\begin{array}{llll}H & 3.041801 & -1.460968 & 1.592735\end{array}$

$\begin{array}{llll}H & 2.819557 & -2.696744 & 0.305831\end{array}$

$\begin{array}{llll}H & 3.685587 & -1.182294 & -0.054757\end{array}$

H $\quad-0.108733 \quad 1.606257 \quad 1.355552$

$\begin{array}{llll}H & 0.418369 & 0.988698 & -1.603787\end{array}$

H $\quad-0.579729 \quad 2.341294 \quad-0.984099$

H $\quad-3.391911 \quad-1.627813 \quad 0.505450$

H $\quad-3.585893 \quad-0.751788-1.033471$

H $\quad-2.806172 \quad-2.370209-1.020783$

serine_20 -569.59229424 a.u.

C $\quad 2.872406-1.637078 \quad-0.364256$

$\begin{array}{llll}\text { C } & 1.568505 & -1.121698 & 0.215685\end{array}$

O $\quad 0.960386 \quad-1.697748 \quad 1.122853$

URL: http://mc.manuscriptcentral.com/tandf/tmph 


$$
\begin{array}{llll}
\mathrm{H} & 2.831668 & -2.733293 & -0.413498 \\
\mathrm{H} & 3.082645 & -1.223658 & -1.361518 \\
\mathrm{H} & 3.690899 & -1.355929 & 0.316999 \\
\mathrm{~N} & 1.079689 & 0.042190 & -0.353055 \\
\mathrm{C} & 0.052021 & 0.791658 & 0.366616 \\
\mathrm{C} & -1.346294 & 0.165091 & 0.305826 \\
\mathrm{O} & -2.234303 & 0.565729 & 1.069395 \\
\mathrm{C} & -0.051378 & 2.189077 & -0.240773 \\
\mathrm{O} & 1.273634 & 2.753746 & -0.225730 \\
\mathrm{H} & 1.749461 & 0.615821 & -0.860539 \\
\mathrm{H} & 1.220259 & 3.654972 & -0.571624 \\
\mathrm{H} & 0.310784 & 0.864992 & 1.436259 \\
\mathrm{H} & -0.428986 & 2.115172 & -1.277003 \\
\mathrm{H} & -0.754901 & 2.780301 & 0.364946 \\
\mathrm{~N} & -1.552206 & -0.748038 & -0.682338 \\
\mathrm{C} & -2.807742 & -1.488244 & -0.737934 \\
\mathrm{H} & -0.723609 & -1.133184 & -1.120413 \\
\mathrm{H} & -3.643765 & -0.779842 & -0.671132 \\
\mathrm{H} & -2.859670 & -2.026560 & -1.693457 \\
\mathrm{H} & -2.890016 & -2.204513 & 0.095601
\end{array}
$$


$\begin{array}{llll}\text { C } & -1.632051 & 0.305941 & 0.125255\end{array}$

O $\quad \begin{array}{llll}-2.428933 & 1.249312 & 0.219811\end{array}$

$\begin{array}{llll}\text { C } & 0.269285 & 1.879012 & -0.382951\end{array}$

$\begin{array}{llll}\text { O } & 1.689991 & 2.049967 & -0.408276\end{array}$

$\begin{array}{llll}H & 2.024094 & 1.896661 & 0.489325\end{array}$

H $\quad-1.315843 \quad-1.702457 \quad-0.022314$

N $\quad-2.025758 \quad-0.982088-0.087774$

$\begin{array}{llll}\text { C } & -3.442926 & -1.327577 & -0.078888\end{array}$

$\begin{array}{llll}\text { H } & 3.442733 & -2.312719 & 0.016523\end{array}$

$\begin{array}{llll}H & 2.264528 & -2.307639 & -1.345426\end{array}$

H $\quad 3.477783 \quad-1.000374 \quad-1.191420$

H $\quad 0.121132 \quad 0.550406 \quad 1.319853$

H $\quad-0.055642 \quad 1.914664 \quad-1.435093$

$\begin{array}{llll}H & -0.239796 & 2.689360 & 0.160964\end{array}$

$\begin{array}{llll}H & -3.556041 & -2.362457 & -0.427078\end{array}$

$\begin{array}{llll}\text { H } & -3.876010 & -1.227017 & 0.929011\end{array}$

H $\quad-3.984633 \quad-0.651774 \quad-0.754406$

serine_22 -569.59147111 a.u.

$\begin{array}{llll}\text { C } & 3.360974 & -0.323472 & 0.619450\end{array}$

C $\quad 2.061561 \quad-0.225220 \quad-0.153356$

O $\quad 1.971459 \quad 0.410743 \quad-1.215804$

H $\quad 1.052193 \quad-1.219244 \quad 1.340311$

$\begin{array}{llll}N & 0.991240 & -0.900189 & 0.378506\end{array}$

C $\quad-0.336415 \quad-0.768235 \quad-0.222914$

$\begin{array}{llll}\text { C } & -0.981868 & 0.534850 & 0.277301\end{array}$

O $\quad-1.647952 \quad 0.576185 \quad 1.320128$

$\begin{array}{llll}\text { C } & -1.199365 & -1.979317 & 0.125655\end{array}$

URL: http://mc.manuscriptcentral.com/tandf/tmph 


$$
\begin{array}{llll}
\mathrm{O} & -2.440238 & -1.950687 & -0.583558 \\
\mathrm{H} & -2.252234 & -2.116208 & -1.518185 \\
\mathrm{H} & 0.056124 & 1.516819 & -1.161651 \\
\mathrm{~N} & -0.708906 & 1.621999 & -0.498122 \\
\mathrm{C} & -1.091118 & 2.954449 & -0.042484 \\
\mathrm{H} & 3.287858 & -0.975165 & 1.501817 \\
\mathrm{H} & 4.139139 & -0.708111 & -0.055237 \\
\mathrm{H} & 3.656861 & 0.687948 & 0.934709 \\
\mathrm{H} & -0.178689 & -0.720025 & -1.312451 \\
\mathrm{H} & -0.629879 & -2.902796 & -0.082513 \\
\mathrm{H} & -1.465681 & -1.945947 & 1.192111 \\
\mathrm{H} & -2.154975 & 2.949642 & 0.229192 \\
\mathrm{H} & -0.510241 & 3.264737 & 0.841687 \\
\mathrm{H} & -0.925576 & 3.667423 & -0.860526
\end{array}
$$

serine_23 -569.59133060 a.u.

$\begin{array}{llll}\text { C } & 3.415234 & -0.572591 & 0.244905\end{array}$

C $\quad 1.989986 \quad-0.223614 \quad-0.137416$

O $\quad 1.523968 \quad-0.537806-1.247116$

$\begin{array}{llll}H & 3.690331 & -0.237032 & 1.254926\end{array}$

$\begin{array}{llll}H & 3.535622 & -1.663313 & 0.175610\end{array}$

H $\quad 4.091889 \quad-0.110872 \quad-0.488724$

$\begin{array}{llll}N & 1.271611 & 0.457991 & 0.805346\end{array}$

$\begin{array}{llll}\text { C } & -0.123939 & 0.896566 & 0.655632\end{array}$

$\begin{array}{llll}\text { C } & -1.109567 & -0.288903 & 0.649246\end{array}$

$\begin{array}{llll}\text { O } & -1.793877 & -0.557454 & 1.645424\end{array}$

C $\quad-0.305181 \quad 1.837787 \quad-0.548814$

$\begin{array}{llll}O & -1.663877 & 2.273868 & -0.635402\end{array}$ 
H $\quad 1.707406 \quad 0.602875 \quad 1.707799$

H $\quad-2.186537 \quad 1.516338 \quad-0.939424$

H $\quad-0.375042 \quad 1.455697 \quad 1.568385$

$\begin{array}{llll}H & 0.300058 & 2.741847 & -0.383507\end{array}$

$\begin{array}{llll}\text { H } & 0.031171 & 1.358624 & -1.480021\end{array}$

$\begin{array}{llll}N & -1.200831 & -0.962312 & -0.537884\end{array}$

C $\quad-2.000812 \quad-2.183907-0.597426$

H $\quad-0.379297 \quad-0.880270-1.141845$

H $\quad-3.017674 \quad-1.969298 \quad-0.242921$

H $\quad-2.040026 \quad-2.525664 \quad-1.639894$

$\begin{array}{llll}\text { H } & -1.577403 & -2.979573 & 0.037362\end{array}$

serine_24 -569.59159155 a.u.

$\begin{array}{llll}\text { C } & -3.368232 & -0.697605 & -0.005877\end{array}$

$\begin{array}{llll}\text { C } & -2.044779 & -0.023753 & 0.287920\end{array}$

$\begin{array}{llll}\mathrm{O} & -1.582025 & 0.086522 & 1.426521\end{array}$

H $\quad-4.014268 \quad-0.622803 \quad 0.877715$

$\begin{array}{llll}H & -3.871624 & -0.258975 & -0.880328\end{array}$

H $\quad-3.165531 \quad-1.761470 \quad-0.209930$

$\begin{array}{llll}N & -1.378254 & 0.480070 & -0.820537\end{array}$

$\begin{array}{llll}\text { C } & 0.063347 & 0.678185 & -0.710789\end{array}$

$\begin{array}{llll}\text { C } & 0.752395 & -0.664955 & -0.371925\end{array}$

O $\quad 0.187176 \quad-1.747456 \quad-0.580849$

$\begin{array}{llll}\text { C } & 0.409001 & 1.829697 & 0.242811\end{array}$

$\begin{array}{llll}\text { O } & 1.749321 & 2.261712 & -0.109384\end{array}$

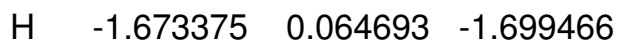

$\begin{array}{llll}H & 1.998720 & 2.967613 & 0.503024\end{array}$

$\begin{array}{llll}H & 0.422195 & 0.985451 & -1.708688\end{array}$

URL: http://mc.manuscriptcentral.com/tandf/tmph 


$$
\begin{array}{llll}
\mathrm{H} & -0.313497 & 2.645681 & 0.079739 \\
\mathrm{H} & 0.364080 & 1.498595 & 1.288988 \\
\mathrm{~N} & 2.012849 & -0.565238 & 0.121370 \\
\mathrm{C} & 2.786644 & -1.766373 & 0.409616 \\
\mathrm{H} & 2.429266 & 0.359853 & 0.179781 \\
\mathrm{H} & 2.243591 & -2.401946 & 1.123922 \\
\mathrm{H} & 2.963479 & -2.351768 & -0.506152 \\
\mathrm{H} & 3.748763 & -1.466145 & 0.844722
\end{array}
$$


$\begin{array}{llll}\text { C } & 3.189303 & -0.977159 & -0.056314\end{array}$

H $\quad 1.262252 \quad-1.256855 \quad-0.952739$

H $\quad 3.897224-0.142026 \quad-0.136463$

H $\quad 3.116779-1.274059 \quad 1.002736$

$\begin{array}{llll}\text { H } & 3.554611 & -1.822937 & -0.653616\end{array}$

serine_26 -569.59097222 a.u.

$\begin{array}{llll}\text { C } & -3.365625 & -0.664387 & 0.016355\end{array}$

C $\quad-2.024299 \quad-0.024972 \quad 0.304893$

$\begin{array}{llll}\text { O } & -1.522386 & 0.013097 & 1.430844\end{array}$

H $\quad-1.708016 \quad 0.160547 \quad-1.684808$

$\begin{array}{llll}N & -1.385237 & 0.532836 & -0.796138\end{array}$

$\begin{array}{llll}\text { C } & 0.062488 & 0.710077 & -0.711620\end{array}$

$\begin{array}{llll}\text { C } & 0.739112 & -0.650642 & -0.414875\end{array}$

O $\quad 0.173044 \quad-1.716945 \quad-0.695004$

$\begin{array}{llll}\text { C } & 0.437816 & 1.841261 & 0.264401\end{array}$

$\begin{array}{llll}\text { O } & 1.810007 & 2.259018 & 0.086308\end{array}$

H $\quad 1.875721 \quad 2.725536 \quad-0.759103$

$\begin{array}{llll}H & 2.395588 & 0.328823 & 0.275979\end{array}$

$\begin{array}{llll}N & 1.982807 & -0.586843 & 0.122096\end{array}$

$\begin{array}{llll}\text { C } & 2.717777 & -1.810415 & 0.418795\end{array}$

H $\quad-3.184390 \quad-1.716010 \quad-0.259233$

$\begin{array}{llll}\text { H } & -3.980037 & -0.636950 & 0.924852\end{array}$

H $\quad-3.892393 \quad-0.166897 \quad-0.811558$

$\begin{array}{llll}\text { H } & 0.397421 & 1.019723 & -1.718753\end{array}$

H $\quad-0.254313 \quad 2.6855920 .112165$

$\begin{array}{llll}H & 0.358847 & 1.498261 & 1.301435\end{array}$

$\begin{array}{llll}\text { H } & 2.143094 & -2.439612 & 1.114249\end{array}$

URL: http://mc.manuscriptcentral.com/tandf/tmph 


$$
\begin{array}{llll}
\text { H } & 2.899485 & -2.389888 & -0.499271 \\
\text { H } & 3.677072 & -1.537960 & 0.877454
\end{array}
$$

serine_27 -569.59115753 a.u.

$\begin{array}{llll}\text { C } & 2.635444 & -1.766647 & -0.064499\end{array}$

$\begin{array}{llll}\text { C } & 1.331236 & -0.996526 & -0.133435\end{array}$

O $\quad 0.588345 \quad-1.026346 \quad-1.118118$

$\begin{array}{llll}H & 2.544084 & -2.676786 & -0.670966\end{array}$

H $\quad 3.427139-1.136085 \quad-0.499751$

$\begin{array}{llll}H & 2.918239 & -2.028300 & 0.966223\end{array}$

$\begin{array}{llll}N & 1.013513 & -0.277358 & 1.009439\end{array}$

C $\quad 0.043823 \quad 0.821603 \quad 0.962536$

$\begin{array}{llll}\text { C } & -1.229279 & 0.403514 & 0.202584\end{array}$

O $\quad-1.729109 \quad 1.134575 \quad-0.660751$

$\begin{array}{llll}\text { C } & 0.635807 & 2.105708 & 0.368111\end{array}$

O $\quad 1.195401 \quad 1.899935-0.923659$

H $\quad 1.760038 \quad-0.179200 \quad 1.691012$

H $\quad 0.467185 \quad 1.589085 \quad-1.486775$

$\begin{array}{llll}H & -0.261965 & 1.032327 & 2.003800\end{array}$

$\begin{array}{llll}H & -0.149793 & 2.881098 & 0.359822\end{array}$

H $\quad 1.465068 \quad 2.449624 \quad 1.007215$

N $\quad-1.803338 \quad-0.759658 \quad 0.630890$

C $\quad-2.843819 \quad-1.367795 \quad-0.196209$

H $\quad-1.181032 \quad-1.384706 \quad 1.133187$

$\begin{array}{llll}H & -3.650641 & -0.640161 & -0.349831\end{array}$

H $\quad-2.443285-1.666259-1.178965$

$\begin{array}{llll}H & -3.242638 & -2.245974 & 0.328417\end{array}$ 
serine_28 -569.59007297 a.u.

$$
\begin{array}{llll}
\text { C } & -3.216704 & -0.696178 & 0.676629 \\
\text { C } & -2.021913 & -0.566542 & -0.244372 \\
\text { O } & -1.701209 & -1.438451 & -1.059664
\end{array}
$$$$
\begin{array}{llll}
H & -3.985394 & -1.306365 & 0.185589
\end{array}
$$$$
\text { H } \quad-2.886447 \quad-1.214759 \quad 1.591114
$$$$
\begin{array}{llll}
H & -3.631230 & 0.283333 & 0.956535
\end{array}
$$$$
\begin{array}{llll}
N & -1.302894 & 0.609579 & -0.120273
\end{array}
$$$$
\begin{array}{llll}
\text { C } & 0.043145 & 0.609502 & -0.658353
\end{array}
$$$$
\begin{array}{llll}
\text { C } & 0.948338 & -0.319405 & 0.175617
\end{array}
$$$$
\begin{array}{llll}
O & 0.682315 & -0.627100 & 1.339808
\end{array}
$$$$
\text { C } \quad 0.585106 \quad 2.036046 \quad-0.684979
$$$$
\begin{array}{llll}
\text { O } & 0.555834 & 2.518630 & 0.666643
\end{array}
$$$$
\begin{array}{llll}
H & -1.392782 & 1.096748 & 0.768550
\end{array}
$$$$
\begin{array}{llll}
H & 0.829259 & 3.445714 & 0.665419
\end{array}
$$$$
\begin{array}{llll}
H & -0.006516 & 0.230311 & -1.692487
\end{array}
$$$$
\begin{array}{llll}
H & 1.614931 & 2.033067 & -1.082784
\end{array}
$$$$
\begin{array}{llll}
H & -0.059816 & 2.649008 & -1.338440
\end{array}
$$$$
\begin{array}{llll}
N & 2.095486 & -0.719118 & -0.458693
\end{array}
$$$$
\begin{array}{llll}
\text { C } & 2.983345 & -1.674846 & 0.199203
\end{array}
$$$$
\text { H } \quad 2.103541 \quad-0.676379-1.471064
$$$$
\begin{array}{llll}
\text { H } & 3.244392 & -1.291690 & 1.194232
\end{array}
$$$$
\begin{array}{llll}
H & 2.502106 & -2.658503 & 0.320844
\end{array}
$$$$
\text { H } \quad 3.896388 \quad-1.779811 \quad-0.401515
$$

serine_29 -569.58914106 a.u.
$\begin{array}{llll}\text { C } & 2.702645 & -2.086065 & 0.129949\end{array}$
$\begin{array}{llll}\text { C } & 2.057523 & -0.714097 & 0.059420\end{array}$

URL: http://mc.manuscriptcentral.com/tandf/tmph 


$$
\begin{array}{llll}
\mathrm{O} & 2.731314 & 0.296810 & -0.188213 \\
\mathrm{H} & 3.347017 & -2.213859 & -0.750655 \\
\mathrm{H} & 3.342525 & -2.120745 & 1.024613 \\
\mathrm{H} & 1.973022 & -2.907310 & 0.176274 \\
\mathrm{~N} & 0.701436 & -0.692555 & 0.270381 \\
\mathrm{C} & -0.112451 & 0.516692 & 0.480220 \\
\mathrm{C} & -1.595314 & 0.135149 & 0.453333 \\
\mathrm{O} & -2.424065 & 0.703371 & 1.171490 \\
\mathrm{C} & 0.101714 & 1.617701 & -0.591567 \\
\mathrm{O} & 1.162618 & 2.507653 & -0.303826 \\
\mathrm{H} & 0.304650 & -1.554458 & 0.632035 \\
\mathrm{H} & 1.959772 & 1.943804 & -0.260035 \\
\mathrm{H} & 0.084667 & 0.966611 & 1.467127 \\
\mathrm{H} & 0.224035 & 1.127565 & -1.578102 \\
\mathrm{H} & -0.812406 & 2.230593 & -0.615872 \\
\mathrm{~N} & -1.938572 & -0.839096 & -0.446965 \\
\mathrm{C} & -3.347612 & -1.140230 & -0.687990 \\
\mathrm{H} & -1.242358 & -1.121641 & -1.127647 \\
\mathrm{H} & -3.839905 & -1.351070 & 0.270601 \\
\mathrm{H} & -3.865516 & -0.291638 & -1.162137 \\
\mathrm{H} & -3.413515 & -2.023863 & -1.335926
\end{array}
$$


$\begin{array}{llll}H & 2.521428 & -1.454016 & -1.961169\end{array}$

N $\quad 0.919918-0.467512 \quad-0.234064$

$\begin{array}{llll}\text { C } & 0.013194 & 0.169596 & 0.716171\end{array}$

$\begin{array}{llll}\text { C } & -1.382747 & -0.447859 & 0.553388\end{array}$

O $\quad-1.859133 \quad-1.218111 \quad 1.396025$

$\begin{array}{llll}\text { C } & 0.049307 & 1.697548 & 0.598937\end{array}$

O $\quad-0.275028 \quad 2.061592 \quad-0.762286$

H $\quad 0.554513 \quad-0.755243 \quad-1.133920$

$\begin{array}{llll}H & -0.143464 & 3.013965 & -0.859671\end{array}$

$\begin{array}{llll}H & 0.350483 & -0.090272 & 1.730658\end{array}$

$\begin{array}{llll}H & -0.679304 & 2.133670 & 1.305476\end{array}$

$\begin{array}{llll}H & 1.063990 & 2.037798 & 0.856805\end{array}$

N $\quad-2.018465 \quad-0.135593 \quad-0.618054$

C $\quad-3.373065 \quad-0.626740 \quad-0.852744$

H $\quad-1.688641 \quad 0.695860 \quad-1.103714$

H $\quad-3.392564 \quad-1.716021 \quad-0.713925$

H $\quad-4.096194 \quad-0.179632-0.151470$

$\begin{array}{llll}\text { H } & -3.661164 & -0.383662 & -1.883933\end{array}$

serine_31 -569.58976019 a.u.

C $\quad-2.263067 \quad-2.254733 \quad-0.056877$

C $\quad-1.149658 \quad-1.258005 \quad 0.200014$

O $\quad-0.504585-1.222996 \quad 1.252641$

H $\quad-2.061388 \quad-3.169131 \quad 0.515490$

$\begin{array}{llll}H & -3.209422 & -1.819550 & 0.302588\end{array}$

H $\quad-2.370911 \quad-2.494153 \quad-1.125325$

N $\quad-0.880662 \quad-0.385583 \quad-0.843230$

C $\quad-0.138070 \quad 0.850099 \quad-0.570972$

URL: http://mc.manuscriptcentral.com/tandf/tmph 


$$
\begin{array}{llll}
\mathrm{C} & 1.302184 & 0.594006 & -0.102971 \\
\mathrm{O} & 1.898086 & 1.429404 & 0.588530 \\
\mathrm{C} & -0.885321 & 1.755107 & 0.417003 \\
\mathrm{O} & -2.204613 & 2.046592 & -0.087426 \\
\mathrm{H} & -1.611775 & -0.306208 & -1.543371 \\
\mathrm{H} & -2.146049 & 2.848210 & -0.623982 \\
\mathrm{H} & -0.058352 & 1.379281 & -1.537285 \\
\mathrm{H} & -1.017581 & 1.228751 & 1.371161 \\
\mathrm{H} & -0.301198 & 2.668626 & 0.596165 \\
\mathrm{~N} & 1.889604 & -0.535642 & -0.590219 \\
\mathrm{C} & 3.193185 & -0.943158 & -0.076611 \\
\mathrm{H} & 1.261713 & -1.244139 & -0.951920 \\
\mathrm{H} & 3.889396 & -0.098032 & -0.153681 \\
\mathrm{H} & 3.130330 & -1.250093 & 0.980188 \\
\mathrm{H} & 3.566027 & -1.778882 & -0.683364
\end{array}
$$$$
\text { serine_32 -569.58956384 a.u. }
$$$$
\text { C } \quad-2.262481 \quad-2.253727 \quad-0.112639
$$$$
\begin{array}{llll}
\text { C } & -1.243826 & -1.174527 & 0.209494
\end{array}
$$$$
\text { O } \quad-0.724776 \quad-1.063125 \quad 1.326255
$$$$
\text { H } \quad-1.471793 \quad-0.370793 \quad-1.667727
$$$$
\text { N } \quad-0.896382 \quad-0.343169 \quad-0.834101
$$$$
\begin{array}{llll}
\text { C } & -0.087707 & 0.859048 & -0.614245
\end{array}
$$$$
\text { C } \quad \begin{array}{llll}
.341247 & 0.551844 & -0.141392
\end{array}
$$$$
\begin{array}{llll}
\text { O } & 1.999023 & 1.409182 & 0.461219
\end{array}
$$$$
\begin{array}{llll}
\text { C } & -0.757341 & 1.857994 & 0.345597
\end{array}
$$$$
\text { O } \quad-2.077971 \quad 2.188122 \quad-0.114630
$$$$
\begin{array}{llll}
H & -2.683390 & 1.518210 & 0.229287
\end{array}
$$ 
H $\quad \begin{array}{llll}1.182911 & -1.360234 & -0.808491\end{array}$

N $\quad 1.848611-0.659515 \quad-0.506853$

$\begin{array}{llll}\text { C } & 3.142979 & -1.084638 & 0.015500\end{array}$

H $\quad-2.602266 \quad-2.232751 \quad-1.158278$

$\begin{array}{llll}H & -1.813661 & -3.234002 & 0.104524\end{array}$

$\begin{array}{llll}H & -3.127596 & -2.128697 & 0.555107\end{array}$

H $\quad 0.000033 \quad 1.347900 \quad-1.599042$

$\begin{array}{llll}\text { H } & -0.771579 & 1.450944 & 1.366716\end{array}$

$\begin{array}{llll}H & -0.174430 & 2.786743 & 0.343097\end{array}$

H $\quad 3.884205 \quad-0.298166 \quad-0.177292$

$\begin{array}{llll}\text { H } & 3.098716 & -1.264119 & 1.101931\end{array}$

H $\quad 3.445822 \quad-2.005642 \quad-0.499790$

serine_33 -569.58936549 a.u.

$\begin{array}{llll}\text { C } & 2.475785 & -2.053480 & -0.104962\end{array}$

C $\quad 1.430630 \quad-0.983725 \quad-0.338442$

O $\quad 0.705849 \quad-0.954067 \quad-1.338906$

$\begin{array}{llll}H & 2.873627 & -2.382786 & -1.073295\end{array}$

$\begin{array}{llll}H & 3.295058 & -1.704336 & 0.540484\end{array}$

$\begin{array}{llll}H & 1.982479 & -2.911012 & 0.380261\end{array}$

$\begin{array}{llll}N & 1.324283 & -0.020564 & 0.651226\end{array}$

$\begin{array}{llll}\text { C } & 0.113913 & 0.808349 & 0.675184\end{array}$

C $\quad-1.128049 \quad-0.100300 \quad 0.578995$

$\begin{array}{llll}O & -1.236876 & -1.073151 & 1.334345\end{array}$

$\begin{array}{llll}\text { C } & 0.228514 & 1.944725 & -0.349905\end{array}$

$\begin{array}{llll}\text { O } & 1.383708 & 2.745174 & -0.085455\end{array}$

H $\quad 1.713268-0.273322 \quad 1.553902$

$\begin{array}{llll}H & 2.137020 & 2.134424 & -0.081052\end{array}$

URL: http://mc.manuscriptcentral.com/tandf/tmph 


$$
\begin{array}{llll}
\mathrm{H} & 0.074073 & 1.271790 & 1.674427 \\
\mathrm{H} & 0.260523 & 1.533240 & -1.373092 \\
\mathrm{H} & -0.633346 & 2.621327 & -0.249539 \\
\mathrm{~N} & -2.104559 & 0.273189 & -0.299644 \\
\mathrm{C} & -3.233496 & -0.630273 & -0.516979 \\
\mathrm{H} & -1.815212 & 0.850072 & -1.080938 \\
\mathrm{H} & -3.705833 & -0.850288 & 0.448871 \\
\mathrm{H} & -3.959323 & -0.133415 & -1.173905 \\
\mathrm{H} & -2.905630 & -1.579494 & -0.970407
\end{array}
$$$$
\text { serine_34 -569.58548889 a.u. }
$$$$
\text { C } \quad 3.287362-1.508146 \quad-0.028550
$$$$
\begin{array}{llll}
\text { C } & 2.364738 & -0.306753 & 0.074195
\end{array}
$$$$
\begin{array}{llll}
O & 2.783505 & 0.840620 & 0.234580
\end{array}
$$$$
\begin{array}{llll}
H & 0.758195 & -1.548332 & 0.196578
\end{array}
$$$$
\text { N } \quad 1.009359-0.591784 \quad-0.039184
$$$$
\begin{array}{llll}
\text { C } & 0.029727 & 0.437762 & 0.318817
\end{array}
$$$$
\begin{array}{llll}
\text { C } & -1.373281 & -0.141806 & 0.437597
\end{array}
$$$$
\text { O } \quad-2.242623 \quad 0.433014 \quad 1.110285
$$$$
\begin{array}{llll}
\text { C } & -0.044785 & 1.541016 & -0.766167
\end{array}
$$$$
\text { O } \quad-0.861680 \quad 2.629650 \quad-0.348963
$$$$
\begin{array}{llll}
H & -1.641757 & 2.265065 & 0.102871
\end{array}
$$$$
\begin{array}{llll}
\text { H } & -0.987019 & -1.503722 & -1.034579
\end{array}
$$$$
\begin{array}{llll}
N & -1.636790 & -1.272309 & -0.290236
\end{array}
$$$$
\begin{array}{llll}
\text { C } & -3.016269 & -1.746895 & -0.398412
\end{array}
$$$$
\text { H } 2.768355 \quad-2.423022 \quad-0.349363
$$$$
\text { H } \quad 4.089391 \quad-1.272055 \quad-0.741462
$$$$
\begin{array}{llll}
H & 3.749593 & -1.677782 & 0.955805
\end{array}
$$ 
$\begin{array}{llll}H & 0.286338 & 0.919481 & 1.276653\end{array}$

$\begin{array}{llll}H & 0.962444 & 1.941013 & -0.935297\end{array}$

H $\quad-0.418053 \quad 1.087624 \quad-1.706102$

H $\quad-3.434930 \quad-1.856102 \quad 0.610141$

H $\quad-3.645462 \quad-1.038336 \quad-0.960056$

$\begin{array}{llll}H & -3.013635 & -2.722515 & -0.901347\end{array}$

serine_35 -569.58463816 a.u.

C $\quad-3.288880 \quad-0.819234 \quad-0.424296$

C $\quad \begin{array}{llll}-1.834561 & -0.580782 & -0.051986\end{array}$

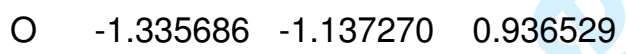

$\begin{array}{llll}H & -3.509933 & -1.888000 & -0.302829\end{array}$

$\begin{array}{llll}\text { H } & -3.918624 & -0.257450 & 0.282748\end{array}$

H $\quad-3.536420 \quad-0.503888-1.448592$

N $\quad-1.164516 \quad 0.271937 \quad-0.893674$

$\begin{array}{llll}\text { C } & 0.142348 & 0.937899 & -0.751922\end{array}$

$\begin{array}{llll}\text { C } & 1.405317 & 0.081023 & -0.532562\end{array}$

$\begin{array}{llll}\text { O } & 2.486328 & 0.545880 & -0.935722\end{array}$

$\begin{array}{llll}\text { C } & 0.117762 & 2.083065 & 0.264366\end{array}$

O $\quad-0.098032 \quad 1.521891 \quad 1.562812$

H $\quad-1.718366 \quad 0.618575 \quad-1.667605$

$\begin{array}{llll}H & -0.005626 & 2.230308 & 2.214423\end{array}$

H $\quad 0.330248 \quad 1.405866 \quad-1.731468$

H $\quad 1.087749 \quad 2.606181 \quad 0.199183$

$\begin{array}{llll}H & -0.694833 & 2.783892 & -0.004221\end{array}$

N $\quad 1.275501 \quad-1.106357 \quad 0.093797$

C $\quad 2.468403 \quad-1.880646 \quad 0.412939$

$\begin{array}{llll}\text { H } & 0.387657 & -1.279798 & 0.578270\end{array}$

URL: http://mc.manuscriptcentral.com/tandf/tmph 


$$
\begin{array}{llll}
H & 3.069236 & -2.027101 & -0.495502 \\
H & 3.094490 & -1.364113 & 1.158551 \\
H & 2.154320 & -2.855487 & 0.807996
\end{array}
$$

serine_36 -569.58518761 a.u.

C $\quad 3.128904 \quad-1.010278-0.318829$

C $\quad 1.710453 \quad-0.470511-0.328149$

O $\quad 0.897950 \quad-0.749991-1.212976$

H $\quad 3.511581 \quad-1.008899 \quad-1.347981$

$\begin{array}{llll}H & 3.803833 & -0.430807 & 0.328313\end{array}$

$\begin{array}{llll}H & 3.098277 & -2.052231 & 0.036123\end{array}$

$\begin{array}{llll}N & 1.388661 & 0.347566 & 0.735073\end{array}$

C $\quad 0.009553 \quad 0.752931 \quad 1.005775$

$\begin{array}{llll}\text { C } & -0.964353 & -0.406390 & 0.692525\end{array}$

O $\quad-0.902710 \quad-1.443946 \quad 1.361609$

C $\quad-0.329581 \quad 2.119451 \quad 0.408988$

O $\quad-0.261770 \quad 2.054098 \quad-1.025550$

$\begin{array}{llll}H & 2.035157 & 0.358927 & 1.514630\end{array}$

H $\quad-0.395503 \quad 2.947728 \quad-1.370621$

$\begin{array}{llll}H & -0.071339 & 0.875571 & 2.099667\end{array}$

H $\quad-1.342684 \quad 2.408299 \quad 0.744201$

$\begin{array}{llll}H & 0.399447 & 2.853530 & 0.794747\end{array}$

N $\quad-1.906393 \quad-0.165856 \quad-0.265592$

C $\quad-2.708460 \quad-1.297052-0.725632$

H $\quad-1.610513 \quad 0.511778 \quad-0.966400$

H $\quad-3.204952 \quad-1.7619690 .135790$

H $\quad-3.467296 \quad-0.928192-1.428505$

H $\quad-2.078738 \quad-2.055896 \quad-1.219069$ 
serine_37 -569.58191740 a.u.

$$
\begin{array}{llll}
\text { C } & -3.448036 & -0.344564 & -0.374990 \\
\text { C } & -2.067579 & -0.117312 & 0.213981
\end{array}
$$

O $\quad-1.856220 \quad 0.649850 \quad 1.155911$

$\begin{array}{llll}H & -3.948946 & 0.627191 & -0.482656\end{array}$

$\begin{array}{llll}H & -3.420352 & -0.856961 & -1.347981\end{array}$

$\begin{array}{llll}H & -4.033544 & -0.950370 & 0.333526\end{array}$

N $\quad-1.034945 \quad-0.815241 \quad-0.386210$

$\begin{array}{llll}\text { C } & 0.278519 & -0.858125 & 0.257787\end{array}$

$\begin{array}{llll}\text { C } & 0.939590 & 0.527594 & 0.331762\end{array}$

$\begin{array}{llll}\mathrm{O} & 1.677284 & 0.837070 & 1.267507\end{array}$

C $\quad \begin{array}{llll}1.149593 & -1.821887 & -0.556702\end{array}$

O $\quad 2.390796 \quad-1.966897 \quad 0.127289$

H $\quad-1.286792 \quad-1.578661 \quad-1.006624$

H $\quad 2.988924 \quad-2.474568 \quad-0.437233$

H $\quad 0.201738 \quad-1.210573 \quad 1.299345$

$\begin{array}{llll}H & 0.624539 & -2.795018 & -0.634847\end{array}$

H $\quad 1.290441 \quad-1.408890 \quad-1.572572$

$\begin{array}{llll}N & 0.726851 & 1.327695 & -0.763051\end{array}$

$\begin{array}{llll}\text { C } & 1.183755 & 2.713781 & -0.717124\end{array}$

H $\quad-0.096990 \quad 1.110952 \quad-1.313221$

H $\quad 2.254866 \quad 2.728948 \quad-0.477342$

H $\quad 1.025182 \quad 3.171529 \quad-1.702782$

$\begin{array}{llll}H & 0.647653 & 3.292137 & 0.053281\end{array}$

serine_38 -569.57795927 a.u.

C $\quad 2.873379 \quad-1.643738 \quad-0.852648$

$\begin{array}{llll}\text { C } & 2.065133 & -0.756007 & 0.077880\end{array}$ 


$$
\begin{aligned}
& \begin{array}{llll}
\mathrm{O} & 2.488694 & -0.392232 & 1.176244
\end{array} \\
& \text { H } \quad 3.237631 \quad-2.508755 \quad-0.281146 \\
& \begin{array}{llll}
H & 2.301669 & -1.993627 & -1.725141
\end{array} \\
& \text { H } \quad 3.747013 \quad-1.072906 \quad-1.201064 \\
& \begin{array}{llll}
N & 0.829548 & -0.379125 & -0.408442
\end{array} \\
& \begin{array}{llll}
\text { C } & -0.060751 & 0.494068 & 0.342427
\end{array} \\
& \begin{array}{llll}
\text { C } & -1.436883 & -0.162293 & 0.505221
\end{array} \\
& \begin{array}{llll}
O & -1.842558 & -0.603319 & 1.584499
\end{array} \\
& \text { C } \quad-0.157711 \quad 1.888221 \quad-0.283546 \\
& \begin{array}{llll}
\text { O } & 1.148016 & 2.463572 & -0.228556
\end{array} \\
& \text { H } \quad 0.520605 \quad-0.759589-1.294602 \\
& \begin{array}{llll}
H & 1.095024 & 3.368113 & -0.566158
\end{array} \\
& \begin{array}{llll}
H & 0.375001 & 0.586315 & 1.347696
\end{array} \\
& \text { H } \quad-0.503670 \quad 1.819779-1.334835 \\
& \begin{array}{llll}
H & -0.891331 & 2.485805 & 0.289024
\end{array} \\
& \begin{array}{llll}
\mathrm{N} & -2.172442 & -0.282018 & -0.649916
\end{array} \\
& \begin{array}{llll}
\text { C } & -3.512169 & -0.862667 & -0.599564
\end{array} \\
& \text { H } \quad-1.909784 \quad 0.268300 \quad-1.457784 \\
& \text { H } \quad-3.460397 \quad-1.851761 \quad-0.125035 \\
& \text { H } \quad-4.201879 \quad-0.235396 \quad-0.013122 \\
& \begin{array}{llll}
H & -3.888815 & -0.967942 & -1.625446
\end{array}
\end{aligned}
$$

\section{CYSTEINE}

cysteine_1 $\quad-892.21081947086$ a.u.

$\begin{array}{llll}\text { C } & -2.250475 & -2.429025 & -0.803597\end{array}$

$\begin{array}{llll}\text { C } & -1.705008 & -1.313294 & 0.063364\end{array}$

O $\quad-2.443769 \quad-0.622773 \quad 0.792264$

$\begin{array}{llll}H & -2.718237 & -3.179529 & -0.149367\end{array}$ 
$\begin{array}{llll}H & -3.032565 & -2.016087 & -1.456741\end{array}$

H $\quad-1.475334 \quad-2.912408 \quad-1.415119$

$\begin{array}{llll}N & -0.358239 & -1.117616 & 0.006890\end{array}$

$\begin{array}{llll}\text { C } & 0.327371 & -0.108090 & 0.807715\end{array}$

C $\quad 0.238126 \quad 1.293364 \quad 0.164326$

$\begin{array}{llll}\text { O } & 1.241303 & 1.917042 & -0.224971\end{array}$

C $\quad 1.782348 \quad-0.525004 \quad 1.040149$

S $\quad 2.681147 \quad-0.977895 \quad-0.500053$

H $\quad 0.208275 \quad-1.609646 \quad-0.680558$

$\begin{array}{llll}H & 2.610078 & 0.269090 & -1.021316\end{array}$

H $\quad-0.196100 \quad-0.063949 \quad 1.779520$

H $\quad 2.322010 \quad 0.287929 \quad 1.544295$

H $\quad 1.809625 \quad-1.424789 \quad 1.673800$

$\begin{array}{llll}N & -1.023164 & 1.778410 & 0.075788\end{array}$

$\begin{array}{llll}\text { C } & -1.278214 & 3.070030 & -0.550455\end{array}$

H $\quad-1.784325 \quad 1.183804 \quad 0.407467$

H $\quad-0.717473 \quad 3.862809 \quad-0.033882$

H $\quad-0.966190 \quad 3.061603 \quad-1.606209$

H $\quad-2.353453 \quad 3.279909-0.487142$

cysteine_2 -892.20733413107 a.u.

$\begin{array}{llll}\text { C } & -3.779963 & -1.000497 & 0.125086\end{array}$

C $\quad-2.552638 \quad-0.167719 \quad-0.188375$

$\begin{array}{llll}O & -2.620707 & 0.938535 & -0.747450\end{array}$

H $\quad-4.452654 \quad-0.407498 \quad 0.761596$

H $\quad-4.306892 \quad-1.213791 \quad-0.816389$

H $\quad-3.535755 \quad-1.945305 \quad 0.631419$

$\begin{array}{llll}N & -1.352565 & -0.699240 & 0.196431\end{array}$

URL: http://mc.manuscriptcentral.com/tandf/tmph 


$$
\begin{array}{llll}
\text { C } & -0.119117 & -0.022078 & -0.146425 \\
\text { C } & 1.009726 & -1.055123 & -0.017833 \\
\text { O } & 0.800504 & -2.187716 & 0.449573 \\
\text { C } & 0.091440 & 1.194582 & 0.785608 \\
\text { S } & 1.272374 & 2.441199 & 0.126578 \\
\text { H } & -1.259249 & -1.652596 & 0.540139 \\
\text { H } & 0.468274 & 2.854183 & -0.879441 \\
\text { H } & -0.187717 & 0.343966 & -1.185900 \\
\text { H } & -0.878258 & 1.684709 & 0.941265 \\
\text { H } & 0.493774 & 0.871713 & 1.758979 \\
\mathrm{~N} & 2.231351 & -0.644850 & -0.440111 \\
\mathrm{C} & 3.397387 & -1.506892 & -0.273791 \\
\mathrm{H} & 2.344417 & 0.336802 & -0.680404 \\
\mathrm{H} & 3.617403 & -1.680981 & 0.791061 \\
\mathrm{H} & 3.210186 & -2.478909 & -0.750785 \\
\mathrm{H} & 4.257597 & -1.023032 & -0.753620
\end{array}
$$

cysteine_3 $\quad-892.20737742594$ a.u.

$\begin{array}{llll}\text { C } & 3.453412 & -1.281508 & 0.523441\end{array}$

$\begin{array}{llll}\text { C } & 2.215467 & -0.757578 & -0.175347\end{array}$

O $\quad 2.279015 \quad-0.126889-1.244419$

H $\quad 3.980631 \quad-1.956834 \quad-0.166067$

H $\quad 4.118577 \quad-0.433174 \quad 0.740149$

H $\quad 3.227096 \quad-1.818487 \quad 1.455760$

$\begin{array}{llll}N & 1.018490 & -1.034948 & 0.428544\end{array}$

C $\quad-0.249306-0.560703 \quad-0.136710$

$\begin{array}{llll}\text { C } & -0.480714 & 0.899623 & 0.294913\end{array}$

O $\quad-1.222700 \quad 1.198054 \quad 1.246003$ 
$\begin{array}{llll}\text { C } & -1.390105 & -1.459657 & 0.328725\end{array}$

S $\quad-2.980843 \quad-1.028295 \quad-0.473449$

$\begin{array}{llll}H & 1.018575 & -1.417538 & 1.368160\end{array}$

$\begin{array}{llll}H & -3.136455 & 0.129468 & 0.206984\end{array}$

H $\quad-0.147099 \quad-0.611156 \quad-1.231629$

$\begin{array}{llll}H & -1.175579 & -2.501094 & 0.042784\end{array}$

H $\quad-1.509875 \quad-1.397143 \quad 1.420737$

$\begin{array}{llll}N & 0.218742 & 1.806260 & -0.432477\end{array}$

C $\quad 0.221985 \quad 3.214254 \quad-0.052476$

$\begin{array}{llll}H & 0.930343 & 1.436487 & -1.062197\end{array}$

$\begin{array}{llll}H & -0.813087 & 3.564270 & 0.060312\end{array}$

$\begin{array}{llll}H & 0.745933 & 3.373398 & 0.903930\end{array}$

H $\quad 0.718843 \quad 3.789427 \quad-0.844161$

cysteine_4 -892.20640260508 a.u.

$\begin{array}{llll}\text { C } & -2.291869 & -2.368868 & -0.833364\end{array}$

C $\quad-1.726852 \quad-1.292512 \quad 0.070467$

$\begin{array}{llll}O & -2.453325 & -0.612504 & 0.819979\end{array}$

$\begin{array}{llll}\text { H } & -2.796282 & -3.119221 & -0.206945\end{array}$

H $\quad-3.047684 \quad-1.914303 \quad-1.489990$

H $\quad-1.521974 \quad-2.862129-1.443667$

$\begin{array}{llll}N & -0.374682 & -1.123872 & 0.024483\end{array}$

$\begin{array}{llll}\text { C } & 0.318058 & -0.118470 & 0.823905\end{array}$

$\begin{array}{llll}\text { C } & 0.250125 & 1.277488 & 0.170647\end{array}$

$\begin{array}{llll}\text { O } & 1.269319 & 1.864880 & -0.225919\end{array}$

C $\quad 1.774584-0.530576 \quad 1.043904$

S $\quad 2.656412 \quad-0.847426 \quad-0.549478$

$\begin{array}{llll}\mathrm{H} & 0.171391 & -1.566894 & -0.710937\end{array}$

URL: http://mc.manuscriptcentral.com/tandf/tmph 


$$
\begin{array}{lrrr}
\text { H } & 2.933473 & -2.147656 & -0.303493 \\
\mathrm{H} & -0.200222 & -0.072622 & 1.797671 \\
\mathrm{H} & 2.302100 & 0.281539 & 1.561164 \\
\mathrm{H} & 1.815272 & -1.443416 & 1.654074 \\
\mathrm{~N} & -1.001657 & 1.790519 & 0.077471 \\
\mathrm{C} & -1.226843 & 3.078253 & -0.567851 \\
\mathrm{H} & -1.775986 & 1.218787 & 0.415187 \\
\mathrm{H} & -0.644374 & 3.864488 & -0.065454 \\
\mathrm{H} & -0.918925 & 3.046218 & -1.624405 \\
\mathrm{H} & -2.296179 & 3.316595 & -0.503977
\end{array}
$$$$
\text { cysteine_5 } \quad-892.20648452460 \text { a.u. }
$$$$
\begin{array}{llll}
\text { C } & 3.365309 & -0.457956 & -0.966367
\end{array}
$$$$
\begin{array}{llll}
\text { C } & 2.306650 & -0.508623 & 0.119937
\end{array}
$$$$
\begin{array}{llll}
\text { O } & 2.545941 & -0.919973 & 1.259138
\end{array}
$$$$
\text { H } \quad 3.871696 \quad-1.432341 \quad-1.011211
$$$$
\text { H } \quad 2.953812 \quad-0.208472 \quad-1.955245
$$$$
\text { H } \quad 4.114303 \quad 0.297748 \quad-0.685406
$$$$
\text { N } \quad \begin{array}{llll}
1.047243 & -0.085495 & -0.267561
\end{array}
$$$$
\begin{array}{llll}
\text { C } & 0.007599 & 0.170911 & 0.718670
\end{array}
$$$$
\begin{array}{llll}
\text { C } & -1.302774 & -0.564928 & 0.402333
\end{array}
$$$$
\begin{array}{llll}
O & -2.383883 & -0.185360 & 0.878618
\end{array}
$$$$
\begin{array}{llll}
\text { C } & -0.263415 & 1.670818 & 0.881924
\end{array}
$$$$
\begin{array}{llll}
\text { S } & -0.786072 & 2.457067 & -0.698267
\end{array}
$$$$
\text { H } \quad 0.965721 \quad 0.437521 \quad-1.137125
$$$$
\begin{array}{llll}
H & -2.000968 & 1.862636 & -0.724410
\end{array}
$$$$
\begin{array}{llll}
\text { H } & 0.385494 & -0.233973 & 1.674398
\end{array}
$$$$
\begin{array}{llll}
H & -1.038589 & 1.835246 & 1.641390
\end{array}
$$ 
$\begin{array}{llll}H & 0.662921 & 2.182914 & 1.184114\end{array}$

N $\quad-1.189550 \quad-1.653609 \quad-0.399459$

$\begin{array}{llll}\text { C } & -2.351036 & -2.485135 & -0.690703\end{array}$

H $\quad-0.267371 \quad-1.897836 \quad-0.739031$

H $\quad-3.147766 \quad-1.876232 \quad-1.142113$

H $\quad-2.050574 \quad-3.274650 \quad-1.391310$

H $\quad-2.745851 \quad-2.939755 \quad 0.230545$

cysteine_6 $\quad-892.20587470359$ a.u.

C $\quad 0.630535 \quad 3.168306 \quad 0.719844$

$\begin{array}{llll}\text { C } & 0.092708 & 1.952937 & -0.006196\end{array}$

O $\quad-0.690372 \quad 2.052903 \quad-0.968863$

H $\quad 1.281345 \quad 0.720300 \quad 1.118803$

$\begin{array}{llll}N & 0.503515 & 0.738546 & 0.466546\end{array}$

$\begin{array}{llll}\text { C } & 0.223084 & -0.492953 & -0.272537\end{array}$

C $\quad-1.174454-1.029581 \quad 0.112049$

O $\quad-1.320801 \quad-2.006065 \quad 0.862881$

$\begin{array}{llll}\text { C } & 1.269952 & -1.564344 & 0.030782\end{array}$

$\begin{array}{llll}S & 3.002304 & -0.996643 & -0.188787\end{array}$

H $\quad 2.871023 \quad-0.603975 \quad-1.476046$

$\begin{array}{llll}\text { H } & -1.962230 & 0.549163 & -0.891267\end{array}$

N $\quad-2.200983 \quad-0.337961 \quad-0.445065$

C $\quad-3.576880-0.638358-0.066306$

H $\quad-0.218887 \quad 3.759068 \quad 1.091712$

$\begin{array}{llll}H & 1.290561 & 2.908107 & 1.559525\end{array}$

H $\quad 1.182970 \quad 3.787182 \quad-0.002332$

H $\quad 0.226305 \quad-0.242218-1.348600$

H $\quad 1.186089 \quad-1.883077 \quad 1.080819$

URL: http://mc.manuscriptcentral.com/tandf/tmph 


$$
\begin{array}{llll}
\text { H } & 1.077457 & -2.445813 & -0.595377 \\
\text { H } & -4.250979 & -0.062699 & -0.713504 \\
\text { H } & -3.768401 & -1.711910 & -0.199617 \\
\text { H } & -3.770144 & -0.382685 & 0.988143
\end{array}
$$

cysteine_7 $\quad-892.20580222661$ a.u.

$\begin{array}{llll}\text { C } & 3.830761 & -0.896459 & -0.226683\end{array}$

$\begin{array}{llll}\text { C } & 2.605398 & -0.140534 & 0.249977\end{array}$

$\begin{array}{llll}O & 2.679696 & 0.851213 & 0.990307\end{array}$

H $\quad 4.483269 \quad-0.197466 \quad-0.769786$

H $\quad 4.381673 \quad-1.257482 \quad 0.653973$

$\begin{array}{llll}H & 3.583109 & -1.747618 & -0.877107\end{array}$

N $\quad 1.400071 \quad-0.625846 \quad-0.180282$

$\begin{array}{llll}\text { C } & 0.159129 & 0.027205 & 0.192588\end{array}$

$\begin{array}{llll}\text { C } & -0.967196 & -0.966732 & -0.086905\end{array}$

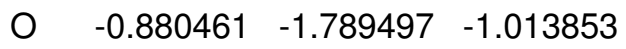

C $\quad-0.024274 \quad 1.314855 \quad-0.639102$

$\begin{array}{llll}\text { S } & -1.471709 & 2.333269 & -0.146445\end{array}$

$\begin{array}{llll}H & 1.326807 & -1.410762 & -0.821606\end{array}$

H $\quad-2.404214 \quad 1.601387 \quad-0.795068$

$\begin{array}{llll}H & 0.204484 & 0.299348 & 1.259575\end{array}$

$\begin{array}{llll}H & 0.855528 & 1.952088 & -0.466487\end{array}$

H $\quad-0.087513 \quad 1.065601 \quad-1.708790$

$\begin{array}{llll}N & -2.049831 & -0.876325 & 0.728544\end{array}$

$\begin{array}{llll}\text { C } & -3.250615 & -1.661097 & 0.458087\end{array}$

H $\quad-2.100372 \quad-0.076461 \quad 1.350141$

$\begin{array}{llll}H & -2.967821 & -2.711910 & 0.311988\end{array}$

H $\quad-3.925281 \quad-1.579063 \quad 1.319750$

URL: http://mc.manuscriptcentral.com/tandf/tmph 
cysteine_8 -892.20482964037 a.u.

C $\quad 0.604532 \quad 3.161764 \quad 0.744382$

C $\quad 0.103053 \quad 1.946643 \quad-0.007465$

$\begin{array}{llll}\text { O } & -0.630617 & 2.044038 & -1.008023\end{array}$

H $\quad-0.256886 \quad 3.788183 \quad 1.015600$

H $\quad 1.1711592 .901798 \quad 1.649942$

$\begin{array}{llll}H & 1.248428 & 3.743788 & 0.068395\end{array}$

$\begin{array}{llll}N & 0.503318 & 0.733003 & 0.473793\end{array}$

$\begin{array}{llll}\text { C } & 0.227606 & -0.510795 & -0.249291\end{array}$

C $\quad-1.177173 \quad-1.039704 \quad 0.111889$

O $\quad-1.343899 \quad-2.030771 \quad 0.839090$

$\begin{array}{llll}\text { C } & 1.273842 & -1.566086 & 0.094121\end{array}$

S $\quad 2.942421 \quad-0.894069 \quad-0.312602$

H $\quad 1.153666 \quad 0.700979 \quad 1.251855$

H $\quad 3.629099-1.994624 \quad 0.062770$

$\begin{array}{llll}\text { H } & 0.256589 & -0.270305 & -1.326244\end{array}$

H $\quad 1.209732-1.825116 \quad 1.160255$

H $\quad 1.074607 \quad-2.467631 \quad-0.499881$

N $\quad-2.188496 \quad-0.320146 \quad-0.436614$

C $\quad-3.573585-0.615598-0.089866$

H $\quad-1.934999 \quad 0.557461 \quad-0.892877$

H $\quad-3.779388 \quad-1.680511 \quad-0.265999$

H $\quad-3.778335 \quad-0.396587 \quad 0.970509$

H $\quad-4.229677 \quad-0.005824-0.724105$

cysteine_9 $\quad-892.20471439220$ a.u. 


$$
\begin{array}{llll}
\mathrm{C} & 0.039098 & 3.214162 & 0.797306 \\
\mathrm{C} & -0.114054 & 1.954424 & -0.030270 \\
\mathrm{O} & -0.681828 & 1.955005 & -1.135035 \\
\mathrm{H} & 0.706373 & 0.818919 & 1.478874 \\
\mathrm{~N} & 0.433370 & 0.815702 & 0.500816 \\
\mathrm{C} & 0.316083 & -0.477656 & -0.176940 \\
\mathrm{C} & -1.046256 & -1.115185 & 0.156028 \\
\mathrm{O} & -1.166572 & -2.032246 & 0.982722 \\
\mathrm{C} & 1.459990 & -1.394285 & 0.244165 \\
\mathrm{~S} & 3.089934 & -0.823637 & -0.378985 \\
\mathrm{H} & 2.986488 & 0.437990 & 0.094291 \\
\mathrm{H} & -1.894842 & 0.278056 & -1.062832 \\
\mathrm{~N} & -2.086424 & -0.567853 & -0.527118 \\
\mathrm{C} & -3.456966 & -0.965747 & -0.226088 \\
\mathrm{H} & -0.958969 & 3.636390 & 0.982417 \\
\mathrm{H} & 0.549065 & 3.040679 & 1.755867 \\
\mathrm{H} & 0.611758 & 3.946538 & 0.209557 \\
\mathrm{H} & 0.373439 & -0.266883 & -1.256405 \\
\mathrm{H} & 1.477481 & -1.518234 & 1.337117 \\
\mathrm{H} & 1.304908 & -2.390646 & -0.194713 \\
\mathrm{H} & -4.124357 & -0.510023 & -0.968679 \\
\hline & -3.542067 & -2.059804 & -0.279259 \\
\hline
\end{array}
$$


$\begin{array}{llll}H & 4.091716 & -0.404494 & 0.701937\end{array}$

H $\quad 3.240413 \quad-1.803785 \quad 1.438335$

$\begin{array}{llll}\text { H } & 3.986190 & -1.940323 & -0.187770\end{array}$

$\begin{array}{llll}N & 1.007076 & -1.051215 & 0.443558\end{array}$

$\begin{array}{llll}\text { C } & -0.261958 & -0.553357 & -0.096667\end{array}$

$\begin{array}{llll}\text { C } & -0.446868 & 0.912663 & 0.336576\end{array}$

$\begin{array}{llll}\text { O } & -1.063848 & 1.216797 & 1.367845\end{array}$

$\begin{array}{llll}\text { C } & -1.421082 & -1.409217 & 0.400509\end{array}$

S $\quad \begin{array}{llll}-3.000101 & -0.933650 & -0.404074\end{array}$

H $\quad 1.033279 \quad-1.358626 \quad 1.410414$

H $\quad-2.750328 \quad-1.560485-1.575379$

H $\quad-0.177132 \quad-0.615151 \quad-1.192477$

$\begin{array}{llll}\text { H } & -1.217303 & -2.476011 & 0.228453\end{array}$

H $\quad-1.578355 \quad-1.231642 \quad 1.475383$

$\begin{array}{llll}N & 0.162751 & 1.811242 & -0.481082\end{array}$

C $\quad 0.221255 \quad 3.220235 \quad-0.108449$

H $\quad 0.821974 \quad 1.432875 \quad-1.158986$

$\begin{array}{llll}H & -0.789666 & 3.569869 & 0.140340\end{array}$

$\begin{array}{llll}H & 0.868657 & 3.381836 & 0.768963\end{array}$

$\begin{array}{llll}\text { H } & 0.607430 & 3.793413 & -0.961138\end{array}$

cysteine_11 -892.20436303871 a.u.

$\begin{array}{llll}\text { C } & 3.511505 & -0.953218 & -0.779221\end{array}$

C $\quad 2.450561 \quad-0.466059 \quad 0.188846$

$\begin{array}{llll}\mathrm{O} & 2.722383 & -0.065165 & 1.329707\end{array}$

H $\quad 4.261090 \quad-0.158955 \quad-0.906999$

H $\quad 4.015372 \quad-1.823878-0.334427$

H $\quad 3.099500 \quad-1.229827 \quad-1.760276$

URL: http://mc.manuscriptcentral.com/tandf/tmph 


$$
\begin{array}{llll}
\text { N } & 1.163233 & -0.475382 & -0.284006 \\
\text { C } & 0.058506 & -0.182157 & 0.607041 \\
\text { C } & -1.197781 & -0.796708 & -0.025741 \\
\text { O } & -1.153211 & -1.405550 & -1.104662 \\
\text { C } & -0.080405 & 1.333440 & 0.840934 \\
\text { S } & -0.613220 & 2.245827 & -0.659492 \\
\text { H } & 0.912858 & -0.986768 & -1.128682 \\
\text { H } & 0.433348 & 1.848674 & -1.417430 \\
\text { H } & 0.253521 & -0.656591 & 1.588073 \\
\text { H } & -0.846358 & 1.545780 & 1.604720 \\
\text { H } & 0.878745 & 1.721599 & 1.210237 \\
\mathrm{~N} & -2.348526 & -0.648439 & 0.681964 \\
\mathrm{C} & -3.621446 & -1.084787 & 0.114718 \\
\mathrm{H} & -2.345732 & -0.064283 & 1.507751 \\
\mathrm{H} & -3.874530 & -0.497339 & -0.781352 \\
\mathrm{H} & -3.554482 & -2.143809 & -0.169235 \\
\mathrm{H} & -4.403773 & -0.958443 & 0.873950
\end{array}
$$

cysteine_12 -892.20449191511 a.u.

$\begin{array}{llll}\text { C } & -1.786700 & 2.689061 & 0.544688\end{array}$

C $\quad-0.917816 \quad 1.550353 \quad 0.048906$

O $\quad-0.522400 \quad 1.500197 \quad-1.132224$

$\begin{array}{llll}H & -1.308085 & 3.639346 & 0.269802\end{array}$

$\begin{array}{llll}H & -2.755349 & 2.636461 & 0.024877\end{array}$

$\begin{array}{llll}H & -1.958524 & 2.662434 & 1.630169\end{array}$

$\begin{array}{llll}N & -0.602772 & 0.593979 & 0.966319\end{array}$

$\begin{array}{llll}\text { C } & 0.065116 & -0.679141 & 0.674272\end{array}$

$\begin{array}{llll}\text { C } & 1.587886 & -0.564253 & 0.469417\end{array}$

URL: http://mc.manuscriptcentral.com/tandf/tmph 
$\begin{array}{llll}\text { O } & 2.360974 & -1.325519 & 1.072234\end{array}$

C $\quad-0.550881 \quad-1.442704 \quad-0.513915$

S $\quad \begin{array}{llll}-2.354369 & -1.725257 & -0.341197\end{array}$

H $\quad-0.997875 \quad 0.693728 \quad 1.893105$

$\begin{array}{llll}\text { H } & -2.724594 & -0.494921 & -0.759897\end{array}$

H $\quad-0.053266 \quad-1.294635 \quad 1.577474$

H $\quad-0.356297 \quad-0.932878-1.465090$

H $\quad-0.086595 \quad-2.440986-0.545286$

N $\quad 1.994908 \quad 0.367761 \quad-0.427496$

C $\quad 3.411454 \quad 0.518251 \quad-0.737135$

H $\quad 1.279365 \quad 0.896628-0.927556$

H $\quad 3.814277 \quad-0.399704 \quad-1.192423$

H $\quad 3.526845 \quad 1.356668 \quad-1.435968$

$\begin{array}{llll}H & 3.982103 & 0.722969 & 0.180705\end{array}$

cysteine_13 -892.20415032168 a.u.

$\begin{array}{llll}\text { C } & 3.362888 & -0.982904 & -0.923683\end{array}$

$\begin{array}{llll}\text { C } & 2.294666 & -0.787978 & 0.133770\end{array}$

$\begin{array}{llll}\mathrm{O} & 2.556463 & -0.695091 & 1.342571\end{array}$

H $\quad 0.814157 \quad-0.634445 \quad-1.324296$

N $\quad 1.008100 \quad-0.716456 \quad-0.329961$

$\begin{array}{llll}\text { C } & -0.064917 & -0.330456 & 0.558068\end{array}$

C $\quad-1.382043 \quad-0.549981-0.182421$

O $\quad-1.451912 \quad-0.504045 \quad-1.420663$

C $\quad 0.037675 \quad 1.139454 \quad 1.032608$

S $\quad 0.162540 \quad 2.361761 \quad-0.327196$

H $\quad-0.945523 \quad 1.963799 \quad-0.993715$

H $\quad-2.345722 \quad-0.866480 \quad 1.599779$

URL: http://mc.manuscriptcentral.com/tandf/tmph 


$$
\begin{array}{llll}
\text { N } & -2.477539 & -0.718451 & 0.607189 \\
\text { C } & -3.800860 & -0.893041 & 0.015044 \\
\text { H } & 4.108721 & -1.695099 & -0.545966 \\
\text { H } & 2.951527 & -1.341523 & -1.878323 \\
\text { H } & 3.863665 & -0.016727 & -1.091344 \\
\text { H } & -0.016946 & -0.965019 & 1.461053 \\
\text { H } & -0.815891 & 1.380846 & 1.685487 \\
\text { H } & 0.967651 & 1.248165 & 1.610252 \\
\text { H } & -4.547358 & -0.890802 & 0.819377 \\
\text { H } & -4.006671 & -0.065640 & -0.677975 \\
\text { H } & -3.863044 & -1.838385 & -0.545368
\end{array}
$$

cysteine_14 -892.20407501523 a.u.

$$
\text { C } \quad \begin{array}{llll}
-1.849657 & 2.673025 & 0.528754
\end{array}
$$$$
\text { C } \quad-0.885127 \quad 1.605062 \quad 0.055740
$$$$
\begin{array}{llll}
O & -0.392217 & 1.628868 & -1.087781
\end{array}
$$$$
\text { H } \quad-1.521403 \quad 3.644854 \quad 0.137582
$$$$
\begin{array}{llll}
H & -2.842236 & 2.447224 & 0.108481
\end{array}
$$$$
\begin{array}{llll}
H & -1.930537 & 2.719037 & 1.624555
\end{array}
$$$$
\begin{array}{llll}
N & -0.601913 & 0.619371 & 0.957577
\end{array}
$$$$
\begin{array}{llll}
\text { C } & 0.040001 & -0.659793 & 0.634162
\end{array}
$$$$
\begin{array}{llll}
\text { C } & 1.569314 & -0.592596 & 0.456808
\end{array}
$$$$
\begin{array}{llll}
\text { O } & 2.297448 & -1.418688 & 1.031221
\end{array}
$$$$
\text { C } \quad-0.583742-1.363065-0.585623
$$$$
\text { S } \quad-2.388341 \quad-1.646400 \quad-0.416314
$$$$
\text { H } \quad-1.115659 \quad 0.648943 \quad 1.829863
$$$$
\text { H } \quad-2.298261 \quad-2.596782 \quad 0.541342
$$$$
\begin{array}{llll}
H & -0.099607 & -1.300415 & 1.517481
\end{array}
$$ 
H $\quad-0.468296 \quad-0.745389-1.486877$

H $\quad-0.067586 \quad-2.321528 \quad-0.744503$

$\begin{array}{llll}N & 2.030750 & 0.363521 & -0.383843\end{array}$

$\begin{array}{llll}\text { C } & 3.458420 & 0.473445 & -0.656054\end{array}$

$\begin{array}{llll}\text { H } & 1.345226 & 0.955089 & -0.856772\end{array}$

H $\quad 3.837395 \quad-0.441699-1.136544$

H $\quad 3.619756 \quad 1.331888 \quad-1.320600$

$\begin{array}{llll}H & 4.015717 & 0.623033 & 0.280635\end{array}$

cysteine_15 -892.20296328273 a.u.

$\begin{array}{llll}\text { C } & 3.192541 & -1.034334 & -0.980308\end{array}$

$\begin{array}{llll}\text { C } & 2.199831 & -0.799667 & 0.143599\end{array}$

$\begin{array}{llll}\text { O } & 2.491306 & -0.972619 & 1.330322\end{array}$

H $\quad 3.597561 \quad-2.051811 \quad-0.884016$

H $\quad 2.749346 \quad-0.909425 \quad-1.979055$

H $\quad 4.027928 \quad-0.328565 \quad-0.860615$

N $\quad 0.932756 \quad-0.411746 \quad-0.259641$

$\begin{array}{llll}\text { C } & -0.024861 & 0.089923 & 0.716751\end{array}$

C $\quad-1.460446 \quad-0.352915 \quad 0.399169$

O $\quad-2.435691 \quad 0.262942 \quad 0.850339$

C $\quad 0.030804 \quad 1.614361 \quad 0.867502$

S $\quad-0.281874 \quad 2.477235 \quad-0.732086$

H $\quad 0.839686 \quad-0.032831 \quad-1.199339$

H $\quad 1.014723 \quad 2.627822 \quad-1.081005$

H $\quad 0.256244 \quad-0.366104 \quad 1.682273$

$\begin{array}{llll}\mathrm{H} & -0.763179 & 1.937444 & 1.554031\end{array}$

H $\quad 1.007613 \quad 1.924508 \quad 1.264247$

N $\quad-1.579203 \quad-1.464811 \quad-0.374674$

URL: http://mc.manuscriptcentral.com/tandf/tmph 


$$
\begin{array}{llll}
\text { C } & -2.892057 & -2.038903 & -0.644701 \\
\text { H } & -0.731072 & -1.947310 & -0.645267 \\
\text { H } & -3.556277 & -1.263259 & -1.050920 \\
\text { H } & -2.778469 & -2.846848 & -1.378967 \\
\text { H } & -3.348779 & -2.436860 & 0.274856
\end{array}
$$

cysteine_16 -892.20224663189 a.u.

C $\quad 2.647402-2.234413 \quad-0.413084$

$\begin{array}{llll}\text { C } & 1.784274 & -1.211729 & 0.297180\end{array}$

$\begin{array}{llll}O & 2.070415 & -0.767330 & 1.420294\end{array}$

H $\quad 0.415775 \quad-1.221016 \quad-1.274249$

$\begin{array}{llll}N & 0.684644 & -0.785833 & -0.397013\end{array}$

$\begin{array}{llll}\text { C } & -0.242731 & 0.172088 & 0.165083\end{array}$

$\begin{array}{llll}\text { C } & -1.658990 & -0.270842 & -0.210302\end{array}$

O $\quad-1.894141 \quad-0.803026-1.305330$

$\begin{array}{llll}\text { C } & -0.038045 & 1.603119 & -0.370722\end{array}$

S $\quad \begin{array}{llll}1.687163 & 2.211342 & -0.185581\end{array}$

H $1.949135 \quad 1.531995 \quad 0.955329$

H $\quad-2.356736 \quad 0.339865 \quad 1.616196$

$\begin{array}{llll}N & -2.631119 & 0.022961 & 0.694850\end{array}$

$\begin{array}{llll}\text { C } & -4.025435 & -0.319221 & 0.427509\end{array}$

H $2.920865 \quad-3.022440 \quad 0.301860$

$\begin{array}{llll}H & 2.150744 & -2.679039 & -1.287704\end{array}$

$\begin{array}{llll}H & 3.571303 & -1.734406 & -0.741468\end{array}$

$\begin{array}{llll}H & -0.092637 & 0.166414 & 1.257788\end{array}$

H $\quad-0.258128 \quad 1.623968 \quad-1.450268$

$\begin{array}{llll}H & -0.736648 & 2.282156 & 0.143127\end{array}$

H $\quad-4.654934 \quad 0.147204 \quad 1.195974$

URL: http://mc.manuscriptcentral.com/tandf/tmph 
H $\quad-4.310219 \quad 0.062321 \quad-0.562430$

H $\quad-4.176828 \quad-1.409550 \quad 0.436574$

cysteine_17 -892.20286745792 a.u.

$\begin{array}{llll}\text { C } & -0.512386 & 3.294322 & -0.377796\end{array}$

$\begin{array}{llll}\text { C } & 0.021526 & 2.004517 & 0.215788\end{array}$

$\begin{array}{llll}\text { O } & 0.863764 & 1.973598 & 1.117621\end{array}$

$\begin{array}{llll}\mathrm{H} & 0.313671 & 4.012097 & -0.470090\end{array}$

H $\quad-0.988650 \quad 3.142145 \quad-1.357313$

H $\quad-1.254308 \quad 3.7168620 .317785$

$\begin{array}{llll}N & -0.485023 & 0.838241 & -0.335903\end{array}$

$\begin{array}{llll}\text { C } & -0.290036 & -0.412864 & 0.388467\end{array}$

$\begin{array}{llll}\text { C } & 1.148036 & -0.947427 & 0.325091\end{array}$

O $\quad 1.521025 \quad-1.807845 \quad 1.131803$

C $\quad-1.193933-1.508285-0.193742$

S $\quad \begin{array}{llll} & -2.976794 & -1.060434 & -0.230077\end{array}$

H $\quad-1.374075 \quad 0.918885 \quad-0.827127$

H $\quad-3.105728 \quad-0.889853 \quad 1.105247$

$\begin{array}{llll}\text { H } & -0.504349 & -0.275514 & 1.463201\end{array}$

H $\quad-0.925575 \quad-1.698798 \quad-1.245924$

$\begin{array}{llll}H & -1.046234 & -2.433486 & 0.378882\end{array}$

N $\quad 1.903435 \quad-0.503547 \quad-0.716930$

$\begin{array}{llll}\text { C } & 3.313999 & -0.866989 & -0.798765\end{array}$

H $\quad 1.571364 \quad 0.327150 \quad-1.193236$

H $\quad 3.410987 \quad-1.952732 \quad-0.668661$

H $\quad 3.696682 \quad-0.581179-1.787383$

H $\quad 3.904484 \quad-0.367173 \quad-0.013957$

URL: http://mc.manuscriptcentral.com/tandf/tmph 
cysteine_18 -892.20247180682 a.u.

$$
\begin{array}{llll}
\mathrm{C} & -1.915259 & 2.588281 & 0.523677 \\
\mathrm{C} & -0.928778 & 1.542406 & 0.047740 \\
\mathrm{O} & -0.447263 & 1.567506 & -1.098996 \\
\mathrm{H} & -1.616718 & 3.565682 & 0.122766 \\
\mathrm{H} & -2.904952 & 2.332243 & 0.113894 \\
\mathrm{H} & -1.987546 & 2.639593 & 1.619943 \\
\mathrm{~N} & -0.619431 & 0.563102 & 0.950585 \\
\mathrm{C} & 0.073417 & -0.693437 & 0.633114 \\
\mathrm{C} & 1.598734 & -0.573253 & 0.450689 \\
\mathrm{O} & 2.356831 & -1.374793 & 1.020331 \\
\mathrm{C} & -0.538606 & -1.415001 & -0.578138 \\
\mathrm{~S} & -2.353633 & -1.576706 & -0.293676 \\
\mathrm{H} & -1.060445 & 0.617825 & 1.860749 \\
\mathrm{H} & -2.556299 & -2.547124 & -1.210376 \\
\mathrm{H} & -0.050717 & -1.336525 & 1.516484 \\
\mathrm{H} & -0.357136 & -0.854166 & -1.503521 \\
\mathrm{H} & -0.083716 & -2.414229 & -0.639822 \\
\mathrm{~N} & 2.024393 & 0.407388 & -0.381048 \\
\mathrm{C} & 3.446356 & 0.566422 & -0.658716 \\
\mathrm{H} & 1.316786 & 0.965331 & -0.862182 \\
\mathrm{H} & 3.850484 & -0.322876 & -1.166906 \\
\mathrm{H} & 3.578372 & 1.447881 & -1.299195 \\
\hline & 4.003568 & 0.706015 & 0.279357
\end{array}
$$




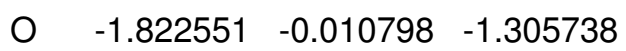

H $\quad-1.739638 \quad-0.791304 \quad 1.768909$

N $\quad-1.340975 \quad-0.607079 \quad 0.856154$

C $\quad 0.129463 \quad-0.579356 \quad 0.761866$

$\begin{array}{llll}\text { C } & 0.668484 & 0.862168 & 0.660991\end{array}$

O $\quad 1.256814 \quad 1.391340 \quad 1.614500$

C $\quad 0.645849 \quad-1.506201 \quad-0.349434$

S $\quad \begin{array}{llll}2.477012 & -1.615591 & -0.340097\end{array}$

H $\quad 2.686659 \quad-0.335371 \quad-0.721133$

H $\quad-0.230188 \quad 1.058213 \quad-1.151173$

$\begin{array}{llll}N & 0.479547 & 1.471449 & -0.543095\end{array}$

$\begin{array}{llll}\text { C } & 0.847094 & 2.875086 & -0.702147\end{array}$

$\begin{array}{llll}H & -4.165617 & 0.509580 & -0.060281\end{array}$

H $\quad-4.120019 \quad-1.214613 \quad-0.491659$

H $\quad-3.870213 \quad-0.720181 \quad 1.214268$

$\begin{array}{llll}\text { H } & 0.506520 & -0.945657 & 1.727735\end{array}$

H $\quad 0.287784 \quad-2.530418 \quad-0.157771$

H $\quad 0.289973 \quad-1.194797 \quad-1.338520$

$\begin{array}{llll}H & 0.706968 & 3.155239 & -1.754210\end{array}$

$\begin{array}{llll}H & 0.235746 & 3.530087 & -0.060057\end{array}$

$\begin{array}{llll}H & 1.900786 & 3.012023 & -0.422899\end{array}$

cysteine_20 -892.20163042237 a.u.

$\begin{array}{llll}\text { C } & 3.479346 & -0.888263 & -0.870725\end{array}$

$\begin{array}{llll}\text { C } & 2.412063 & -0.595657 & 0.165546\end{array}$

$\begin{array}{llll}\text { O } & 2.682368 & -0.354207 & 1.352719\end{array}$

H $\quad 4.155660 \quad-1.656612 \quad-0.470709$

$\begin{array}{llll}\text { H } & 3.060956 & -1.224672 & -1.830207\end{array}$

URL: http://mc.manuscriptcentral.com/tandf/tmph 


$$
\begin{array}{llll}
\mathrm{H} & 4.065095 & 0.028812 & -1.034233 \\
\mathrm{~N} & 1.123925 & -0.605420 & -0.288984 \\
\mathrm{C} & 0.024941 & -0.276074 & 0.589453 \\
\mathrm{C} & -1.266650 & -0.700044 & -0.117565 \\
\mathrm{O} & -1.296904 & -0.929661 & -1.335607 \\
\mathrm{C} & -0.013279 & 1.223978 & 0.947729 \\
\mathrm{~S} & -0.386986 & 2.212774 & -0.563396 \\
\mathrm{H} & 0.890591 & -0.773483 & -1.263767 \\
\mathrm{H} & -0.029836 & 3.400649 & -0.029123 \\
\mathrm{H} & 0.148106 & -0.833841 & 1.535061 \\
\mathrm{H} & -0.786653 & 1.404467 & 1.711058 \\
\mathrm{H} & 0.971106 & 1.501810 & 1.347598 \\
\mathrm{~N} & -2.371880 & -0.767536 & 0.669966 \\
\mathrm{C} & -3.684277 & -1.022402 & 0.082661 \\
\mathrm{H} & -2.290142 & -0.552605 & 1.655309 \\
\mathrm{H} & -3.946051 & -0.230024 & -0.634438 \\
\mathrm{H} & -3.680127 & -1.985184 & -0.447742 \\
\mathrm{H} & -4.427817 & -1.051283 & 0.889174
\end{array}
$$

cysteine_21 -892.20145215785 a.u.

$$
\begin{array}{llll}
\text { C } & -3.693405 & -0.396087 & 0.184280 \\
\text { C } & -2.218737 & -0.300921 & -0.155609 \\
\text { O } & -1.836793 & -0.008109 & -1.302870 \\
\text { H } & -4.146305 & -1.165264 & -0.458242 \\
\text { H } & -3.882438 & -0.645664 & 1.238129 \\
\text { H } & -4.167753 & 0.566369 & -0.055471 \\
\text { N } & -1.353617 & -0.564722 & 0.868933 \\
\text { C } & 0.115631 & -0.567374 & 0.766358
\end{array}
$$




$$
\begin{array}{llll}
\mathrm{C} & 0.708038 & 0.851315 & 0.652950 \\
\mathrm{O} & 1.396978 & 1.321994 & 1.570578 \\
\mathrm{C} & 0.599671 & -1.525517 & -0.333687 \\
\mathrm{~S} & 2.426816 & -1.553283 & -0.464477 \\
\mathrm{H} & -1.752094 & -0.728227 & 1.785546 \\
\mathrm{H} & 2.663247 & -1.951139 & 0.805956 \\
\mathrm{H} & 0.481852 & -0.933104 & 1.737947 \\
\mathrm{H} & 0.213643 & -2.537248 & -0.136177 \\
\mathrm{H} & 0.240178 & -1.203056 & -1.320130 \\
\mathrm{~N} & 0.434276 & 1.508875 & -0.501591 \\
\mathrm{C} & 0.921511 & 2.868386 & -0.698389 \\
\mathrm{H} & -0.273836 & 1.108046 & -1.116607 \\
\mathrm{H} & 2.012372 & 2.893185 & -0.565200 \\
\mathrm{H} & 0.668765 & 3.184122 & -1.718824 \\
\mathrm{H} & 0.470965 & 3.565546 & 0.026215
\end{array}
$$

cysteine_22 -892.20181857175 a.u.

$$
\begin{array}{llll}
\text { C } & 1.102074 & 2.986967 & -0.082923 \\
\text { C } & 0.395113 & 1.677871 & 0.208287 \\
\text { O } & -0.164081 & 1.433290 & 1.282163 \\
\text { H } & 0.666512 & 3.774438 & 0.544863 \\
\text { H } & 2.166680 & 2.876093 & 0.179745 \\
\text { H } & 1.033139 & 3.267708 & -1.144632 \\
\text { N } & 0.399579 & 0.758925 & -0.829690 \\
\text { C } & 0.111965 & -0.648988 & -0.542765 \\
\text { C } & -1.343870 & -0.875941 & -0.093951 \\
\text { O } & -1.649089 & -1.865230 & 0.582963 \\
\text { C } & 1.078943 & -1.266243 & 0.478613
\end{array}
$$




$$
\begin{array}{llll}
\text { S } & 2.832761 & -1.131610 & -0.052328 \\
\text { H } & 1.091604 & 0.923634 & -1.554785 \\
\text { H } & 2.781317 & -2.097734 & -0.996712 \\
\text { H } & 0.219825 & -1.185380 & -1.501871 \\
\text { H } & 1.007476 & -0.723694 & 1.431939 \\
\text { H } & 0.815217 & -2.315622 & 0.657635 \\
\text { N } & -2.256044 & 0.009730 & -0.583171 \\
\mathrm{C} & -3.635841 & -0.045061 & -0.112187 \\
\mathrm{H} & -1.889206 & 0.882283 & -0.944236 \\
\mathrm{H} & -4.013033 & -1.070033 & -0.223074 \\
\mathrm{H} & -3.709471 & 0.241756 & 0.949397 \\
\mathrm{H} & -4.243928 & 0.635610 & -0.722440
\end{array}
$$

cysteine_23 -892.20174893625 a.u.

$$
\begin{array}{llll}
\mathrm{C} & 1.165494 & 2.962890 & -0.106197 \\
\mathrm{C} & 0.485616 & 1.643621 & 0.210875 \\
\mathrm{O} & 0.005282 & 1.387380 & 1.320361 \\
\mathrm{H} & 0.583272 & 3.777753 & 0.345083 \\
\mathrm{H} & 2.163034 & 2.962912 & 0.360529 \\
\mathrm{H} & 1.277298 & 3.138321 & -1.186217 \\
\mathrm{~N} & 0.415747 & 0.739381 & -0.831326 \\
\mathrm{C} & 0.074991 & -0.661819 & -0.575068 \\
\mathrm{C} & -1.384582 & -0.849926 & -0.120908 \\
\mathrm{O} & -1.723655 & -1.869404 & 0.492397 \\
\mathrm{C} & 1.016793 & -1.349234 & 0.425911 \\
\mathrm{~S} & 2.772860 & -1.279169 & -0.103036 \\
\mathrm{H} & 1.004767 & 0.922295 & -1.636892 \\
\mathrm{H} & 2.996190 & -0.001762 & 0.276982
\end{array}
$$


H $\quad 0.166044 \quad-1.177935 \quad-1.545889$

H $\quad 0.908750 \quad-0.913088 \quad 1.426702$

$\begin{array}{llll}H & 0.753936 & -2.414391 & 0.481359\end{array}$

N $\quad-2.263221 \quad 0.109146 \quad-0.524333$

$\begin{array}{llll}\text { C } & -3.640460 & 0.074542 & -0.044303\end{array}$

H $\quad-1.867723 \quad 0.985872 \quad-0.840926$

H $\quad-4.059014 \quad-0.924029 \quad-0.225637$

$\begin{array}{llll}H & -3.694850 & 0.285201 & 1.036007\end{array}$

$\begin{array}{llll}H & -4.225277 & 0.821632 & -0.596829\end{array}$

cysteine_24 -892.20071350906 a.u.

C $\quad-0.700967 \quad 3.252451-0.399680$

C $\quad-0.110184 \quad 2.000100 \quad 0.219215$

O $\quad 0.667381 \quad 2.019533 \quad 1.177572$

$\begin{array}{llll}\text { H } & 0.051507 & 4.051570 & -0.373923\end{array}$

H $\quad-1.042541 \quad 3.089962 \quad-1.432567$

H $\quad-1.560350 \quad 3.570609 \quad 0.211432$

N $\quad-0.489935 \quad 0.806304 \quad-0.372007$

$\begin{array}{llll}\text { C } & -0.253376 & -0.441141 & 0.350650\end{array}$

$\begin{array}{llll}\text { C } & 1.203752 & -0.926259 & 0.294445\end{array}$

O $\quad \begin{array}{llll}1.581467 & -1.829728 & 1.050390\end{array}$

C $\quad-1.119725 \quad-1.560623 \quad-0.236225$

S $\quad \begin{array}{llll}-2.900288 & -1.102148 & -0.067983\end{array}$

H $\quad-1.317757 \quad 0.829108 \quad-0.963887$

H $\quad-3.375026 \quad-2.030378-0.925814$

H $\quad-0.477197 \quad-0.304377 \quad 1.423341$

H $\quad-0.878337 \quad-1.698436 \quad-1.301680$

H $\quad-0.910710 \quad-2.484895 \quad 0.317554$

URL: http://mc.manuscriptcentral.com/tandf/tmph 


$$
\begin{array}{llll}
\text { N } & 1.981211 & -0.372206 & -0.674707 \\
\text { C } & 3.406209 & -0.678810 & -0.729775 \\
\text { H } & 1.629521 & 0.471811 & -1.111277 \\
\text { H } & 3.539649 & -1.767393 & -0.679602 \\
\text { H } & 3.813078 & -0.302940 & -1.677726 \\
\text { H } & 3.948788 & -0.221716 & 0.113408
\end{array}
$$

cysteine_25 -892.19944915731 a.u.

$$
\text { C } \quad 2.644242 \quad-2.266022 \quad-0.408406
$$$$
\text { C } \quad \begin{array}{llll}
1.777603 & -1.241269 & 0.296839
\end{array}
$$$$
\text { O } \quad 2.046150 \quad-0.802208 \quad 1.422550
$$$$
\begin{array}{llll}
H & 2.913081 & -3.053715 & 0.308562
\end{array}
$$$$
\text { H } \quad 2.152185 \quad-2.711070-1.285636
$$$$
\begin{array}{llll}
H & 3.570299 & -1.767072 & -0.732348
\end{array}
$$$$
\text { N } \quad 0.689138 \quad-0.803963 \quad-0.415471
$$$$
\begin{array}{llll}
\text { C } & -0.228931 & 0.174084 & 0.129812
\end{array}
$$$$
\text { C } \quad-1.651409 \quad-0.265089-0.221494
$$$$
\begin{array}{llll}
\text { O } & -1.907919 & -0.790861 & -1.314840
\end{array}
$$$$
\begin{array}{llll}
\text { C } & -0.009772 & 1.575851 & -0.468698
\end{array}
$$$$
\begin{array}{llll}
\text { S } & 1.674041 & 2.172532 & -0.018913
\end{array}
$$$$
\text { H } \quad 0.421180 \quad-1.244093 \quad-1.290236
$$$$
\begin{array}{llll}
H & 1.618499 & 3.279596 & -0.792797
\end{array}
$$$$
\begin{array}{llll}
H & -0.066081 & 0.202003 & 1.220290
\end{array}
$$$$
\text { H } \quad-0.104645 \quad 1.511404 \quad-1.563264
$$$$
\text { H } \quad-0.770152 \quad 2.266642 \quad-0.071884
$$$$
\begin{array}{llll}
N & -2.605009 & 0.020334 & 0.706094
\end{array}
$$$$
\begin{array}{llll}
\text { C } & -4.002746 & -0.328655 & 0.467849
\end{array}
$$$$
\text { H } \quad-2.310195 \quad 0.326133 \quad 1.624988
$$ 
H $\quad-4.313353 \quad 0.060347 \quad-0.511382$

H $\quad-4.146829 \quad-1.420068 \quad 0.469568$

$\begin{array}{llll}H & -4.617323 & 0.125941 & 1.255289\end{array}$

cysteine_26 -892.20064029699 a.u.

C $\quad 2.997166 \quad-1.471871 \quad-1.030993$

C $\quad 2.196993 \quad-0.702582 \quad 0.004229$

$\begin{array}{llll}\text { O } & 2.735085 & 0.003574 & 0.868071\end{array}$

H $\quad 3.649177 \quad-0.765687 \quad-1.565193$

H $\quad 3.640774-2.193271 \quad-0.506496$

H $2.366236 \quad-2.007008 \quad-1.755452$

$\begin{array}{llll}N & 0.834747 & -0.821345 & -0.086871\end{array}$

$\begin{array}{llll}\text { C } & -0.060805 & -0.227290 & 0.900822\end{array}$

C $\quad-1.510056 \quad-0.605592 \quad 0.585785$

O $\quad \begin{array}{llll}-2.292031 & -0.970305 & 1.470991\end{array}$

$\begin{array}{llll}\text { C } & 0.076456 & 1.312631 & 1.005437\end{array}$

S $\quad 0.084613 \quad 2.194992 \quad-0.605102$

H $\quad 0.439818 \quad-1.471153 \quad-0.756823$

$\begin{array}{llll}H & 1.360818 & 1.879629 & -0.919300\end{array}$

$\begin{array}{llll}H & 0.154288 & -0.634948 & 1.903169\end{array}$

H $\quad-0.788182 \quad 1.702673 \quad 1.566151$

H $\quad 0.996777 \quad 1.567002 \quad 1.543636$

$\begin{array}{llll}N & -1.860062 & -0.505105 & -0.737223\end{array}$

C $\quad-3.265306 \quad-0.665128-1.109404$

H $\quad-1.283486 \quad 0.127704 \quad-1.291508$

H $\quad-3.628964 \quad-1.627920 \quad-0.727667$

H $\quad-3.893384 \quad 0.134090 \quad-0.683562$

H $\quad-3.341604 \quad-0.652989-2.204410$

URL: http://mc.manuscriptcentral.com/tandf/tmph 
cysteine_27 -892.20045719276 a.u.

$$
\begin{aligned}
& \begin{array}{llll}
\text { C } & 1.395409 & 2.832856 & -0.071384
\end{array} \\
& \text { C } \quad 0.576057 \quad 1.592403 \quad 0.222169 \\
& \begin{array}{llll}
\text { O } & 0.054880 & 1.365135 & 1.317757
\end{array} \\
& \begin{array}{llll}
H & 1.053463 & 3.648326 & 0.578418
\end{array} \\
& \begin{array}{llll}
H & 2.449427 & 2.613423 & 0.163544
\end{array} \\
& \begin{array}{llll}
H & 1.327900 & 3.137496 & -1.126717
\end{array} \\
& \begin{array}{llll}
N & 0.433694 & 0.708960 & -0.836803
\end{array} \\
& \begin{array}{llll}
\text { C } & 0.029455 & -0.677895 & -0.580035
\end{array} \\
& \text { C } \quad-1.434745 \quad-0.802448-0.122554 \\
& \text { O } \quad \begin{array}{llll}
-1.815489 & -1.804292 & 0.495025
\end{array} \\
& \text { C } \quad 0.939683 \quad-1.397236 \quad 0.425644 \\
& \text { S } \quad 2.681440 \quad-1.166521 \quad-0.135869 \\
& \begin{array}{llll}
H & 1.033629 & 0.865015 & -1.640868
\end{array} \\
& \begin{array}{llll}
H & 3.208517 & -2.177729 & 0.585743
\end{array} \\
& \begin{array}{llll}
\text { H } & 0.099300 & -1.199420 & -1.550026
\end{array} \\
& \begin{array}{llll}
H & 0.805299 & -0.976403 & 1.429700
\end{array} \\
& \begin{array}{llll}
H & 0.685287 & -2.464026 & 0.428771
\end{array} \\
& \begin{array}{llll}
N & -2.268085 & 0.194581 & -0.528880
\end{array} \\
& \begin{array}{llll}
\text { C } & -3.643358 & 0.228504 & -0.043498
\end{array} \\
& \begin{array}{llll}
H & -1.828212 & 1.052683 & -0.839148
\end{array} \\
& \text { H } \quad-4.105307 \quad-0.753763 \quad-0.207407 \\
& \begin{array}{llll}
H & -3.684187 & 0.458815 & 1.033463
\end{array} \\
& \begin{array}{llll}
H & -4.197547 & 0.991279 & -0.606084
\end{array}
\end{aligned}
$$


$\begin{array}{llll}\text { O } & 1.571803 & -0.544687 & 1.300881\end{array}$

$\begin{array}{llll}H & 3.901587 & -1.431520 & 0.357881\end{array}$

$\begin{array}{llll}H & 2.903378 & -2.893029 & 0.524570\end{array}$

H $\quad 3.169196 \quad-2.184678-1.100415$

N $\quad 0.939501 \quad-0.926912 \quad-0.856925$

C $\quad-0.384177 \quad-0.323594 \quad-0.659753$

C $\quad-0.257860 \quad 1.107647 \quad-0.098122$

$\begin{array}{llll}\text { O } & -1.085136 & 1.571848 & 0.695213\end{array}$

$\begin{array}{llll}\text { C } & -1.300808 & -1.183841 & 0.225661\end{array}$

S $\quad-3.082925 \quad-0.844698 \quad-0.046198$

H $\quad 1.063643 \quad-1.537121 \quad-1.657694$

$\begin{array}{llll}H & -3.028530 & 0.407878 & 0.458861\end{array}$

H $\quad-0.841901 \quad-0.237231 \quad-1.659801$

H $\quad-1.163042 \quad-2.242463 \quad-0.046425$

H $\quad-1.047083 \quad-1.048462 \quad 1.284945$

$\begin{array}{llll}N & 0.769014 & 1.843480 & -0.612363\end{array}$

$\begin{array}{llll}\text { C } & 1.063642 & 3.153483 & -0.039594\end{array}$

$\begin{array}{llll}\text { H } & 1.515327 & 1.325028 & -1.060535\end{array}$

$\begin{array}{llll}\text { H } & 0.158933 & 3.774461 & -0.072972\end{array}$

H $\quad 1.389650 \quad 3.066924 \quad 1.009547$

H $\quad 1.853243 \quad 3.628572 \quad-0.636480$

cysteine_29 -892.19926080892 a.u.

$\begin{array}{llll}\text { C } & -3.687937 & -0.466515 & 0.228138\end{array}$

C $\quad-2.223020 \quad-0.334585-0.140763$

$\begin{array}{llll}\text { O } & -1.871304 & -0.023942 & -1.292916\end{array}$

H $\quad-4.188287 \quad 0.487734 \quad 0.009261$

H $\quad-4.136878 \quad-1.238240 \quad-0.414087$

URL: http://mc.manuscriptcentral.com/tandf/tmph 


$$
\begin{array}{llll}
\mathrm{H} & -3.849961 & -0.732066 & 1.282556 \\
\mathrm{~N} & -1.332676 & -0.584404 & 0.865062 \\
\mathrm{C} & 0.133033 & -0.559711 & 0.742845 \\
\mathrm{C} & 0.709168 & 0.866353 & 0.627777 \\
\mathrm{O} & 1.477430 & 1.305373 & 1.497638 \\
\mathrm{C} & 0.622574 & -1.490337 & -0.377789 \\
\mathrm{~S} & 2.465221 & -1.529892 & -0.303614 \\
\mathrm{H} & -1.710280 & -0.769675 & 1.786259 \\
\mathrm{H} & 2.613610 & -2.337789 & -1.375952 \\
\mathrm{H} & 0.521468 & -0.931271 & 1.703455 \\
\mathrm{H} & 0.220442 & -2.499258 & -0.201310 \\
\mathrm{H} & 0.296299 & -1.124262 & -1.358319 \\
\mathrm{~N} & 0.343063 & 1.561180 & -0.476933 \\
\mathrm{C} & 0.836513 & 2.916813 & -0.684105 \\
\mathrm{H} & -0.369671 & 1.155138 & -1.082988 \\
\mathrm{H} & 0.475738 & 3.596180 & 0.104248 \\
\mathrm{H} & 1.935918 & 2.921961 & -0.664324 \\
\mathrm{H} & 0.484360 & 3.268830 & -1.662271
\end{array}
$$

cysteine_30 -892.19936668052 a.u.

$$
\begin{array}{llll}
\text { C } & 3.659957 & -0.258078 & -0.009699 \\
\text { C } & 2.188227 & -0.442957 & 0.291014 \\
\text { O } & 1.710921 & -0.334725 & 1.423458 \\
\text { H } & 4.243191 & -0.492837 & 0.889445 \\
\text { H } & 3.992831 & -0.884238 & -0.850830 \\
\text { H } & 3.818656 & 0.800947 & -0.270460 \\
\text { N } & 1.393876 & -0.755620 & -0.805614 \\
\text { C } & -0.037762 & -0.475640 & -0.694136
\end{array}
$$


C $\quad-0.233149 \quad 1.032294 \quad-0.384812$

O $\quad 0.664071 \quad 1.846854 \quad-0.641227$

$\begin{array}{llll}\text { C } & -0.698692 & -1.463363 & 0.289351\end{array}$

S $\quad-2.515023 \quad-1.666499 \quad 0.064104$

H $\quad 1.803122 \quad-0.486195 \quad-1.696078$

H $\quad-2.474693 \quad-2.022626 \quad-1.239782$

H $\quad-0.463215 \quad-0.651294-1.698386$

$\begin{array}{llll}\text { H } & -0.214396 & -2.443401 & 0.178140\end{array}$

$\begin{array}{llll}H & -0.565497 & -1.126520 & 1.325842\end{array}$

N $\quad-1.433295 \quad 1.397580 \quad 0.133242$

C $\quad-1.722747 \quad 2.805662 \quad 0.381026$

$\begin{array}{llll}H & -2.168386 & 0.699437 & 0.200886\end{array}$

H $\quad-1.709703 \quad 3.383826 \quad-0.556153$

H $\quad-2.714083 \quad 2.879773 \quad 0.846695$

$\begin{array}{llll}H & -0.966461 & 3.228840 & 1.057303\end{array}$

cysteine_31 -892.19910741984 a.u.
$\begin{array}{llll}\text { C } & 3.216666 & -1.248422 & -1.017932\end{array}$
$\begin{array}{llll}\text { C } & 2.359884 & -0.524992 & 0.004034\end{array}$
$\begin{array}{llll}\text { O } & 2.845101 & 0.145817 & 0.923009\end{array}$
H $\quad 3.816796-0.502161 \quad-1.558968$
$\begin{array}{llll}\text { H } & 3.908019 & -1.915961 & -0.483864\end{array}$
H $\quad 2.626823 \quad-1.830259-1.741011$
$\begin{array}{llll}N & 0.999114 & -0.637116 & -0.171850\end{array}$
C $\quad 0.084487 \quad-0.108422 \quad 0.836319$
C $\quad-1.328161 \quad-0.658767 \quad 0.649883$
O $\quad-2.068003 \quad-0.868038 \quad 1.617205$
C $\quad 0.061248 \quad 1.434819 \quad 0.837093$

URL: http://mc.manuscriptcentral.com/tandf/tmph 


$$
\begin{array}{llll}
\mathrm{S} & -0.471741 & 2.194602 & -0.752174 \\
\mathrm{H} & 0.655646 & -1.361136 & -0.793002 \\
\mathrm{H} & -1.798192 & 2.185412 & -0.492070 \\
\mathrm{H} & 0.415970 & -0.421152 & 1.841539 \\
\mathrm{H} & -0.590132 & 1.790434 & 1.648842 \\
\mathrm{H} & 1.083216 & 1.794758 & 1.018045 \\
\mathrm{~N} & -1.705349 & -0.893239 & -0.651962 \\
\mathrm{C} & -3.102497 & -1.235562 & -0.919576 \\
\mathrm{H} & -1.225721 & -0.316084 & -1.341628 \\
\mathrm{H} & -3.377923 & -2.105661 & -0.310203 \\
\mathrm{H} & -3.783891 & -0.407309 & -0.663483 \\
\mathrm{H} & -3.205648 & -1.486206 & -1.983348
\end{array}
$$

cysteine_32 -892.19858623738 a.u.

$$
\begin{array}{lrrr}
\mathrm{C} & 3.489315 & -0.677007 & -0.065923 \\
\mathrm{C} & 2.047336 & -0.217198 & -0.154329 \\
\mathrm{O} & 1.533332 & 0.088361 & -1.246687 \\
\mathrm{H} & 1.852271 & -0.508948 & 1.843052 \\
\mathrm{~N} & 1.362175 & -0.175007 & 1.022329 \\
\mathrm{C} & -0.031390 & 0.247487 & 1.234112 \\
\mathrm{C} & -1.119418 & -0.703936 & 0.709375 \\
\mathrm{O} & -2.183851 & -0.797406 & 1.336439 \\
\mathrm{C} & -0.321822 & 1.710213 & 0.800895 \\
\mathrm{~S} & -1.086806 & 1.991050 & -0.843192 \\
\mathrm{H} & -0.037374 & 1.532066 & -1.560153 \\
\mathrm{H} & -0.086605 & -1.010630 & -1.005236 \\
\mathrm{~N} & -0.841667 & -1.387123 & -0.432686 \\
\mathrm{C} & -1.918893 & -2.123618 & -1.088027
\end{array}
$$


H $\quad 4.124737 \quad 0.090772 \quad-0.530138$

$\begin{array}{llll}\text { H } & 3.828471 & -0.858313 & 0.963888\end{array}$

H $\quad 3.594290 \quad-1.601281 \quad-0.652841$

$\begin{array}{llll}H & -0.159935 & 0.218430 & 2.327311\end{array}$

$\begin{array}{llll}H & -1.066053 & 2.118941 & 1.501310\end{array}$

$\begin{array}{llll}H & 0.600260 & 2.303687 & 0.894445\end{array}$

H $\quad-1.493860 \quad-2.696120 \quad-1.922811$

H $\quad-2.378181 \quad-2.812936-0.367337$

H $\quad-2.699282 \quad-1.440835 \quad-1.462551$

cysteine_33 -892.19861635228 a.u.

C $\quad-3.470899 \quad-1.367935 \quad-0.309820$

$\begin{array}{llll}\text { C } & -2.216876 & -0.733676 & 0.262301\end{array}$

$\begin{array}{llll}O & -2.204691 & -0.095698 & 1.318115\end{array}$

H $\quad-4.306094 \quad-0.663535 \quad-0.196814$

H $\quad-3.362637 \quad-1.650029 \quad-1.367554$

$\begin{array}{llll}\text { H } & -3.704777 & -2.268638 & 0.278576\end{array}$

$\begin{array}{llll}N & -1.063224 & -0.898307 & -0.484277\end{array}$

$\begin{array}{llll}\text { C } & 0.230841 & -0.566759 & 0.117524\end{array}$

$\begin{array}{llll}\text { C } & 0.454163 & 0.948220 & 0.227674\end{array}$

$\begin{array}{llll}\text { O } & 1.310871 & 1.406831 & 0.998970\end{array}$

C $\quad \begin{array}{llll}1.344560 & -1.185076 & -0.746214\end{array}$

$\begin{array}{llll}\text { S } & 2.980324 & -1.237262 & 0.083438\end{array}$

H $\quad-1.078484 \quad-1.582403 \quad-1.234112$

$\begin{array}{llll}H & 2.880421 & 0.008938 & 0.607567\end{array}$

$\begin{array}{llll}\text { H } & 0.280688 & -0.962180 & 1.145085\end{array}$

$\begin{array}{llll}\text { H } & 1.096439 & -2.240209 & -0.950414\end{array}$

H $\quad 1.416997 \quad-0.648775 \quad-1.706319$

URL: http://mc.manuscriptcentral.com/tandf/tmph 


$$
\begin{array}{llll}
\mathrm{N} & -0.258387 & 1.721854 & -0.633909 \\
\mathrm{C} & -0.175725 & 3.175846 & -0.544286 \\
\mathrm{H} & -1.055832 & 1.288692 & -1.084112 \\
\mathrm{H} & 0.878847 & 3.480123 & -0.572540 \\
\mathrm{H} & -0.705599 & 3.612106 & -1.401037 \\
\mathrm{H} & -0.619701 & 3.544499 & 0.394208
\end{array}
$$

cysteine_34 -892.19849231317 a.u.

C $\quad 3.659020 \quad-0.286207 \quad-0.014330$

$\begin{array}{llll}\text { C } & 2.185758 & -0.446939 & 0.292448\end{array}$

O $\quad \begin{array}{llll}1.712045 & -0.316390 & 1.424710\end{array}$

$\begin{array}{llll}H & 4.241792 & -0.526845 & 0.883599\end{array}$

H $3.978952-0.920360 \quad-0.854396$

$\begin{array}{llll}\text { H } & 3.834157 & 0.769180 & -0.279229\end{array}$

$\begin{array}{llll}N & 1.383605 & -0.763547 & -0.795252\end{array}$

$\begin{array}{llll}\text { C } & -0.044207 & -0.476832 & -0.674032\end{array}$

C $\quad \begin{array}{llll}-0.236091 & 1.035769 & -0.391618\end{array}$

O $\quad 0.640122 \quad 1.851085 \quad-0.710172$

$\begin{array}{llll}\text { C } & -0.708634 & -1.424009 & 0.345757\end{array}$

S $\quad-2.481380-1.673223 \quad-0.125866$

H $\quad 1.786582-0.511453 \quad-1.693506$

$\begin{array}{llll}H & -2.876606 & -2.217282 & 1.045461\end{array}$

H $\quad-0.479569-0.671832-1.671206$

H $\quad-0.191173 \quad-2.391773 \quad 0.310998$

H $\quad-0.646844 \quad-1.008563 \quad 1.357737$

N $\quad-1.413723 \quad 1.402383 \quad 0.175009$

C $\quad-1.703509 \quad 2.812716 \quad 0.408705$

$\begin{array}{llll}H & -2.131721 & 0.698361 & 0.321071\end{array}$ 
$\begin{array}{llll}H & -0.934628 & 3.251711 & 1.060693\end{array}$

H $\quad-1.714645 \quad 3.374146 \quad-0.538285$

$\begin{array}{llll}H & -2.684749 & 2.889882 & 0.894743\end{array}$

cysteine_35 -892.19856656126 a.u.

C $\quad 3.313770 \quad-0.639440 \quad-0.482950$

C $\quad \begin{array}{llll}1.872911 & -0.190529 & -0.374524\end{array}$

O $\quad \begin{array}{llll} & 1.116007 & -0.144500 & -1.355380\end{array}$

H $\quad 2.076928 \quad-0.096543 \quad 1.641855$

$\begin{array}{llll}N & 1.448739 & 0.155408 & 0.886964\end{array}$

$\begin{array}{llll}\text { C } & 0.020943 & 0.193241 & 1.227822\end{array}$

C $\quad-0.703024 \quad-1.001582 \quad 0.561878$

O $\quad-0.245605 \quad-2.141051 \quad 0.715611$

$\begin{array}{llll}\text { C } & -0.597348 & 1.595442 & 1.116612\end{array}$

S $\quad-0.847063 \quad 2.293054 \quad-0.578894$

H $\quad 0.038743 \quad 1.458636 \quad-1.185841$

$\begin{array}{llll}\text { H } & -1.989794 & 0.217202 & -0.422882\end{array}$

$\begin{array}{llll}N & -1.874625 & -0.734224 & -0.074339\end{array}$

C $\quad-2.543287 \quad-1.817259-0.791525$

$\begin{array}{llll}\text { H } & 3.711517 & -0.331657 & -1.458255\end{array}$

$\begin{array}{llll}H & 3.942914 & -0.235181 & 0.323721\end{array}$

$\begin{array}{llll}H & 3.329355 & -1.739778 & -0.429291\end{array}$

$\begin{array}{llll}\text { H } & -0.024962 & -0.053191 & 2.304050\end{array}$

H $\quad-1.590673 \quad 1.580229 \quad 1.593890$

$\begin{array}{llll}H & 0.044410 & 2.286676 & 1.683290\end{array}$

H $\quad-3.500208 \quad-1.441707 \quad-1.176760$

H $\quad-1.923442 \quad-2.185961 \quad-1.624739$

H $\quad-2.727585 \quad-2.650701 \quad-0.100836$

URL: http://mc.manuscriptcentral.com/tandf/tmph 


$$
\begin{aligned}
& \text { cysteine_36 -892.19825551109 a.u. } \\
& \begin{array}{llll}
\text { C } & -1.638194 & 2.710046 & -0.050439
\end{array} \\
& \text { C } \quad-0.674815 \quad 1.541096 \quad-0.100495 \\
& \text { O } \quad-0.033348 \quad 1.243683 \quad-1.111340 \\
& \begin{array}{llll}
H & -1.312283 & 3.471962 & -0.769756
\end{array} \\
& \text { H } \quad-2.631725 \quad 2.342376 \quad-0.353854 \\
& \begin{array}{llll}
H & -1.715867 & 3.150452 & 0.955158
\end{array} \\
& \begin{array}{llll}
N & -0.544040 & 0.843211 & 1.092119
\end{array} \\
& \begin{array}{llll}
\text { C } & -0.010776 & -0.519126 & 1.136910
\end{array} \\
& \begin{array}{llll}
\text { C } & 1.242107 & -0.668985 & 0.250108
\end{array} \\
& \begin{array}{llll}
\text { O } & 1.383663 & -1.632901 & -0.508593
\end{array} \\
& \begin{array}{llll}
\text { C } & -1.051442 & -1.608315 & 0.834442
\end{array} \\
& \text { S } \quad-2.028502 \quad-1.351892 \quad-0.692907 \\
& \text { H } \quad-1.219394 \quad 1.075735 \quad 1.814195 \\
& \begin{array}{llll}
H & -0.957566 & -1.322127 & -1.517481
\end{array} \\
& \begin{array}{llll}
H & 0.341146 & -0.685805 & 2.172565
\end{array} \\
& \text { H } \quad-0.541381 \quad-2.581569 \quad 0.795022 \\
& \begin{array}{llll}
H & -1.796143 & -1.633211 & 1.647204
\end{array} \\
& \begin{array}{llll}
N & 2.217996 & 0.262047 & 0.481182
\end{array} \\
& \begin{array}{llll}
\text { C } & 3.314880 & 0.363514 & -0.479826
\end{array} \\
& \begin{array}{llll}
H & 1.889344 & 1.127996 & 0.896719
\end{array} \\
& \begin{array}{llll}
\text { H } & 3.798070 & -0.616930 & -0.575279
\end{array} \\
& \begin{array}{llll}
H & 2.945741 & 0.676186 & -1.470589
\end{array} \\
& \text { H } \quad 4.045320 \quad 1.092776-0.105240
\end{aligned}
$$


$\begin{array}{llll}\text { O } & 1.152220 & -1.303815 & -1.012003\end{array}$

H $\quad 1.670468 \quad-0.152412 \quad 1.887361$

$\begin{array}{llll}N & 1.072605 & -0.314177 & 1.084513\end{array}$

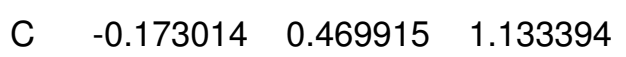

$\begin{array}{llll}\text { C } & -1.495245 & -0.122648 & 0.604438\end{array}$

O $\quad-2.546810 \quad 0.350299 \quad 1.071594$

C $\quad-0.001101 \quad 1.901446 \quad 0.583490$

S $\quad 0.598784 \quad 1.998709 \quad-1.145304$

H $\quad-0.368292 \quad 1.217913 \quad-1.677610$

H $\quad-0.549292 \quad-1.313798 \quad-0.757845$

N $\quad-1.460491 \quad-1.080561 \quad-0.343883$

C $\quad-2.709796-1.612557 \quad-0.875239$

H $\quad 3.338119-2.343535 \quad-0.093252$

$\begin{array}{llll}\text { H } & 3.773882 & -0.618037 & -0.188324\end{array}$

H $\quad 3.395552 \quad-1.347117 \quad 1.402383$

$\begin{array}{llll}H & -0.389848 & 0.582849 & 2.209024\end{array}$

$\begin{array}{llll}H & -0.958668 & 2.431277 & 0.698031\end{array}$

H $\quad 0.765395 \quad 2.431467 \quad 1.172499$

H $\quad-2.469591 \quad-2.422584 \quad-1.575802$

H $\quad-3.332524 \quad-2.003099 \quad-0.057214$

H $\quad-3.284001 \quad-0.830132-1.396004$

cysteine_38 -892.19580040690 a.u.

C $\quad 3.699460 \quad-1.054154 \quad-0.412018$

$\begin{array}{llll}\text { C } & 2.584355 & -0.121854 & 0.021817\end{array}$

$\begin{array}{llll}\text { O } & 2.797750 & 0.982882 & 0.535043\end{array}$

H $\quad 1.155034 \quad-1.518215 \quad-0.541246$

$\begin{array}{llll}N & 1.304815 & -0.579738 & -0.188601\end{array}$

URL: http://mc.manuscriptcentral.com/tandf/tmph 


$$
\begin{array}{llll}
\text { C } & 0.158904 & 0.186678 & 0.303670 \\
\text { C } & -1.015003 & -0.772722 & 0.524535 \\
\text { O } & -1.383677 & -1.110222 & 1.652587 \\
\text { C } & -0.153936 & 1.353322 & -0.644845 \\
\text { S } & -1.668702 & 2.267055 & -0.154048 \\
\text { H } & -1.214966 & 2.637571 & 1.064195 \\
\text { H } & -1.412496 & -0.788062 & -1.481767 \\
\mathrm{~N} & -1.557312 & -1.303768 & -0.621329 \\
\mathrm{C} & -2.764773 & -2.122979 & -0.519349 \\
\mathrm{H} & 4.398897 & -0.492797 & -1.047229 \\
\mathrm{H} & 4.244706 & -1.382760 & 0.485291 \\
\mathrm{H} & 3.334507 & -1.937026 & -0.956797 \\
\mathrm{H} & 0.418109 & 0.594441 & 1.293208 \\
\mathrm{H} & 0.711709 & 2.029189 & -0.678644 \\
\mathrm{H} & -0.339795 & 0.992276 & -1.670317 \\
\mathrm{H} & -2.993954 & -2.536755 & -1.510070 \\
\mathrm{H} & -2.581087 & -2.943308 & 0.186331 \\
\mathrm{H} & -3.620573 & -1.533908 & -0.152562
\end{array}
$$

cysteine_39 -892.19583377205 a.u.

$$
\begin{array}{llll}
\mathrm{C} & 3.083231 & -1.872603 & -0.026662 \\
\mathrm{C} & 1.833021 & -1.047972 & 0.217587 \\
\mathrm{O} & 1.597757 & -0.490333 & 1.293623 \\
\mathrm{H} & 3.951528 & -1.311952 & 0.346486 \\
\mathrm{H} & 3.006093 & -2.802525 & 0.557079 \\
\mathrm{H} & 3.233731 & -2.124470 & -1.086667 \\
\mathrm{~N} & 0.968833 & -0.930592 & -0.854262 \\
\mathrm{C} & -0.366768 & -0.352017 & -0.673240
\end{array}
$$




$$
\begin{array}{llll}
\text { C } & -0.282486 & 1.077211 & -0.100630 \\
\text { O } & -1.103021 & 1.484298 & 0.726245 \\
\text { C } & -1.277461 & -1.212300 & 0.215015 \\
\text { S } & -3.046633 & -0.731801 & 0.032153 \\
\text { H } & 1.116952 & -1.540362 & -1.650973 \\
\text { H } & -3.421414 & -1.836686 & -0.650785 \\
\text { H } & -0.815647 & -0.274471 & -1.677662 \\
\text { H } & -1.170852 & -2.270185 & -0.064892 \\
\text { H } & -0.996211 & -1.082940 & 1.268032 \\
\mathrm{~N} & 0.695154 & 1.864719 & -0.641232 \\
\mathrm{C} & 0.961909 & 3.169236 & -0.041642 \\
\mathrm{H} & 1.455746 & 1.378932 & -1.102503 \\
\mathrm{H} & 0.032468 & 3.753010 & -0.023064 \\
\mathrm{H} & 1.331513 & 3.067832 & 0.991809 \\
\mathrm{H} & 1.707743 & 3.692680 & -0.654359
\end{array}
$$

cysteine_40 -892.19558730201 a.u.

C $\quad 3.651888-1.157321 \quad-0.141174$

$\begin{array}{llll}\text { C } & 2.536053 & -0.149817 & 0.066103\end{array}$

$\begin{array}{llll}O & 2.750704 & 1.039777 & 0.315944\end{array}$

H $\quad 1.140579-1.655584 \quad-0.001178$

N $\quad 1.252293 \quad-0.648013 \quad-0.058928$

$\begin{array}{llll}\text { C } & 0.111924 & 0.183734 & 0.345219\end{array}$

$\begin{array}{llll}\text { C } & -1.131198 & -0.690557 & 0.510732\end{array}$

$\begin{array}{llll}O & -1.911446 & -0.563097 & 1.461064\end{array}$

C $\quad-0.150904 \quad 1.269836 \quad-0.717046$

S $\quad-1.420065 \quad 2.487573 \quad-0.200937$

$\begin{array}{llll}H & -2.336712 & 1.585029 & 0.217626\end{array}$

URL: http://mc.manuscriptcentral.com/tandf/tmph 


$$
\begin{array}{llll}
\mathrm{H} & -0.790062 & -1.515223 & -1.337313 \\
\mathrm{~N} & -1.328264 & -1.616169 & -0.483533 \\
\mathrm{C} & -2.570878 & -2.385086 & -0.520610 \\
\mathrm{H} & 4.356376 & -0.751384 & -0.880629 \\
\mathrm{H} & 4.192159 & -1.277996 & 0.809724 \\
\mathrm{H} & 3.288822 & -2.138349 & -0.480411 \\
\mathrm{H} & 0.310744 & 0.670320 & 1.312959 \\
\mathrm{H} & 0.772891 & 1.849406 & -0.854501 \\
\mathrm{H} & -0.427883 & 0.804429 & -1.676647 \\
\mathrm{H} & -2.483820 & -3.158095 & -1.295119 \\
\mathrm{H} & -2.726469 & -2.861141 & 0.456278 \\
\mathrm{H} & -3.439154 & -1.741493 & -0.733990
\end{array}
$$

cysteine_41 -892.19597944300 a.u.

$$
\begin{array}{llll}
\mathrm{C} & 3.095863 & -1.155546 & -0.553282 \\
\mathrm{C} & 1.729342 & -0.532301 & -0.364109 \\
\mathrm{O} & 0.932130 & -0.359383 & -1.292781 \\
\mathrm{H} & 3.502099 & -0.831279 & -1.520106 \\
\mathrm{H} & 3.795358 & -0.891409 & 0.253382 \\
\mathrm{H} & 2.972926 & -2.249933 & -0.576956 \\
\mathrm{~N} & 1.407828 & -0.166552 & 0.925568 \\
\mathrm{C} & 0.017090 & 0.144988 & 1.257321 \\
\mathrm{C} & -0.929709 & -0.873275 & 0.578854 \\
\mathrm{O} & -0.765144 & -2.079348 & 0.795781 \\
\mathrm{C} & -0.315369 & 1.637775 & 1.120156 \\
\mathrm{~S} & -0.124236 & 2.305389 & -0.596576 \\
\mathrm{H} & 1.982752 & -0.552206 & 1.665910 \\
\mathrm{H} & -0.594666 & 3.530918 & -0.266097
\end{array}
$$


$\begin{array}{llll}H & -0.094799 & -0.078925 & 2.332631\end{array}$

H $\quad-1.353778 \quad 1.796074 \quad 1.450834$

H $\quad 0.362659 \quad 2.196962 \quad 1.781733$

N $\quad-1.969696 \quad-0.367026 \quad-0.144268$

C $\quad-2.791701 \quad-1.303213 \quad-0.908968$

H $\quad-1.805923 \quad 0.548811 \quad-0.559485$

H $\quad-3.194190 \quad-2.066824 \quad-0.230862$

$\begin{array}{llll}\text { H } & -3.620238 & -0.747517 & -1.367450\end{array}$

H $\quad-2.200323 \quad-1.806559-1.691250$

cysteine_42 -892.19409950680 a.u.

C $\quad-3.568131 \quad-1.153410 \quad-0.356039$

$\begin{array}{llll}\text { C } & -2.286367 & -0.601206 & 0.239651\end{array}$

$\begin{array}{llll}\text { O } & -2.261412 & 0.096488 & 1.256473\end{array}$

H $\quad-4.328377 \quad-0.360416 \quad-0.352918$

H $\quad-3.435352 \quad-1.535908 \quad-1.378813$

H $\quad-3.927980 \quad-1.969983 \quad 0.288602$

N $\quad-1.122106 \quad-0.912458-0.439725$

$\begin{array}{llll}\text { C } & 0.169900 & -0.637610 & 0.191039\end{array}$

$\begin{array}{llll}\text { C } & 0.478497 & 0.863378 & 0.282986\end{array}$

O $\quad 1.250326 \quad 1.287142 \quad 1.149442$

$\begin{array}{llll}\text { C } & 1.252812 & -1.348987 & -0.632395\end{array}$

S $\quad 2.919951 \quad-1.067585 \quad 0.101392$

H $\quad-1.165162 \quad-1.636266 \quad-1.150182$

$\begin{array}{llll}H & 3.504864 & -2.059445 & -0.608000\end{array}$

H $\quad 0.174522 \quad-1.003910 \quad 1.230938$

$\begin{array}{llll}H & 1.046491 & -2.430927 & -0.626680\end{array}$

H $\quad 1.231373 \quad-0.971892 \quad-1.667659$

URL: http://mc.manuscriptcentral.com/tandf/tmph 


$$
\begin{array}{llll}
\mathrm{N} & -0.056343 & 1.640040 & -0.703260 \\
\mathrm{C} & 0.103494 & 3.089340 & -0.642122 \\
\mathrm{H} & -0.841128 & 1.247569 & -1.210396 \\
\mathrm{H} & 1.166783 & 3.324425 & -0.503405 \\
\mathrm{H} & -0.246715 & 3.524167 & -1.587603 \\
\mathrm{H} & -0.461941 & 3.522798 & 0.198689
\end{array}
$$

cysteine_43 -892.19324683884 a.u.

$\begin{array}{llll}\text { C } & 3.702306 & -1.063987 & -0.308642\end{array}$

$\begin{array}{llll}\text { C } & 2.583930 & -0.099248 & 0.038732\end{array}$

$\begin{array}{llll}\text { O } & 2.793892 & 1.050417 & 0.440262\end{array}$

H $\quad 4.394433 \quad-0.563646 \quad-1.000394$

H $\quad 4.255450 \quad-1.299595 \quad 0.612777$

H $\quad 3.339848 \quad-1.997791 \quad-0.762615$

N $\quad \begin{array}{lll}1.305071 & -0.582581 & -0.128481\end{array}$

$\begin{array}{llll}\text { C } & 0.158290 & 0.198513 & 0.340504\end{array}$

C $\quad-1.040741 \quad-0.735521 \quad 0.535075$

$\begin{array}{llll}O & -1.599364 & -0.874049 & 1.626728\end{array}$

C $\quad-0.148266 \quad 1.335749 \quad-0.648492$

S $\quad \begin{array}{llll}-1.676638 & 2.200993 & -0.088816\end{array}$

H $\quad 1.171850 \quad-1.570904 \quad-0.312999$

H $\quad-1.582105 \quad 3.218381 \quad-0.972014$

H $\quad 0.387924 \quad 0.632636 \quad 1.325983$

$\begin{array}{llll}H & 0.701886 & 2.030639 & -0.650518\end{array}$

H $\quad-0.292785 \quad 0.928310 \quad-1.662036$

N $\quad-1.413865 \quad-1.438354-0.585449$

C $\quad-2.649850 \quad-2.220061 \quad-0.554608$

H $\quad-1.105183 \quad-1.075244 \quad-1.480866$

URL: http://mc.manuscriptcentral.com/tandf/tmph 
$\begin{array}{llll}\text { H } & -2.609221 & -2.919020 & 0.290685\end{array}$

H $\quad-3.531909 \quad-1.572152 \quad-0.428298$

H $\quad-2.732667 \quad-2.784575 \quad-1.492483$

cysteine_44 -892.19294326763 a.u.

$\begin{array}{llll}\text { C } & 2.259781 & -2.393616 & -0.826023\end{array}$

$\begin{array}{llll}\text { C } & 1.686580 & -1.310332 & 0.066121\end{array}$

$\begin{array}{llll}\text { O } & 2.261939 & -0.923770 & 1.090861\end{array}$

H $\quad 0.041402 \quad-1.157854 \quad-1.173813$

N $\quad 0.496031 \quad-0.767271 \quad-0.356988$

$\begin{array}{llll}\text { C } & -0.194029 & 0.256556 & 0.417544\end{array}$

C $\quad-1.682595 \quad-0.108350 \quad 0.514079$

O $\quad-2.199093 \quad-0.507298 \quad 1.561623$

$\begin{array}{llll}\text { C } & 0.020639 & 1.681638 & -0.118447\end{array}$

S $\quad 1.796855 \quad 2.139905-0.263720$

$\begin{array}{llll}H & 2.217851 & 1.367342 & 0.764884\end{array}$

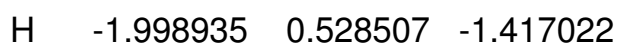

N $\quad-2.382586 \quad-0.032340 \quad-0.666159$

C $\quad-3.816708 \quad-0.316405 \quad-0.673592$

H $\quad 2.556542 \quad-3.244026 \quad-0.196640$

$\begin{array}{llll}H & 1.555739 & -2.733890 & -1.599510\end{array}$

$\begin{array}{llll}\text { H } & 3.163587 & -1.996223 & -1.311875\end{array}$

$\begin{array}{llll}H & 0.202336 & 0.194337 & 1.441901\end{array}$

H $\quad-0.386238 \quad 1.794739 \quad-1.137512$

$\begin{array}{llll}H & -0.502493 & 2.390377 & 0.543589\end{array}$

H $\quad-4.169455 \quad-0.317450-1.713168$

H $\quad-3.989119 \quad-1.304535 \quad-0.227078$

$\begin{array}{llll}\text { H } & -4.379786 & 0.429076 & -0.090162\end{array}$

URL: http://mc.manuscriptcentral.com/tandf/tmph 
cysteine_45 -892.19297152350 a.u.

$$
\begin{array}{llll}
\mathrm{C} & -1.680035 & 2.688722 & 0.011972 \\
\mathrm{C} & -0.742441 & 1.502958 & -0.088041 \\
\mathrm{O} & -0.198027 & 1.160399 & -1.140763 \\
\mathrm{H} & -1.394487 & 3.433233 & -0.742035 \\
\mathrm{H} & -2.698601 & 2.334525 & -0.214667 \\
\mathrm{H} & -1.678175 & 3.146136 & 1.012896 \\
\mathrm{~N} & -0.513801 & 0.828724 & 1.102639 \\
\mathrm{C} & 0.035725 & -0.528134 & 1.109742 \\
\mathrm{C} & 1.312042 & -0.653338 & 0.251509 \\
\mathrm{O} & 1.555067 & -1.682077 & -0.383851 \\
\mathrm{C} & -0.988464 & -1.629044 & 0.791760 \\
\mathrm{~S} & -1.957836 & -1.295422 & -0.742595 \\
\mathrm{H} & -1.122888 & 1.070416 & 1.878199 \\
\mathrm{H} & -2.944452 & -2.164320 & -0.427954 \\
\mathrm{H} & 0.377554 & -0.715098 & 2.144497 \\
\mathrm{H} & -0.441610 & -2.576652 & 0.685773 \\
\mathrm{H} & -1.697921 & -1.697179 & 1.630727 \\
\mathrm{~N} & 2.200277 & 0.379019 & 0.389475 \\
\mathrm{C} & 3.330399 & 0.447891 & -0.534221 \\
\mathrm{H} & 1.796266 & 1.257580 & 0.695446 \\
\mathrm{H} & 3.881020 & -0.500517 & -0.496242 \\
\mathrm{H} & 2.990768 & 0.618125 & -1.569245 \\
\hline
\end{array}
$$


$\begin{array}{llll}O & 2.611505 & -0.353099 & 0.147900\end{array}$

H $\quad-0.096989 \quad-1.969637 \quad 0.265300$

$\begin{array}{llll}N & 0.472239 & -1.150794 & 0.074678\end{array}$

$\begin{array}{llll}\text { C } & -0.111405 & 0.162157 & 0.375459\end{array}$

$\begin{array}{llll}\text { C } & -1.630720 & 0.031223 & 0.479385\end{array}$

$\begin{array}{llll}\text { O } & -2.285978 & 0.633460 & 1.334282\end{array}$

C $\quad 0.229326 \quad 1.205620 \quad-0.734138$

S $\quad \begin{array}{llll}1.216676 & 2.634609 & -0.158540\end{array}$

$\begin{array}{llll}H & 2.297986 & 1.885159 & 0.155563\end{array}$

H $\quad-1.647939-1.050635 \quad-1.263420$

N $\quad-2.212818 \quad-0.779199 \quad-0.465619$

$\begin{array}{llll}\text { C } & -3.669093 & -0.812591 & -0.586794\end{array}$

H $\quad 3.044757 \quad-2.812046 \quad-0.856066$

$\begin{array}{llll}H & 2.872781 & -2.997017 & 0.903669\end{array}$

$\begin{array}{llll}H & 1.520686 & -3.473519 & -0.176007\end{array}$

$\begin{array}{llll}H & 0.249890 & 0.534136 & 1.346490\end{array}$

$\begin{array}{llll}\text { H } & 0.752624 & 0.701748 & -1.559672\end{array}$

H $\quad-0.696483 \quad 1.649656 \quad-1.134243$

H $\quad-3.945218 \quad-1.597551 \quad-1.302844$

H $\quad-4.104993 \quad-1.042885 \quad 0.394090$

H $\quad-4.070685 \quad 0.156656 \quad-0.923240$

cysteine_47 -892.18977633428 a.u.

$\begin{array}{llll}\text { C } & 2.256111 & -2.433423 & -0.804541\end{array}$

$\begin{array}{llll}\text { C } & 1.690433 & -1.328668 & 0.067554\end{array}$

$\begin{array}{llll}\text { O } & 2.274201 & -0.904520 & 1.068383\end{array}$

H $\quad 2.586519 \quad-3.256410 \quad-0.155902$

H $\quad 1.535442 \quad-2.811925 \quad-1.544469$

URL: http://mc.manuscriptcentral.com/tandf/tmph 


$$
\begin{array}{llll}
\text { H } & 3.137703 & -2.038165 & -1.331355 \\
\text { N } & 0.487439 & -0.803470 & -0.355372 \\
\text { C } & -0.187234 & 0.248941 & 0.395944 \\
\text { C } & -1.676047 & -0.102542 & 0.515551 \\
\text { O } & -2.184492 & -0.484571 & 1.572976 \\
\text { C } & 0.029574 & 1.641684 & -0.217416 \\
\text { S } & 1.818892 & 2.073842 & -0.128694 \\
\text { H } & 0.007650 & -1.233647 & -1.137221 \\
\text { H } & 1.691490 & 3.233962 & -0.811874 \\
\text { H } & 0.223717 & 0.226020 & 1.416363 \\
\mathrm{H} & -0.278995 & 1.651029 & -1.275737 \\
\mathrm{H} & -0.562738 & 2.380873 & 0.345166 \\
\mathrm{~N} & -2.388886 & -0.037140 & -0.658292 \\
\mathrm{C} & -3.825468 & -0.307376 & -0.645347 \\
\mathrm{H} & -2.007071 & 0.507787 & -1.421823 \\
\mathrm{H} & -4.002274 & -1.287846 & -0.183851 \\
\mathrm{H} & -4.374954 & 0.450821 & -0.065237 \\
\mathrm{H} & -4.190527 & -0.318674 & -1.680648 \\
\hline
\end{array}
$$

\section{ALANINE PEPTIDES}

Tetrapeptide (extended)
C $\quad-1.7307169 \quad-1.9998193 \quad 2.4398450$
C $\quad-1.7097793 \quad-0.8547348 \quad 1.4381148$
$\begin{array}{llll}\text { O } & -2.7331081 & -0.2460801 & 1.1122687\end{array}$
H $\quad 0.3399725 \quad-1.1321307 \quad 1.1057528$
$\begin{array}{llll}N & -0.4815890 & -0.5546139 & 0.9193524\end{array}$
C $\quad-0.3049099 \quad 0.4209427 \quad-0.1429185$
C $\quad 0.9752746 \quad 0.0212053 \quad-0.9041798$

URL: http://mc.manuscriptcentral.com/tandf/tmph 
$\begin{array}{llll}O & 1.7890205 & -0.7821991 & -0.4277562\end{array}$

C $\quad-0.2141688 \quad 1.8598615 \quad 0.4020435$

$\begin{array}{llll}H & -2.3492830 & -1.7082871 & 3.2978703\end{array}$

$\begin{array}{llll}H & -2.2065653 & -2.8728977 & 1.9702484\end{array}$

$\begin{array}{llll}H & -0.7316886 & -2.2903146 & 2.7925998\end{array}$

$\begin{array}{llll}H & -1.1764994 & 0.3562927 & -0.8156046\end{array}$

H $\quad-0.1251338 \quad 2.5918091 \quad-0.4138146$

$\begin{array}{llll}\text { H } & -1.1259607 & 2.0849493 & 0.9697891\end{array}$

H $\quad 0.6558929 \quad 1.9666558 \quad 1.0649776$

$\begin{array}{llll}H & 0.5042038 & 1.3318417 & -2.4600477\end{array}$

$\begin{array}{llll}N & 1.1499928 & 0.6211133 & -2.1075591\end{array}$

$\begin{array}{llll}\text { C } & 2.3618694 & 0.4597004 & -2.8985189\end{array}$

C $\quad 2.4705975 \quad 1.7126959-3.7914620$

$\begin{array}{llll}\text { O } & 1.4961277 & 2.4520774 & -3.9871938\end{array}$

$\begin{array}{llll}\text { C } & 2.3387540 & -0.8315821 & -3.7387636\end{array}$

$\begin{array}{llll}H & 3.2183819 & 0.4169620 & -2.2060526\end{array}$

H $\quad 3.2732523 \quad-0.9541708 \quad-4.3048091$

H $\quad 2.2232795 \quad-1.6947458 \quad-3.0713953$

H $\quad 1.4994302 \quad-0.8145745 \quad-4.4482407$

$\begin{array}{llll}H & 4.4664927 & 1.2745949 & -4.2320421\end{array}$

N $\quad 3.6863544 \quad 1.9265814 \quad-4.3491845$

$\begin{array}{llll}\text { C } & 3.9380344 & 2.9938027 & -5.3061713\end{array}$

$\begin{array}{llll}\text { C } & 5.1903624 & 2.5711036 & -6.1053237\end{array}$

$\begin{array}{llll}\text { O } & 5.9213548 & 1.6565450 & -5.7116738\end{array}$

C $\quad 4.1392074 \quad 4.3548152 \quad-4.6123572$

H $4.7616485 \quad 4.0125554 \quad-7.4966754$

$\begin{array}{llll}\text { N } & 5.4266357 & 3.2904298 & -7.2357533\end{array}$

$\begin{array}{llll}\text { C } & 6.5940226 & 3.0779876 & -8.0828869\end{array}$

URL: http://mc.manuscriptcentral.com/tandf/tmph 

$\begin{array}{llll}H & 3.0676617 & 3.0633957 & -5.9800374\end{array}$
H $4.2826566 \quad 5.1607312 \quad-5.3464066$
H $\quad 3.2498130 \quad 4.5912478 \quad-4.0150548$
H $\quad 5.0178897 \quad 4.3245858 \quad-3.9534336$
$\begin{array}{llll}H & 7.1639666 & 2.2445437 & -7.6570785\end{array}$
$\begin{array}{llll}H & 6.2929684 & 2.8199577 & -9.1082906\end{array}$
$\begin{array}{llll}H & 7.2323661 & 3.9735146 & -8.1091971\end{array}$

Tetrapeptide (alpha-helix)
$\begin{array}{llll}\text { C } & -5.2517434 & -2.0228398 & 0.7780798\end{array}$
$\begin{array}{llll}\text { C } & -4.8625137 & -0.5517560 & 0.7325422\end{array}$
$\begin{array}{llll}O & -5.5925336 & 0.3304254 & 1.1814779\end{array}$
H $\quad-3.2468464 \quad-1.0469982-0.4047796$
$\begin{array}{llll}\text { N } & -3.6131928 & -0.3039855 & 0.1936698\end{array}$
$\begin{array}{llll}\text { C } & -3.1178851 & 1.0615508 & -0.0685251\end{array}$
$\begin{array}{llll}\text { C } & -1.7262370 & 0.9878734 & -0.7290987\end{array}$
$\begin{array}{llll}\mathrm{O} & -1.4639211 & 1.5783618 & -1.7734027\end{array}$
$\begin{array}{llll}\text { C } & -3.0555640 & 1.9271467 & 1.2022938\end{array}$
$\begin{array}{llll}H & -6.2949817 & -2.1176460 & 0.4506971\end{array}$
H $\quad-4.6105689-2.6679419 \quad 0.1636226$
H $\quad-5.2045696 \quad-2.3636619 \quad 1.8226802$
H $\quad-3.7643212 \quad 1.5600933 \quad-0.8077555$
$\begin{array}{llll}H & -2.6139898 & 2.9036408 & 0.9575397\end{array}$
H $\quad-4.0642812 \quad 2.0735442 \quad 1.6015975$
$\begin{array}{llll}H & -2.4399311 & 1.4514300 & 1.9803264\end{array}$
$\begin{array}{llll}\text { H } & -1.0743581 & -0.1370730 & 0.8696851\end{array}$
$\begin{array}{llll}N & -0.8053020 & 0.2230847 & -0.0443091\end{array}$
C $\quad 0.5751754 \quad 0.0423786-0.4887049$

URL: http://mc.manuscriptcentral.com/tandf/tmph 
$\begin{array}{llll}\text { C } & 0.8320892 & -1.3386175 & -1.1474921\end{array}$

O $1.9299767 \quad-1.6255600 \quad-1.6274862$

$\begin{array}{llll}\text { C } & 1.5748296 & 0.2853630 & 0.6460407\end{array}$

$\begin{array}{llll}H & 0.7306093 & 0.7793531 & -1.2916747\end{array}$

$\begin{array}{llll}H & 2.5927162 & 0.1043997 & 0.2795442\end{array}$

H $\quad 1.5024151 \quad 1.3187428 \quad 1.0101867$

H $\quad 1.3903442 \quad-0.3988468 \quad 1.4890283$

H $\quad-1.1402482-1.8183899 \quad-0.8301867$

N $\quad-0.2371053 \quad-2.1786707 \quad-1.1385930$

C $\quad-0.3020475 \quad-3.4272486-1.8714773$

$\begin{array}{llll}\text { C } & -1.7869380 & -3.6163954 & -2.2563637\end{array}$

$\begin{array}{llll}\mathrm{O} & -2.6893041 & -2.9937801 & -1.6767274\end{array}$

C $\quad 0.2240130 \quad-4.6168323 \quad-1.0456462$

$\begin{array}{llll}\text { H } & -1.2182257 & -4.9588337 & -3.6846560\end{array}$

$\begin{array}{llll}N & -2.0199070 & -4.5168943 & -3.2440336\end{array}$

C $\quad-3.3540002 \quad-4.8607962-3.7221645$

H $\quad 0.3135974 \quad-3.3248260 \quad-2.7813050$

H $\quad 0.2042205 \quad-5.5504236 \quad-1.6266284$

H $\quad 1.2623667 \quad-4.4172411 \quad-0.7507604$

H $\quad-0.3826724-4.7565815 \quad-0.1400023$

H $\quad-4.0694367 \quad-4.2025039 \quad-3.2174844$

H $\quad-3.4263386 \quad-4.7057978 \quad-4.8077443$

$\begin{array}{llll}H & -3.6022480 & -5.9065505 & -3.4893921\end{array}$

Hexapeptide (extended)

C $\quad-0.3608532 \quad-1.6602936 \quad 0.6496324$

C $\quad-0.1276226 \quad-0.5560681 \quad-0.3627130$

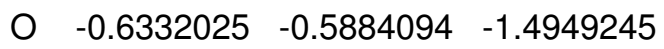

URL: http://mc.manuscriptcentral.com/tandf/tmph 

H $\quad 1.1136119 \quad 0.4378538 \quad 0.9547199$
$\begin{array}{llll}N & 0.6655882 & 0.4785102 & 0.0434593\end{array}$
$\begin{array}{llll}\text { C } & 0.9183939 & 1.6653896 & -0.7855861\end{array}$
$\begin{array}{llll}\text { C } & 2.0432127 & 1.3826641 & -1.8183614\end{array}$
$\begin{array}{llll}\text { O } & 3.1668942 & 1.9025595 & -1.7305239\end{array}$
C $\quad 1.2518372 \quad 2.8647625 \quad 0.0934572$
H $\quad-1.4405553 \quad-1.7411778 \quad 0.8344402$
$\begin{array}{llll}H & -0.0308438 & -2.6144382 & 0.2175455\end{array}$
$\begin{array}{llll}H & 0.1539205 & -1.5005216 & 1.6061688\end{array}$
H $\quad-0.0114654 \quad 1.8462696 \quad-1.3484788$
$\begin{array}{llll}H & 1.3957978 & 3.7592300 & -0.5247347\end{array}$
$\begin{array}{llll}H & 0.4352338 & 3.0558902 & 0.8028889\end{array}$
$\begin{array}{llll}H & 2.1887511 & 2.7007938 & 0.6438141\end{array}$
H $\quad 0.7477894 \quad 0.1077843 \quad-2.7435510$
$\begin{array}{llll}N & 1.6836146 & 0.5323857 & -2.8110937\end{array}$
C $\quad 2.6441820 \quad-0.0159074 \quad-3.7813257$
$\begin{array}{llll}\text { C } & 2.9052129 & 0.9682584 & -4.9560747\end{array}$
$\begin{array}{llll}O & 2.6590893 & 0.6709650 & -6.1352698\end{array}$
C $\quad 2.1639305 \quad-1.3705320 \quad-4.2885396$
H $\quad 3.6043398 \quad-0.1265423 \quad-3.2480599$
H $\quad 2.8886842 \quad-1.7857816 \quad-4.9990594$
$\begin{array}{llll}H & 2.0459071 & -2.0703850 & -3.4503032\end{array}$
H $\quad 1.2059472 \quad-1.2761816 \quad-4.8172679$
H $\quad 3.6489079 \quad 2.2922453 \quad-3.5813462$
N $\quad 3.4488557 \quad 2.1518179 \quad-4.5813551$
C $\quad 3.7113546 \quad 3.2378847 \quad-5.5347050$
$\begin{array}{llll}\text { C } & 5.0764346 & 3.0156435 & -6.2388999\end{array}$
$\begin{array}{llll}O & 6.0670616 & 3.7212666 & -6.0045546\end{array}$

URL: http://mc.manuscriptcentral.com/tandf/tmph 
$\begin{array}{llll}\text { C } & 3.6596470 & 4.5903524 & -4.8345397\end{array}$

H $\quad 2.9260912 \quad 3.1728707 \quad-6.3057079$

$\begin{array}{llll}H & 3.8304095 & 5.3960568 & -5.5592767\end{array}$

H $\quad 2.6775127 \quad 4.7377128 \quad-4.3651184$

H $\quad 4.4454649 \quad 4.6677181 \quad-4.0713944$

H $\quad 4.2282191 \quad 1.4191696 \quad-7.1967284$

$\begin{array}{llll}\text { N } & 5.0832175 & 1.9840880 & -7.1221608\end{array}$

$\begin{array}{llll}\text { C } & 6.3178694 & 1.5017346 & -7.7554772\end{array}$

$\begin{array}{llll}\text { C } & 6.6420725 & 2.3252622 & -9.0346569\end{array}$

O $\quad 6.5579031 \quad 1.8469197-10.1691426$

$\begin{array}{llll}\text { C } & 6.2064926 & 0.0150805 & -8.0722042\end{array}$

$\begin{array}{llll}\text { H } & 7.1311247 & 1.6834701 & -7.0336599\end{array}$

H $\quad 7.1394001 \quad-0.3453565 \quad-8.5223731$

H $\quad 6.0109312 \quad-0.5569795 \quad-7.1547789$

H $\quad 5.4025361 \quad-0.1718295 \quad-8.7963133$

H $\quad 7.0371163 \quad 3.9398624 \quad-7.8015823$

$\begin{array}{llll}N & 7.0364581 & 3.6029448 & -8.7736644\end{array}$

C $\quad 7.3528233 \quad 4.5441033 \quad-9.8391315$

C $\quad 8.5474872 \quad 5.4037628 \quad-9.3875122$

$\begin{array}{llll}\text { O } & 8.6185333 & 5.8621288 & -8.2470668\end{array}$

C $\quad 6.1558599 \quad 5.4501898-10.1861235$

H $\quad 9.3690370 \quad 5.1932384-11.2592796$

N $\quad 9.4824237 \quad 5.6454142 \quad-10.3574375$

C $\quad 10.6466073 \quad 6.4905728-10.1345023$

H $\quad 7.6123680 \quad 3.9350113 \quad-10.7187164$

H $\quad 6.40758926 .1408801 \quad-11.0044347$

H $\quad 5.3047415 \quad 4.8317078-10.5016124$

$\begin{array}{llll}\text { H } & 5.8641729 & 6.0424284 & -9.3081541\end{array}$

URL: http://mc.manuscriptcentral.com/tandf/tmph 


$$
\begin{array}{lrrr}
H & 11.5798958 & 5.9257748 & -10.2774155 \\
H & 10.6456082 & 7.3588508 & -10.8106350 \\
H & 10.5980034 & 6.8434755 & -9.0977057
\end{array}
$$

Hexapeptide (alpha-helix)

C $\quad-1.8566174 \quad 0.1013342 \quad-1.9406248$

C $\quad-0.6159223 \quad 0.8384601 \quad-1.4770731$

$\begin{array}{llll}\text { O } & -0.0408217 & 1.6717919 & -2.1748291\end{array}$

$\begin{array}{llll}H & -0.7847461 & -0.0470457 & 0.3731835\end{array}$

$\begin{array}{llll}N & -0.1532925 & 0.4972252 & -0.2114070\end{array}$

$\begin{array}{llll}\text { C } & 0.8350031 & 1.3237120 & 0.4864251\end{array}$

$\begin{array}{llll}\text { C } & 2.1262977 & 0.5703329 & 0.8770376\end{array}$

$\begin{array}{llll}\text { O } & 2.9514878 & 1.0810749 & 1.6339012\end{array}$

$\begin{array}{llll}\text { C } & 0.2317379 & 2.0336121 & 1.7032682\end{array}$

H $\quad-1.6721523 \quad-0.3443433 \quad-2.9291980$

H $\quad-2.1894172 \quad-0.6807089-1.2432468$

$\begin{array}{llll}\text { H } & -2.6683791 & 0.8330278 & -2.0611781\end{array}$

H $\quad 1.1506859 \quad 2.0733709 \quad-0.2589486$

$\begin{array}{llll}H & 1.0093333 & 2.6136293 & 2.2138784\end{array}$

$\begin{array}{llll}H & -0.5759802 & 2.7080001 & 1.3894721\end{array}$

H $\quad-0.1741294 \quad 1.3075339 \quad 2.4253439$

H $\quad 1.6311483 \quad-0.9320797 \quad-0.4098080$

$\begin{array}{llll}N & 2.3012168 & -0.6625102 & 0.3077921\end{array}$

C $\quad 3.5930601 \quad-1.35910720 .4159509$

C $\quad 3.7708568 \quad-2.3936471 \quad-0.7078489$

O $4.7768092 \quad-2.4192256 \quad-1.4070539$

C $\quad 3.7831743 \quad-2.0094174 \quad 1.7979309$

$\begin{array}{llll}H & 4.3929224 & -0.6202898 & 0.2663661\end{array}$

URL: http://mc.manuscriptcentral.com/tandf/tmph 
H $\quad 4.7582001 \quad-2.5140905 \quad 1.8461581$

$\begin{array}{llll}\text { H } & 3.7524435 & -1.2309259 & 2.5699467\end{array}$

$\begin{array}{llll}H & 2.9942150 & -2.7459844 & 2.0106728\end{array}$

H $\quad 2.0708731 \quad-3.3635589 \quad-0.0808724$

$\begin{array}{llll}N & 2.7285183 & -3.2972024 & -0.8580411\end{array}$

C $\quad 2.8919019 \quad-4.5149742 \quad-1.6671273$

C $\quad 1.8199460-4.6737204 \quad-2.7714605$

$\begin{array}{llll}\text { O } & 1.6536886 & -5.7503863 & -3.3444868\end{array}$

C $\quad 2.9637108 \quad-5.7710448 \quad-0.7946527$

H $\quad 3.8503666 \quad-4.3767379-2.1940940$

$\begin{array}{llll}\text { H } & 3.0454878 & -6.6573026 & -1.4346774\end{array}$

$\begin{array}{llll}H & 3.8351909 & -5.7240078 & -0.1282801\end{array}$

H $\quad 2.0545032 \quad-5.8837677 \quad-0.1832286$

$\begin{array}{llll}H & 1.3207904 & -2.7068747 & -2.5343001\end{array}$

$\begin{array}{llll}N & 1.1260131 & -3.5406179 & -3.0837727\end{array}$

$\begin{array}{llll}\text { C } & 0.0703038 & -3.5090196 & -4.0983918\end{array}$

C $\quad 0.0687065 \quad-2.1310410 \quad-4.8073730$

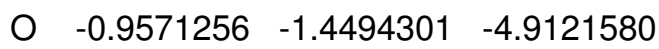

C $\quad-1.3040347 \quad-3.8524465 \quad-3.5232059$

H $\quad 0.3478475 \quad-4.2785249 \quad-4.8380595$

H $\quad-2.0667795 \quad-3.8256912-4.3116260$

H $\quad-1.2747678 \quad-4.8592363 \quad-3.0865012$

H $\quad-1.5992763 \quad-3.1298349-2.7501844$

H $\quad 2.0843882 \quad-2.3666941 \quad-5.2885405$

N $\quad 1.2744435 \quad-1.7407302 \quad-5.2958974$

C $1.4595059 \quad-0.5220795 \quad-6.0717129$

C $\quad 2.6441301 \quad-0.7914453 \quad-7.0248753$

$\begin{array}{llll}O & 3.4413066 & -1.7099068 & -6.8136647\end{array}$

URL: http://mc.manuscriptcentral.com/tandf/tmph 


$$
\begin{array}{llll}
\mathrm{C} & 1.7365610 & 0.7024881 & -5.1780155 \\
\mathrm{H} & 2.0391566 & 0.7889611 & -8.1806001 \\
\mathrm{~N} & 2.7494898 & 0.0710105 & -8.0738173 \\
\mathrm{C} & 3.8488613 & 0.0275524 & -9.0286965 \\
\mathrm{H} & 0.5356948 & -0.3472868 & -6.6474843 \\
\mathrm{H} & 1.8358314 & 1.6173242 & -5.7804419 \\
\mathrm{H} & 0.9126422 & 0.8546859 & -4.4686694 \\
\mathrm{H} & 2.6693302 & 0.5604624 & -4.6144159 \\
\mathrm{H} & 3.4771353 & -0.1394299 & -10.0501367 \\
\mathrm{H} & 4.4310723 & 0.9606611 & -9.0052481 \\
\mathrm{H} & 4.4986555 & -0.8072828 & -8.7438783
\end{array}
$$

Octapeptide (extended)

$$
\text { C } \quad-1.6015296 \quad-1.8805714 \quad 2.3774044
$$$$
\text { C } \quad-1.5896908 \quad-0.7306424 \quad 1.3785058
$$

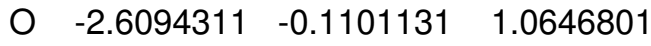$$
\begin{array}{llll}
H & 0.4556586 & -1.0288983 & 1.0302893
\end{array}
$$$$
\begin{array}{llll}
N & -0.3629157 & -0.4451823 & 0.8465530
\end{array}
$$$$
\text { C } \quad-0.1794910 \quad 0.5369091 \quad-0.2084299
$$$$
\text { C } \quad 1.1185258 \quad 0.1575073 \quad-0.9505692
$$$$
\begin{array}{llll}
O & 1.9276758 & -0.6496631 & -0.4719438
\end{array}
$$$$
\begin{array}{llll}
\text { C } & -0.1122078 & 1.9732887 & 0.3469699
\end{array}
$$$$
\begin{array}{llll}
H & -2.4978417 & -1.7947855 & 3.0002755
\end{array}
$$$$
\text { H } \quad-1.6439709-2.84053521 .8406806
$$$$
\text { H } \quad-0.7068681 \quad-1.8864723 \quad 3.0156836
$$$$
\text { H } \quad-1.0368833 \quad 0.4670951 \quad-0.8989338
$$$$
\begin{array}{llll}
H & -0.0087441 & 2.7105548 & -0.4620455
\end{array}
$$$$
\begin{array}{llll}
H & -1.0380597 & 2.1876806 & 0.8958275
\end{array}
$$ 
$\begin{array}{llll}H & 0.7423157 & 2.0803974 & 1.0299777\end{array}$

$\begin{array}{llll}H & 0.6506303 & 1.4733871 & -2.5038881\end{array}$

N $\quad 1.3119669 \quad 0.7847594 \quad-2.1358599$

$\begin{array}{llll}\text { C } & 2.5276427 & 0.6390056 & -2.9237133\end{array}$

C $\quad 2.5803622 \quad 1.8540920-3.8747794$

O $\quad 1.5727058 \quad 2.5395584 \quad-4.0979809$

C $\quad 2.5523364 \quad-0.6834855 \quad-3.7136442$

$\begin{array}{llll}\text { H } & 3.3904430 & 0.6609613 & -2.2378115\end{array}$

H $\quad 3.4887864 \quad-0.7862934 \quad-4.2799114$

H $\quad 2.4747221 \quad-1.5255520 \quad-3.0143948$

H $\quad 1.7099749-0.7244231 \quad-4.4187203$

H $\quad 4.5947864 \quad 1.4836750 \quad-4.2814716$

$\begin{array}{llll}N & 3.7822829 & 2.0825615 & -4.4532108\end{array}$

C $\quad 4.0017993 \quad 3.0977434 \quad-5.4750496$

$\begin{array}{llll}\text { C } & 5.3102714 & 2.7037495 & -6.1961988\end{array}$

$\begin{array}{llll}\text { O } & 6.0905495 & 1.8786016 & -5.6991653\end{array}$

C $\quad 4.0973451 \quad 4.5142943 \quad-4.8773453$

H $3.1590616 \quad 3.0665025 \quad-6.1853032$

H $\quad 4.2357462 \quad 5.2659153 \quad-5.6670283$

$\begin{array}{llll}\text { H } & 3.1694611 & 4.7438782 & -4.3386000\end{array}$

H $4.9439464 \quad 4.5806466 \quad-4.1796563$

H $\quad 4.9024601 \quad 4.0383758 \quad-7.7515172$

$\begin{array}{llll}\text { N } & 5.5418904 & 3.3356261 & -7.3688287\end{array}$

$\begin{array}{llll}\text { C } & 6.7739321 & 3.1809251 & -8.1318552\end{array}$

C $\quad 6.8272164 \quad 4.3793928-9.1060793$

$\begin{array}{llll}\text { O } & 5.8233301 & 5.0736310 & -9.3219788\end{array}$

C $\quad 6.8269205 \quad 1.8408566 \quad-8.8890685$

H $\quad 7.6258549 \quad 3.2303409 \quad-7.4337131$

URL: http://mc.manuscriptcentral.com/tandf/tmph 

H $\quad 7.7727365 \quad 1.7372440 \quad-9.4389300$
$\begin{array}{llll}H & 6.7508317 & 1.0140720 & -8.1719565\end{array}$
H $\quad 5.9968311 \quad 1.7716675 \quad-9.6060509$
H $\quad 8.8297231 \quad 3.9797614 \quad-9.5463669$
$\begin{array}{llll}N & 8.0208922 & 4.5872271 & -9.7062894\end{array}$
C $\quad 8.2344116 \quad 5.5960364-10.7358215$
C $\quad 9.5282188 \quad 5.1865242-11.4736300$
O $\quad 10.3026473 \quad 4.3482072-10.9915355$
C $\quad 8.35048517 .0139539-10.1455658$
H $\quad 7.3820528 \quad 5.5678200-11.4344996$
H $\quad 8.4850147 \quad 7.7613994-10.9399236$
H $\quad 7.4320060 \quad 7.2549652-9.5958989$
H $\quad 9.2069816 \quad 7.0755026 \quad-9.4597313$
H $\quad 9.1279535 \quad 6.5496705-13.0041748$
N $\quad 9.7525629 \quad 5.8208360-12.6466286$
C $\quad 10.9785868 \quad 5.6640012-13.4178378$
C $11.0588900 \quad 6.8969748-14.3444767$
$\begin{array}{llll}\text { O } & 10.0769264 & 7.6332401 & -14.5210763\end{array}$
C $\quad 11.0003841 \quad 4.3498742-14.2203810$
H $\quad 11.8321066 \quad 5.6692899-12.7200418$
H $11.9440695 \quad 4.2406624-14.7729617$
H $\quad 10.9046496 \quad 3.5016692-13.5311966$
H $\quad 10.1694126 \quad 4.3231249-14.9393564$
H $\quad 13.0357480 \quad 6.4413992-14.8414239$
N $\quad 12.2537178 \quad 7.0944159-14.9452741$
C $\quad 12.4856634 \quad 8.1405319-15.9308424$
C $\quad 13.7511292 \quad 7.7163324-16.7111764$
O $\quad 14.4710101 \quad 6.7967250-16.3084263$

URL: http://mc.manuscriptcentral.com/tandf/tmph 
C $\quad 12.6550890 \quad 9.5246106-15.2767141$

H $\quad 13.3539844 \quad 9.1700411-18.0953314$

N $\quad 14.0068515 \quad 8.4372266-17.8345002$

C $\quad 15.1824831 \quad 8.2211709-18.6692389$

H $\quad 11.6200916 \quad 8.1724511-16.6143716$

H $12.790392210 .3102774-16.0338891$

H $\quad 11.7563733 \quad 9.7630731-14.6944967$

H $\quad 13.5287121 \quad 9.5317528-14.6105597$

H $\quad 15.7486604 \quad 7.3902307-18.2337197$

H $\quad 14.8913797 \quad 7.9584096-19.6962512$

H $\quad 15.8196219 \quad 9.1173292-18.6931048$

Octapeptide (alpha-helix)

C $\quad-2.8430480 \quad-2.3803383 \quad 3.9023715$

$\begin{array}{llll}\text { C } & -3.0538594 & -1.8363243 & 2.5035294\end{array}$

O

$\begin{array}{llll}\text { H } & -3.8569117 & -0.1003200 & 3.2723942\end{array}$

N $\quad-3.5583057 \quad-0.5598854 \quad 2.4154666$

$\begin{array}{llll}\text { C } & -4.0665677 & -0.0190382 & 1.1489268\end{array}$

$\begin{array}{llll}\text { C } & -2.9781513 & 0.1647379 & 0.0729983\end{array}$

O

C $\quad-4.7978553 \quad 1.3039449 \quad 1.3958417$

H $\quad-3.5787775 \quad-3.1779732 \quad 4.0794207$

H $\quad-1.8470415 \quad-2.8361068 \quad 3.9648552$

H $\quad-2.9494038 \quad-1.6242085 \quad 4.6915507$

H $\quad-4.7616677 \quad-0.7488252 \quad 0.7041859$

$\begin{array}{llll}\text { H } & -5.1666415 & 1.7006690 & 0.4423677\end{array}$

H $\quad-5.6604154 \quad 1.1541580 \quad 2.0611183$

URL: http://mc.manuscriptcentral.com/tandf/tmph 

H $\quad-4.1279579 \quad 2.0525977 \quad 1.8442321$
H $\quad-1.5187619 \quad 0.1861333 \quad 1.5068839$
$\begin{array}{llll}N & -1.6976340 & 0.2892916 & 0.5104867\end{array}$
$\begin{array}{llll}\text { C } & -0.5705616 & 0.4094077 & -0.4107890\end{array}$
C $\quad-0.4052511 \quad-0.8021026-1.3554030$
$\begin{array}{llll}O & 0.2573997 & -0.6656526 & -2.3925774\end{array}$
$\begin{array}{llll}\text { C } & 0.7260205 & 0.6646916 & 0.3631593\end{array}$
$\begin{array}{llll}H & -0.7663353 & 1.2529362 & -1.0892194\end{array}$
H $\quad 1.5610473 \quad 0.7455489 \quad-0.3427813$
$\begin{array}{llll}H & 0.6610323 & 1.6026213 & 0.9326422\end{array}$
H $\quad 0.9432099-0.1607753 \quad 1.0581392$
$\begin{array}{llll}H & -1.5759524 & -2.0382463 & -0.1599737\end{array}$
N $\quad-0.9962036 \quad-1.9723041 \quad-1.0035100$
C $\quad-0.9045453 \quad-3.1394802 \quad-1.8810718$
C $\quad-1.5550713 \quad-2.9226893 \quad-3.2641194$

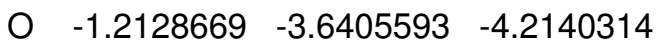
C $\quad-1.4960212 \quad-4.3777593 \quad-1.2012488$
H $\quad 0.1567165 \quad-3.3149260 \quad-2.1130279$
H $\quad-1.4248723 \quad-5.2318548 \quad-1.8855582$
H $\quad-0.9435179 \quad-4.6159463 \quad-0.2820716$
H $\quad-2.5517991 \quad-4.2218969 \quad-0.9387775$
$\begin{array}{llll}H & -2.7105766 & -1.3358022 & -2.5898847\end{array}$
N $\quad-2.4949418 \quad-1.9487594 \quad-3.3811631$
C $\quad-3.1223837 \quad-1.6775833-4.6749696$
C $\quad-2.1300869-1.1789715 \quad-5.7530320$
O
C $\quad-4.2808358-0.6891345 \quad-4.5172831$
H $\quad-3.4986878 \quad-2.6289977 \quad-5.0790550$

URL: http://mc.manuscriptcentral.com/tandf/tmph 
H $\quad-4.7222552 \quad-0.4925332-5.5021290$

H $\quad-5.0576208-1.1043722 \quad-3.8600946$

H $\quad-3.9355262 \quad 0.2621456 \quad-4.0886080$

H $\quad-0.7259529 \quad-0.6400848 \quad-4.3227596$

$\begin{array}{llll}N & -0.9638933 & -0.6267951 & -5.3186576\end{array}$

C $\quad 0.0485349 \quad-0.1458367 \quad-6.2551487$

C $\quad 0.9465330 \quad-1.2563850 \quad-6.8576035$

$\begin{array}{llll}\text { O } & 1.8287855 & -0.9534608 & -7.6635438\end{array}$

C $\quad 0.9010784 \quad 0.9546998 \quad-5.6196850$

H $\quad-0.4910589 \quad 0.2567826 \quad-7.1265873$

$\begin{array}{llll}H & 1.6847703 & 1.2537409 & -6.3257611\end{array}$

H $\quad 0.2805202 \quad 1.8291576 \quad-5.3803149$

$\begin{array}{llll}H & 1.3771904 & 0.6000622 & -4.6942200\end{array}$

H $\quad-0.0166890 \quad-2.7273855 \quad-5.7475537$

$\begin{array}{llll}N & 0.6675057 & -2.5417732 & -6.4865284\end{array}$

$\begin{array}{llll}\text { C } & 1.3989015 & -3.6755369 & -7.0373421\end{array}$

C $\quad 0.5984915 \quad-4.4755708 \quad-8.0878659$

$\begin{array}{llll}\text { O } & 1.1001815 & -5.4945528 & -8.5978532\end{array}$

C $\quad 1.9147072 \quad-4.6080238 \quad-5.9346112$

$\begin{array}{llll}H & 2.2485835 & -3.2437092 & -7.5896835\end{array}$

H $\quad 2.4145844 \quad-5.4718395 \quad-6.3891978$

H $\quad 2.6285832-4.0715466 \quad-5.2952709$

H $\quad 1.0850109 \quad-4.9676360 \quad-5.3093718$

H $\quad-0.9622809-3.1393469-8.0083769$

$\begin{array}{llll}N & -0.6273129 & -4.0102510 & -8.4252799\end{array}$

C $\quad-1.5129296-4.7137892 \quad-9.3649903$

C $\quad-1.1573292-4.3740252-10.8448140$

O

URL: http://mc.manuscriptcentral.com/tandf/tmph 

C $\quad-2.9707169 \quad-4.3989073 \quad-9.0537608$
H $\quad 0.6317408-5.2948489-10.4899651$
N $\quad 0.0644358 \quad-4.8470018-11.2203689$
C $\quad 0.6387883 \quad-4.5803014-12.5280619$
H $\quad-1.3120784 \quad-5.7884185-9.2228596$
H $\quad-3.6241623 \quad-4.9180967 \quad-9.7657089$
H $\quad-3.2191039 \quad-4.7242166 \quad-8.0341439$
H $\quad-3.1703845 \quad-3.3231642 \quad-9.1448066$
H $\quad-0.1334391 \quad-4.1065456-13.1456395$
H $\quad 1.5036959-3.9014689-12.4570813$
H $\quad 0.9638790 \quad-5.5150307 \quad-13.0090573$ 